\title{
ONWI-256
}

ONHII -256

DE82 010938

\section{Hydrological Model in STEALTH 2D Code}

\author{
Technical Report
}

October, 1979

\author{
Roger Hart \\ Ronald Hofmann
}

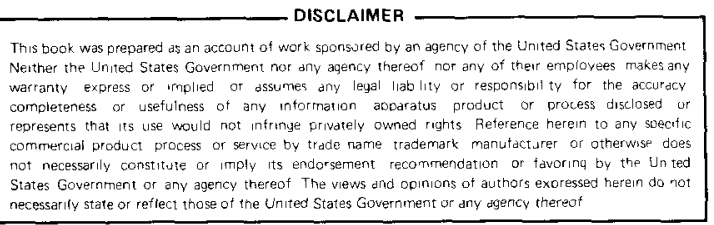

\section{Science Applications, Incorporated 2450 Washington Avenue San Leandro, CA 94577}

This report was prepared by Science Applications, Incorporated under Subcontract E512-01300 with Battelle Project Management Division, Office of Nuclear Waste Isolation under Contract No. DE-AC0676-RLO1830-ONWI with the U.S. Department of Energy. This contract was administered by the Battelle Office of Nuclear Waste Isolation. 


\section{DISCLAIMER}

This report was prepared as an account of work sponsored by an agency of the United States Government. Neither the United States Government nor any agency Thereof, nor any of their employees, makes any warranty, express or implied, or assumes any legal liability or responsibility for the accuracy, completeness, or usefulness of any information, apparatus, product, or process disclosed, or represents that its use would not infringe privately owned rights. Reference herein to any specific commercial product, process, or service by trade name, trademark, manufacturer, or otherwise does not necessarily constitute or imply its endorsement, recommendation, or favoring by the United States Government or any agency thereof. The views and opinions of authors expressed herein do not necessarily state or reflect those of the United States Government or any agency thereof. 


\section{DISCLAIMER}

Portions of this document may be illegible in electronic image products. Images are produced from the best available original document. 
.0

- 
Porous media fluid flow logic has been added to the twodimensional version of the STEALTH explicit finite-difference code. It is a first-order hydrological model based upon Darcy's Law. Anisotropic permeability can be prescribed through $x$ and $y$ directional permeabilities. The fluid flow equations are formulated for either two-dimensional translational symmetry or two-dimensional axial symmetry. The addition of the hydrological model to STEALTH is a first step toward analyzing a physical system's response to the coupling of thermal, mechanical, and fluid flow phenomena. 
- 
CONTENTS

$\underline{\text { Page }}$

SECTION 1 INTRODUCTION

SECTION 2 POROUS MEDIA FLUID-FLOW MODEL DESCRIPTION . . . . 3

2.1 BASIC EQUATIONS ........... 3

2.2 COMPARISON OF ·POROUS MEDIA FLUID-FLOW TO HEAT CONDUCTION . . . . . . . . . . 5

2.3 FORMULATION OF THE FLUID FLOW LOGIC FOR THE STEALTH 2D CODE . . . . . . . . 7

2.4 TWO-DIMENSIONAL FLUID FLOW MODEL CAPABILITIES . . . . . . . . . . 9

2.5 STABLE FLUID FLOW TIME STEPS . . . . . . 10

SECTION 3 VALIDATION OF THE FLUID-FLOW MODEL IN STEALTH 2D. 13

3.1 FLUID-FLOW MODEL TEST REQUIREMENTS . . . . 13

3.2 DESCRIPTION OF TEST PROBLEMS AND RESULTS FROM STEALTH 2D . . . . . . . . . . . 14

3.2.1 Test Type 1. Potential Boundary Condition Test . . . . . . . 15

3.2.2 Test Type 2. Fluid-Flow Boundary Condition Test . . . . . . 15

3.2.3 Test Type 3. Pressurized We11-Bore Simulation. . . . . . . . . 19

SECTION 4 DISCUSSION ............... 23

4.1 OVERVIEW OF COUPLING MECHANISMS . . . . . 23

4.2 EXPLICIT NUMERICAL TECHNIQUE FOR ANALYZING COUPLED RESPONSE ........... . . 25 
SECTION 4 Continued

4.3 STABILITY OF COUPLED PROBLEM

CALCULATIONS . . . . . . . . . 26

SECTION 5 RECOMENDATIONS . . . . . . . . . . 27

REFERENCES ....................... 29

APPENDIX A STEALTH FORM OF THE FLUID FLOW LOGIC . . . . A-1

APPENDIX B NEW AND REVISED STEALTH INPUT RECORDS . . . . B B-1

APPENDIX C ANALYTICAL SOLUTION OF FLUID FLOW PROBLEMS . . C -1 


\section{FIGURES}

Page

Figure 3-1. Fluid Flow Test Type 1 - Mesh Plots Showing Four Grid Orientations . . . . . . . 16

Figure 3-2. Fluid Flow Test Type 1 - Numerical

and Analytic Results for Several Grid

Orientations . . . . . . . . . . . . .

Figure 3-3. Fluid Flow Test Type 2 - Fluid Flow

Boundary Condition . . . . . . . . . . 18

Figure 3-4. Fluid Flow Test Type 3 - Pressurized We11bore Simulation 2-D Axial Symmetry Method . . . . . . . . . . . . . .

Figure 3-5. Fluid Flow Test Type 3 - Pressurized We1lbore Simulation 2-D Translational Symmetry Method . . . . . . . . . . . .

Figure 4-1. Fluid Flow-Heat Transfer-Mechanical Stress Coupling for a Saturated Porous Medium . . . . 24 


\section{SECTION 1}

INTRODUCTION

The principal mechanism for nuclide migration from a nuclear waste repository to the biosphere is for the nuclides to be transported by ground water that invades the vicinity of the repository. This vicinity is a region subjected to strong thermomechanical excitation, which, in some cases, could cause the permeability of the region to be modified significantly, resulting in more or less fluid flow. In order to analyze the fluid flow in this region, it is necessary to couple the fluid flow to the thermomechanical response.

Thermal and mechanical mechanisms have already been successfully coupled numerically and successfully used for the case of thermally induced creep in rock salt. Analyses have been performed in both two- and three-dimensions using the STEALTH* explicit finite-difference codes. Currently, STEALTH is being used to simulate hard rock situations in which joint slippage and fracture are the dominant thermally induced mechanisms. In the hard rock case, the fracture (natural and induced) pattern can give rise to pathways for fluid flow.

In order to analyze the time-dependent fluid flow, a coupled hydrological model must be introduced into existing thermomechanical analyses in order to analyse the importance of this mechanism. This report describes the successful coupling of a first-order hydrologic model (Darcy's Law) into the two-dimensional thermomechanical form of STEALTH.

* Solids and Thermal hydraulics codes for EPRI Adapted from Lagrange TOODY and HEMP", developed for Electric Power Research Institute by Science App̄lications, Inc. under EPRI Contract RP307. 
-

- 
SECTION 2

POROUS MEDIA FLUID-FLOW MODEL DESCRIPTION

\subsection{BASIC EQUATIONS}

Movement of fluid through a porous medium for most geomechanical applications is generally the result of a change in the fluid's pressure and/ or elevation. The equation of motion of the fluid is postulated by Darcy (Reference 1) as

$$
\mathrm{q}_{i}=-\mathrm{k}_{\Phi} \frac{\partial \Phi}{\partial \mathrm{x}_{i}}
$$

where $\mathrm{q}_{i}$ is the Darcy fluid flow velocity or specific discharge, $\mathrm{K}_{\Phi}$ is the hydraulic conductivity, $\Phi$ is the potential and the subscript $i$ denotes the fact that this is a vector equation.

The specific discharge, $q_{i}$, is the velocity of the fluid relative to the solid matrix through which it flows. It is actually a fictious flow velocity because it is an average value over a given cross section of the porous medium.

The potential or piezometric head, $\Phi$, represents the sum of the pressure energy and the potential energy per unit weight of fluid for each point in a saturated porous medium region. It is expressed by

$$
\Phi=\frac{p_{p}}{p_{f} g}+z
$$

where $p_{p}$ is the pore pressure, i.e., the pressure of the fluid in the pores of the medium, $p_{f}$ is the fluid density, $g$ is the gravity constant, 
and $z$ is the vertical coordinate, positive upward. $z$ represents the elevation above an arbitrary datum plane. $\frac{p_{p}}{\rho_{f} g}$ is generally called the pressure head
and $z$ the elevation head.

Hydraulic conductivity, $K_{\Phi}$, expresses the ease with which a fluid is transported through a porous matrix. It depends both upon the matrix and the fluid properties. The dependence of $\mathrm{K}_{\Phi}$ on these effects is generally expressed by (Reference 2)

$$
\mathrm{K}_{\Phi}=\frac{\rho_{\mathrm{f}} \mathrm{gk}}{\mu}
$$

where $\mathrm{k}$ is the intrinsic permeability and $\mu$ is the dynamic viscosity; $\rho_{f}$ and $g$ are the same as for Equation (2.2).

Both $\mathrm{K}_{\Phi}$ and $\mathrm{k}$ are second-order tensors and can therefore describe fluid-flow through an anisotropic medium. Hydraulic conductivity and intrinsic permeability have dimensions $[\mathrm{L}] / \mathrm{T}]$ and $[\mathrm{I}]^{2}$, respectively. The permeability is generally considered to be characteristic of the solid medium alone.

In addition to the fluid equation of motion, Equation (2.1), porous media fluid-flow must satisfy the basic principal of the conservation of mass. The equation for the conservation of fluid mass can be expressed as

$$
\frac{\partial}{\partial t}\left(n \rho_{f}\right)=-\frac{\partial}{\partial x_{i}}\left(\rho_{f} q_{i}\right)
$$

where $\mathrm{n}$ is the porosity of the medium, i.e., the ratio of the volume of the pores to the total volume of any representative element of the medium. The derivation of Equation (2.4) is widely available (for example, see References 2 or 3 ). 
The fluid mass storage equation is typically written by combining Equations (2.1) and (2.4),

$$
\frac{\partial}{\partial t}\left(n \rho_{f}\right)=\frac{\partial}{\partial x_{i}}\left(\rho_{f} k_{\Phi} \frac{\partial \Phi}{\partial x_{i}}\right) .
$$

Equation (2.5) contains the variables porosity, fluid density, hydraulic conductivity and potential, all of which can vary in time and space.

A discussion of the nonlinear behavior of porosity, hydraulic conductivity and fluid density is beyond the scope of the present study. Furthermore, to simplify the fluid flow model validation, the constitutive model for the fluid density-pressure relationship is assumed to be (Reference 3)

$$
\beta=\frac{1}{\rho_{f f}} \frac{d_{\rho_{f}}}{d p_{p}}
$$

where $\beta$ is the isothermal fluid compressibility, i.e., the measure of density change caused by changes in pressure under isothermal conditions. For an incompressible fluid $d_{p} / d_{p}=0$ or $\rho_{f}$ is constant. Porosity and hydraulic conductivity are considered constant for this study. Their behavior will be examined later as the research progresses.

\subsection{COMPARISON OF POROUS MEDIA FLUID-FLOW TO HEAT CONDUCTION}

A set of equations similar in form to those just developed for porous media fluid-flow arises from the analysis of heat conduction in a solid. (See Reference 4 for heat conduction development.) The relationship between the heat flux vector at a point and the change in temperature across the point in the direction of the vector can be expressed by Fourier's Law,

$$
\dot{\mathrm{h}}_{i}^{\prime \prime}=-\mathrm{K}_{\mathrm{T}} \frac{\partial \mathrm{T}}{\partial \mathrm{x}_{i}} \text {, }
$$


where $\ddot{h}_{i}^{\prime \prime}$ is the heat flux, $K_{T}$ is the thermal conductivity and $T$ is temperature. The differential equation for the conduction of heat in a solid is

$$
\rho_{s} c \frac{\partial T}{\partial t}=\frac{\partial}{\partial x_{i}}\left(K_{T} \frac{\partial T}{\partial x_{i}}\right)
$$

where $\rho_{s}$ is the density of the solid and $C$ is the specific heat capacity. Notice the similarities between Equations (2.1) and (2.7) and between Equations (2.5) and (2.8).

For constant thermal conductivity, Equation (2.8) becomes

$$
\frac{\partial T}{\partial t}=\frac{K_{T}}{\rho_{s} C} \frac{\partial^{2} T}{\partial x_{i}^{2}} .
$$

Solutions to this equation for various boundary conditions and geometries are given in Reference 4. The term $\frac{\mathrm{K}_{\mathrm{T}}}{{ }_{{ }_{\mathrm{S}} \mathrm{C}}}$ is called the thermal diffusivity, $\alpha_{T^{*}}$

Apply the following assumptions to the fluid mass storage equation, Equation (2.5);

- hydraulic conductivity, $\mathrm{K}_{\Phi}$, is constant,

- fluid density, $P_{f}$, is constant but not incompressible, $\beta \neq 0$,

- porosity, $n$, is constant,

- porous matrix is incompressible.

Using Equations (2.6) and (2.2), the storage equation becomes

$$
n p_{f}^{2} g \beta \frac{\partial \Phi}{\partial t}=\rho_{f} k_{\Phi} \frac{\partial^{2} \Phi}{\partial x_{i}^{2}}
$$


or

$$
\frac{\partial \Phi}{\partial t}=\frac{k_{\Phi}}{n \rho_{f} g \beta} \frac{\partial^{2} \Phi}{\partial x_{i}^{2}}
$$

which is precisely analogous to the heat conduction equation, Equation (2.9). The term $\frac{K_{\Phi}}{n p_{f} g \beta}$ is called the hydraulic diffusivity, $\alpha_{\Phi}$.

\subsection{FORMULATION OF THE FLUID FLOW LOGIC FOR THE STEALTH 2D CODE}

The fluid flow mode1 has been incorporated into the STEALTH 2D computer code (Reference 5) using analogous logic to that used for the heat conduction model already in STEALTH. For computational convenience, the relative fluid mass $M$ is introduced for use in the fluid mass storage equation. $M$ is defined as

$$
M \equiv \frac{\rho_{f}}{\rho_{f}^{o}}
$$

$P_{f}^{0}$ is an arbitrary reference value for fluid density.

The fluid mass storage equation, Equation (2.5), can be rewritten in terms of relative fluid mass as follows,

$$
\frac{\partial}{\partial t}(n M)=\frac{\partial}{\partial x_{i}}\left(M K_{\Phi} \frac{\partial \Phi}{\partial x_{i}}\right)
$$


Derivation of Equation (2.11a) is presented in more detail in Appendix A. The value of $\mathrm{MK}_{\Phi}$ represents an effective hydraulic conductivity, $\mathrm{K}_{e}$, for the porous medium domain. Thus, Equation (2.11a) can be rewritten, assuming constant porosity, as

$$
\frac{\partial M}{\partial t}=\frac{1}{n} \frac{\partial}{\partial x_{i}}\left(K_{e} \frac{\partial \Phi}{\partial x_{i}}\right)
$$

The two-dimensional finite-difference analog for Equation (2.11b) is

$$
\Delta M_{i-\frac{1}{2}, j-\frac{1}{2}}^{n+\frac{1}{2}}=\left(\dot{q}_{i-1}^{n+\frac{1}{2}}+\dot{q}_{i}^{n+\frac{1}{2}}+\dot{q}_{j-1}^{n+\frac{1}{2}}+\dot{q}_{j}^{n+\frac{1}{2}}\right) \frac{\Delta t^{n+\frac{1}{2}}}{q_{i-\frac{1}{2}, j-\frac{1}{2}}^{n+\frac{1}{2}} n_{i-\frac{1}{2}, j-\frac{1}{2}}}
$$

where $\Delta M_{i-\frac{1}{2}, j-\frac{1}{2}}^{n+\frac{1}{2}}$ is the change of relative fluid mass into (or out of) zone $\left(i-\frac{1}{2}, j-\frac{1}{2}\right)$ at time $n+\frac{1}{2},{ }^{\dagger} \Delta t^{n+\frac{1}{2}}$ is the time step over which the fluid mass change is evaluated, $\gamma_{i-\frac{1}{2}, j-\frac{1}{2}}^{n+\frac{1}{2}}$ is the true volume of the zone at time $n+\frac{1}{2}$ and $\dot{q}^{n+\frac{1}{2}}$ is the fluid flow across faces $i, i-1, j$, and $j-1$ of the zone. The fluid flow analog for a particular face is

$$
\dot{q}^{n+\frac{1}{2}}=K_{e}^{n+\frac{1}{2}} \nabla \Phi^{n+\frac{1}{2}}
$$

$\nabla \Phi$ is the gradient of potential evaluated at a particular face.

Equations (2.12) and (2.13) are solved exactly the same way as their heat conduction counterparts in the STEALTH 2D code. See Reference 5 for a complete description of the finite-difference logic.

The change in fluid mass is a contributing influence to the pore pressure change in the medium. The finite-difference analog describing

\footnotetext{
${ }^{\dagger}$ Subscript and superscript notation are defined in Reference 5.
} 
pore pressure change considering a compressible solid matrix, isothermal conditions, and constant porosity is

$$
\Delta p_{i-\frac{1}{2}, j-\frac{1}{2}}^{n+\frac{1}{2}}=\frac{2}{\beta_{i-\frac{1}{2}, j-\frac{1}{2}}^{n+\frac{1}{2}}}\left[\frac{M_{i-\frac{1}{2}, j-\frac{1}{2}}^{n+1}-M_{i-\frac{1}{2}, j-\frac{1}{2}}^{n}}{M_{i-\frac{1}{2}, j-\frac{1}{2}}^{n+1}+M_{i-\frac{1}{2}, j-\frac{1}{2}}^{n}}\right]
$$

Development of Equation (2.14) is discussed in Appendix A.

\subsection{TWO-DIMENSIONAL FLUID FLOW MODEL CAPABILITIES}

The calculations for change in fluid mass, fluid flow and pore pressure, Equations (2.12), (2.13) and (2.14), have been added to the STEALTH 2D code. Fluid density, pore pressure and potential are all calculated each timestep (cycle) for each zone in the model. The potential can include the elevation head or disregard the gravity effect, depending upon the nature of the problem.

The fluid flow logic is installed in STEALTH 2D so that pore water flow calculations can be performed independently of mechanical and thermal calculationsif desired. Mechanical and thermal coupling to the fluid flow model is also available. However, specific coupling mechanisms need to be developed before coupled tests can be performed. 
The fluid density, porosity and intrinsic permeability must be prescribed as initial conditions. The logic is designed to easily incorporate a more complicated fluid equation of state, porosity relationship and/or permeability constitutive assumptions as site-specific experimental data indicate.

Anisotropic permeability capability has been incorporated in to the model by allowing $x$ - and $y$-directional permeabilities. This capability, however, was not tested in the present study.

The following fluid flow boundary conditions have been made available in STEALTH 2D:

- potential (or pore pressure) history,

- zero head (or zero pore pressure),

- directional fluid flow history $\left(\dot{q}_{\mathbf{x}}\right.$ and $\left.\dot{q}_{\mathbf{y}}\right)$,

- impermeable boundary.

The fluid flow equations are formulated for either two-dimensional translational symmetry ( $x$ and $y$ are independent) or two-dimensional axial symmetry ( $x$ and $z$ are independent).

New and revised STEALTH 2D input records are shown in Appendix B.

\subsection{STABLE FLUID FLOW TIME STEPS}

For an incompressible porous media fluid flow problem, the stability criterion for the problem time step is controlled by the fluid diffusion. An estimation for the time step criterion can be obtained by using Equation (2.10).

$$
\frac{\partial \Phi}{\partial t}=\frac{\mathrm{K}_{\Phi}}{n \rho_{f} g \beta} \frac{\partial^{2} \Phi}{\partial x^{2}}
$$


The critical time step for this equation is found in like manner to that for the thermal critical time step. The minimum stable fluid flow time step is determined such that

$$
\Delta t^{n} \leq \frac{\left(\Delta l^{n}\right)^{2} n \beta \rho_{f} g}{2 k_{T}}
$$

where $\Delta l^{n}$ is the smallest fluid flow length across the zone. Reference 6 gives a derivation of the critical time step for equations of the form of Equation (2.10).

Equation (2.10) assumes the porous matrix is incompressible. If the matrix is allowed to compress, the stability requirement for the fluid mass storage equation must be altered to include the effect of the matrix compressibility. In addition, a mechanical stability criterion must now be considered when matrix compressibility exists. The critical time step formula for coupled mechanism analyses is discussed further in Section 4.3. 
-

- 
SECTION 3

VALIDATION OF THE FLUID-FLOW MODEL IN STEALTH 2D

\subsection{FLUID-FLOW MODEL TEST REQUIREMENTS}

A set of relatively simple, analytic test problems were analyzed to check the validity of the fluid flow model in the STEALTH 2D code. The following aspects of the fluid-flow calculations were checked:

- Are the potential gradient and direction of fluid-flow calculations correct for any model orientation?

- Is the calculation of total potential including the influence of gravity on the elevation head correct?

- What is the effect of fluid compressiblity on the calculation?

- Is the model properly formulated for problems with a divergent (i.e., cylindrical) symmetry geometry?

These questions were answered by comparing the numerical results from

STEALTH 2D to analytical solutions for selected transient fluid-flow problems. The analytic solutions were obtained by taking advantage of the similarity between fluid flow and heat conduction. Analytical solutions for various transient heat conduction problems are given in Reference 4 and the analogous fluid-flow solutions are included in Appendix C. These solutions are based upon the assumptions made for the form of the fluid mass storage equation given by Equation (2.10). Although other analytic solutions may be available which do not make these simplyfying assumptions, for the present study these solutions are sufficient for analyzing the above questions. 


\subsection{DESCRIPTION OF TEST PROBLEMS AND RESULTS FROM STEALTH 2D}

Three types of fluid-flow tests were performed.

Type 1. Potential boundary condition test.

Type 2. Fluid-flow boundary condition test.

Type 3. Pressurized we11-bore simulation (cylindrical symmetry) test.

A11 three types used the same material properties. These properties are described below.

$\begin{array}{lrl}\text { Fluid density } & \rho_{\mathrm{f}} & =1000 \mathrm{~kg} / \mathrm{m}^{3} \\ \text { Porosity } & \mathrm{n} & =1 \\ \text { Hydraulic conductivity } & \mathrm{K}_{\Phi} & =0.1 \mathrm{~m} / \mathrm{sec} \\ \text { Fluid bulk modulus } & \mathrm{K}_{\mathrm{f}}=10^{5} \mathrm{~N} / \mathrm{m}^{2},\left(\mathrm{~K}_{\mathrm{f}} \equiv 1 / \beta\right) .\end{array}$

The fluid bulk modulus was chosen so that the fluid is quite compressible (water's bulk modulus is $\sim 2 \times 10^{9} \mathrm{~N} / \mathrm{m}^{2}$ ). A highly compressible fluid was chosen to allow a larger time step for the fluid model calculation and to study the influence of the compressibility on the results.

The three test types were performed over transient time periods with types 1 and 3 being allowed to reach a steady state. Results for all the tests are presented as "snap-shot" plots of potential vs. distance across the body at selected times.

Although the two-dimensional code, STEALTH 2D, was used for these analyses, the test problems are all one-dimensional flow problems. The side boundaries were designated as impermeable in order to achieve the one-dimensional conditions. This kind of testing is severe in that the accuracy of the numerical model can be critically reviewed by observing the symmetric character of the computed results. 


\subsubsection{Test Type 1. Potential Boundary Condition Test. For this}

test, a constant potential differential of $50 \mathrm{~cm}$ was applied to a mesh in various orientations in order to test both $\mathrm{x}$ - and $\mathrm{y}$-components of the model. The orientations, illustrated in Figure 1, are:

Orientation No. 1 - Vertical flow with gravity acting downward. Orientation No. 2 - Flow and gravity vector rotated $30^{\circ}$ counterclockwise. orientation No. 3 - Flow and gravity vector rotated $25^{\circ}$ clockwise. orientation No. 4 - Horizontal flow with no gravity effects.

In each case, the steady state condition consistent with the constant potential differential at the boundaries, is to be computed.

Comparison of numerical and analytical results for the potential distribution is made in Figure 2 for the four cases. Agreement is quite good even though the grid is coarse. Better accuracy can be expected for a finer grid. Notice that for the first three grids, gravity provides an initial potential to the problem whereas for orientation No. 4 , only the pore pressure influences the potential calculation.

\subsubsection{Test Type 2. Fluid-Flow Boundary Condition Test. This test} involves the effects of fluid building up in a mesh. An inward fluid flow of $0.5 \mathrm{~m} / \mathrm{sec}$ was prescribed at one end of a grid while zero flow (impermeable boundary) was set at the other. The effect of gravity was not included. The mesh is shown in Figure $3 a$.

Snapshots of the potential as a function of time are compared on the graph in Figure $3 b$ for three selected times. The influence of the fluid compressibility can be seen by observing that the numerical results for potential fall below the analytical results as time progresses. The reason for the difference in potentials at later times is because fluid density is not constant as is assumed for the analytical solution but is inversely 


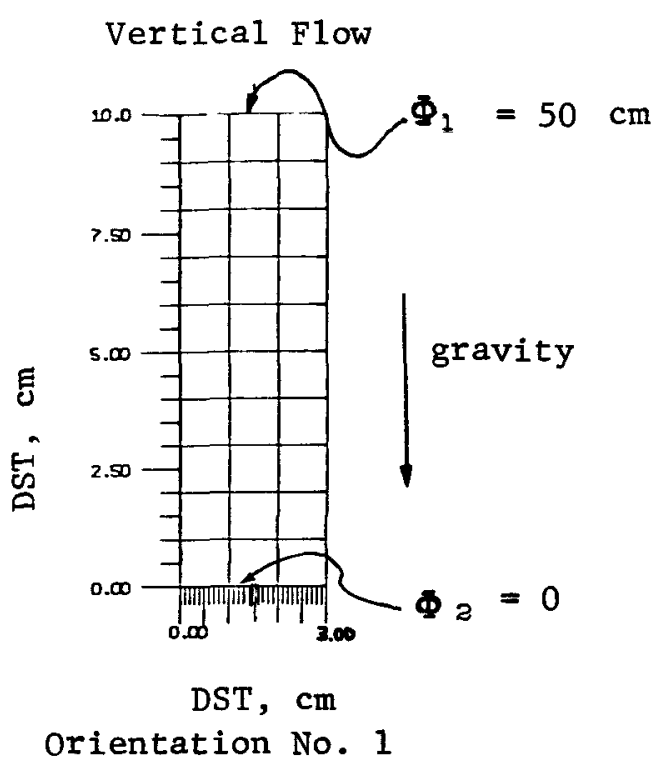

Flow Rotated $25^{\circ}$ Clockwise

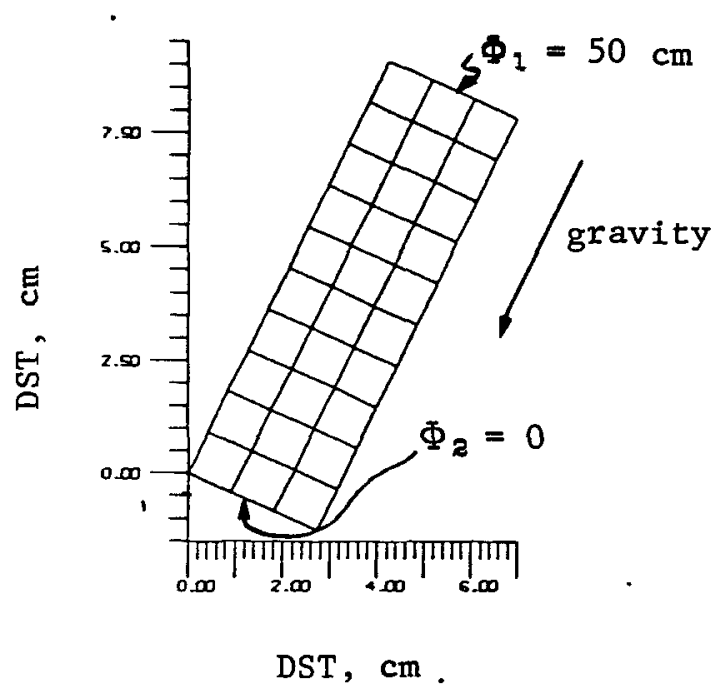

Orientation No. 3
Flow Rotated $30^{\circ}$ Counterclockwise

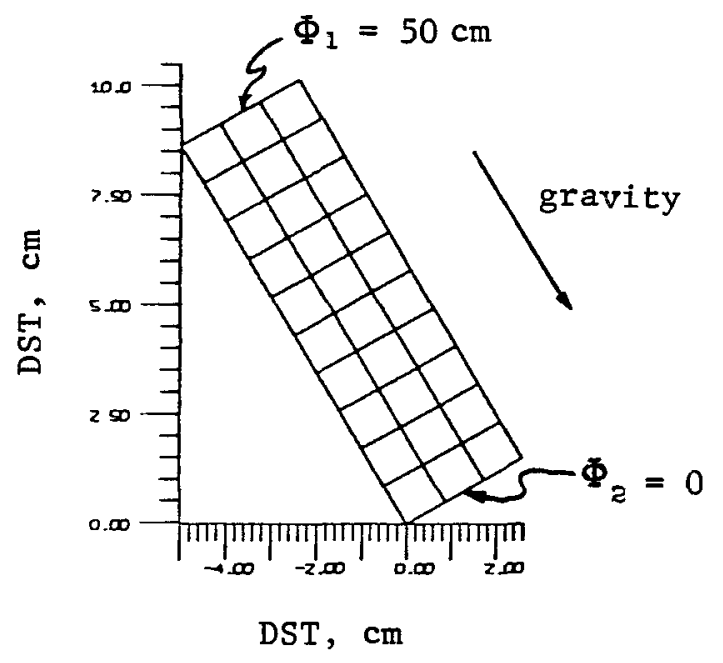

Orientation No. 2

Horizontal Flow

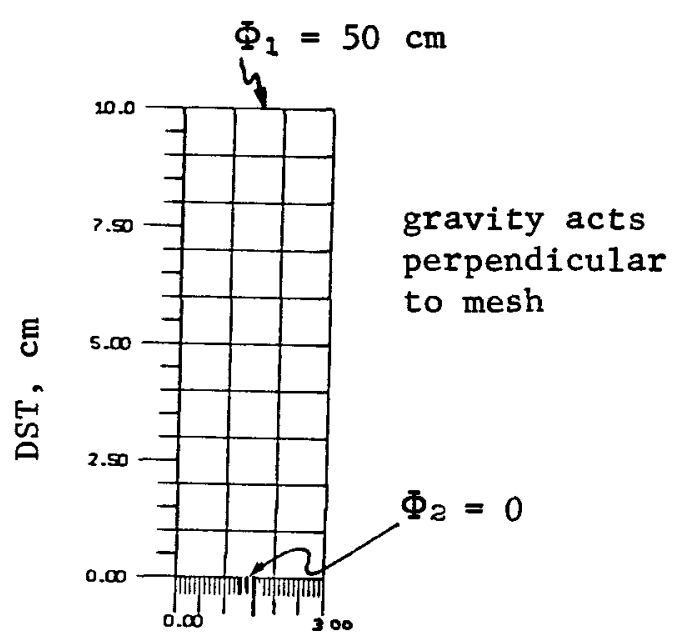

$$
\text { DST, cm }
$$

Orientation No. 4

Figure 3-1. Fluid Flow Test Type 1 - Mesh Plots Showing Four Grid orientations 
Vertical Flow

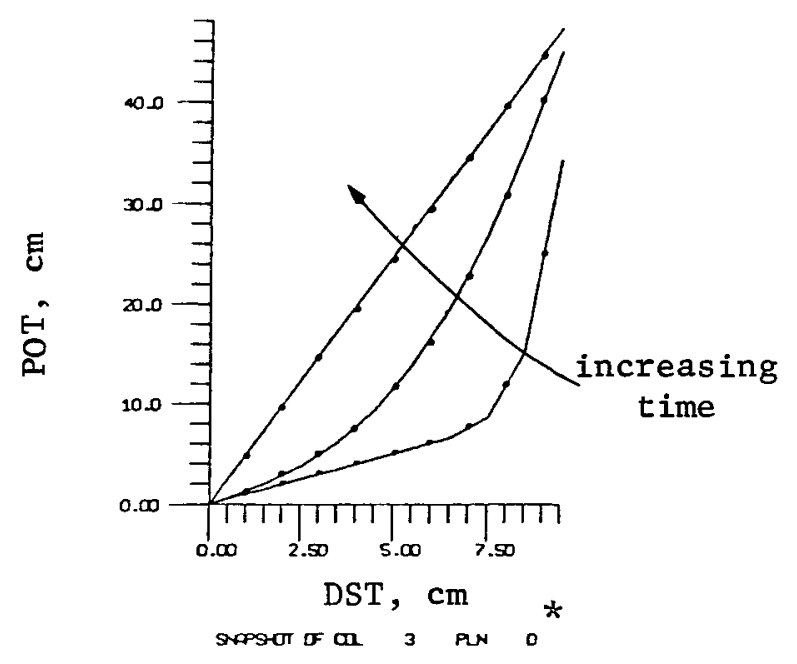

Orientation No. 1
Flow Rotated $30^{\circ}$ Counterclockwise

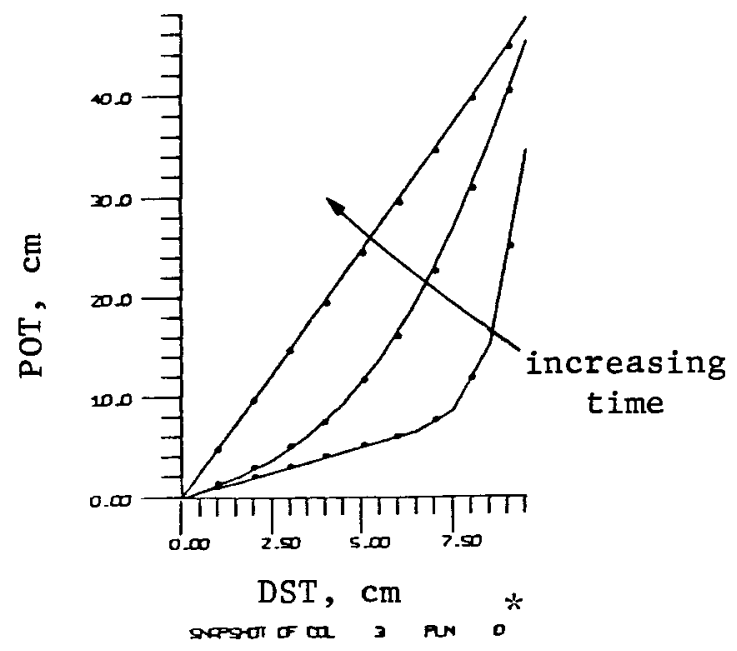

Orientation No. 2

- analytica1 solution

- numerical solution

Flow Rotated $25^{\circ}$ Clockwise

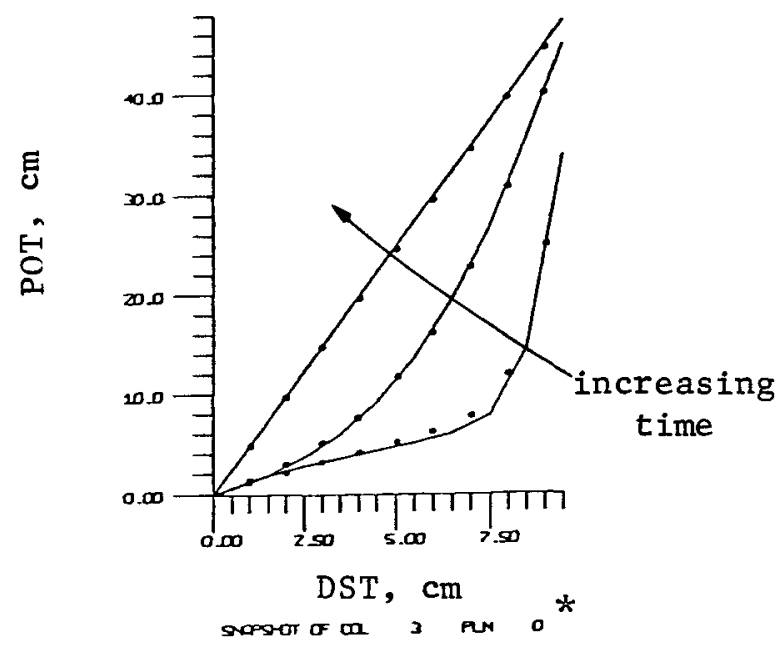

Orientation No. 3
Horizonta1 Flow

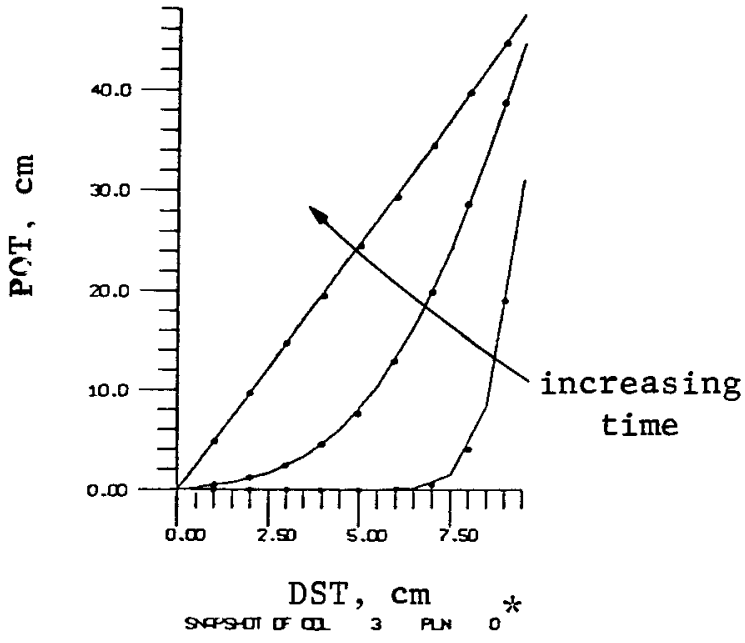

Orientation No. 4

*column 3 is the center vertical column of zones

Figure 3-2. Fluid Flow Test Type 1 - Numerical and Analytical Results for Several Grid orientations. 


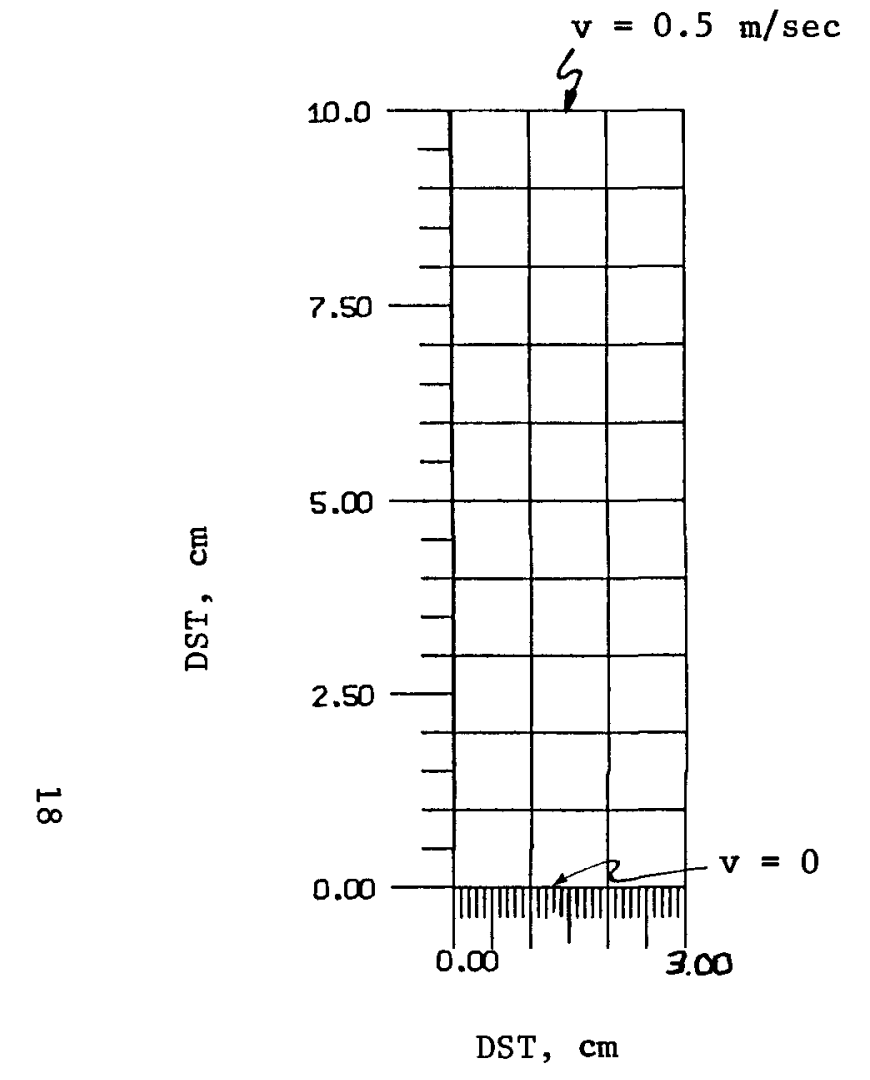

INITIA REPRESENTATIIN IF GRID

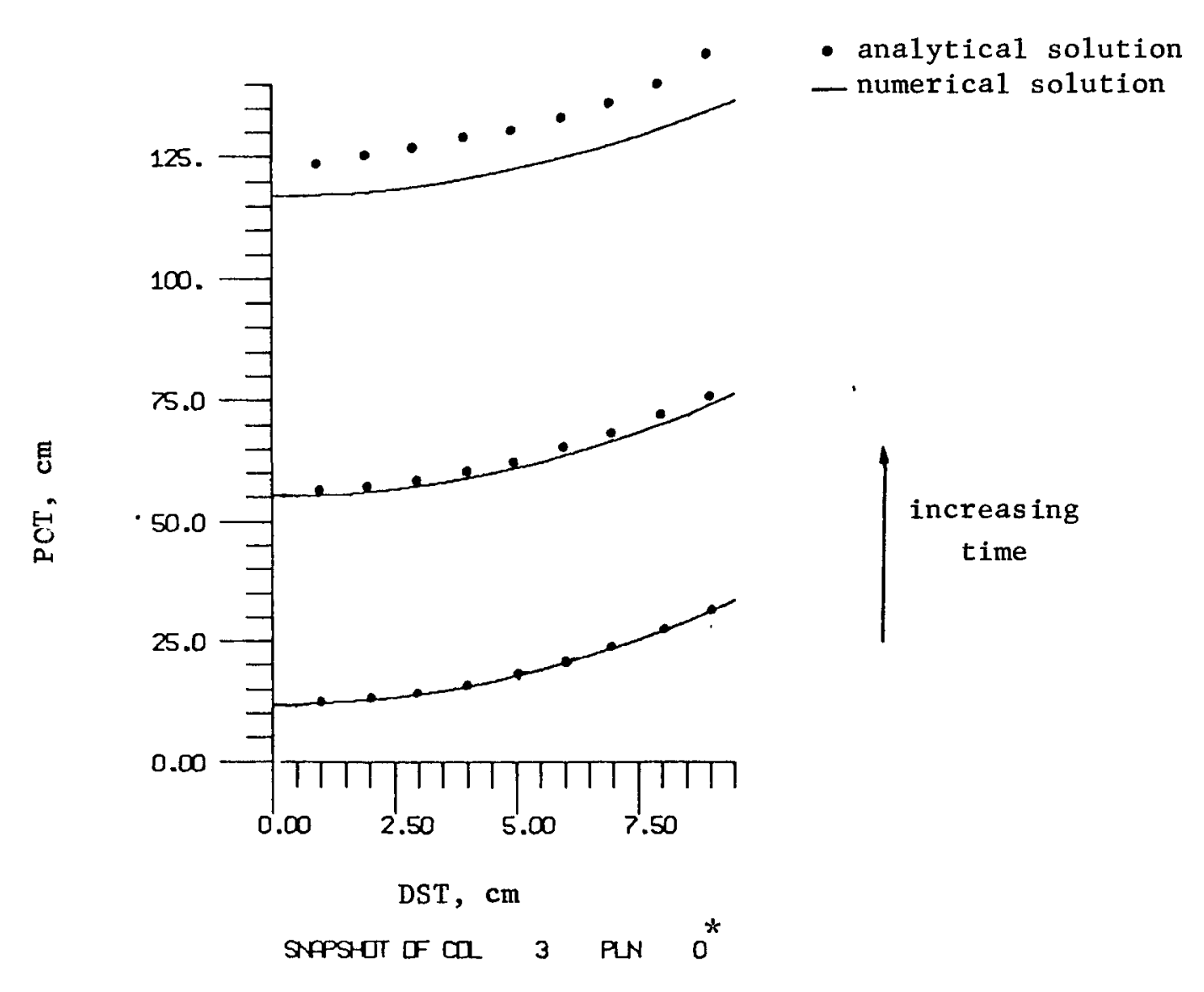

(b)

*column 3 is the center vertical column of zones

Figure 3-3. Fluid Flow Test Type 2 - Fluid Flow Boundary Condition 
related to the pore pressure by Equation (2.6). Thus, since the density increases with time, the pore pressure and potential are lower than they would be if the material was incompressible.

Since an analytical solution which included fluid compressibility was not not used for this test, the accuracy of the numerical results was not verified. However, the difference between the numerical potential values and the analytical values showed an inversely proportional correspondence to the increase in fluid density with time, as would be expected. In later research, further analysis of the fluid compressibility will be made and additional validation performed.

\subsubsection{Test Type 3. Pressurized We11-Bore Simulation. This test} case is typical of models used to simulate a constant pressure applied to the well-bore of a one-dimensional radial reservoir. For the case at hand, the pressure at the well-bore was set at $5000 \mathrm{~N} / \mathrm{m}^{2}$. Two approaches were examined for prescribing radial symmetry. They were:

1. describe the fluid mass storage equation in a two-dimensional axially symmetric form; and

2. define the grid using a two-dimensional translational symmetry geometry with a polar grid point description.

Approach 1 is illustrated by the grid in Figure $4 \mathrm{a}$, where the radial direction is horizonal and the axial direction vertical. The grid representation for Approach 2 is shown in Figure 5a. As can be seen by the accompanying potential plots on each figure, results for both methods are very close to the analytical solution. 


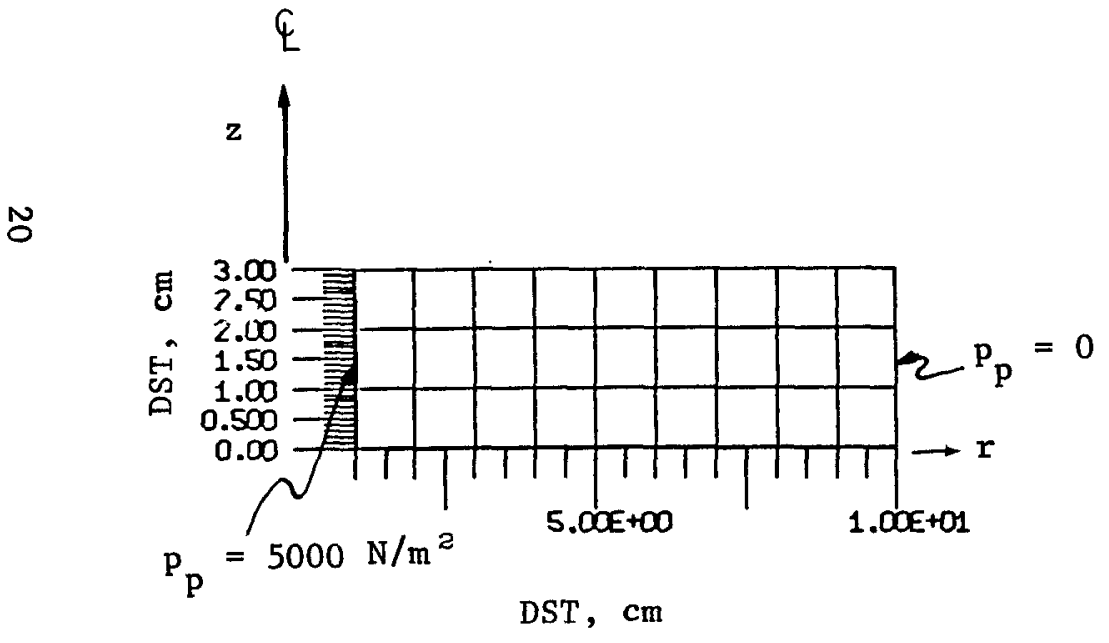

DITIAL REPRESENTATINN OF GRTD

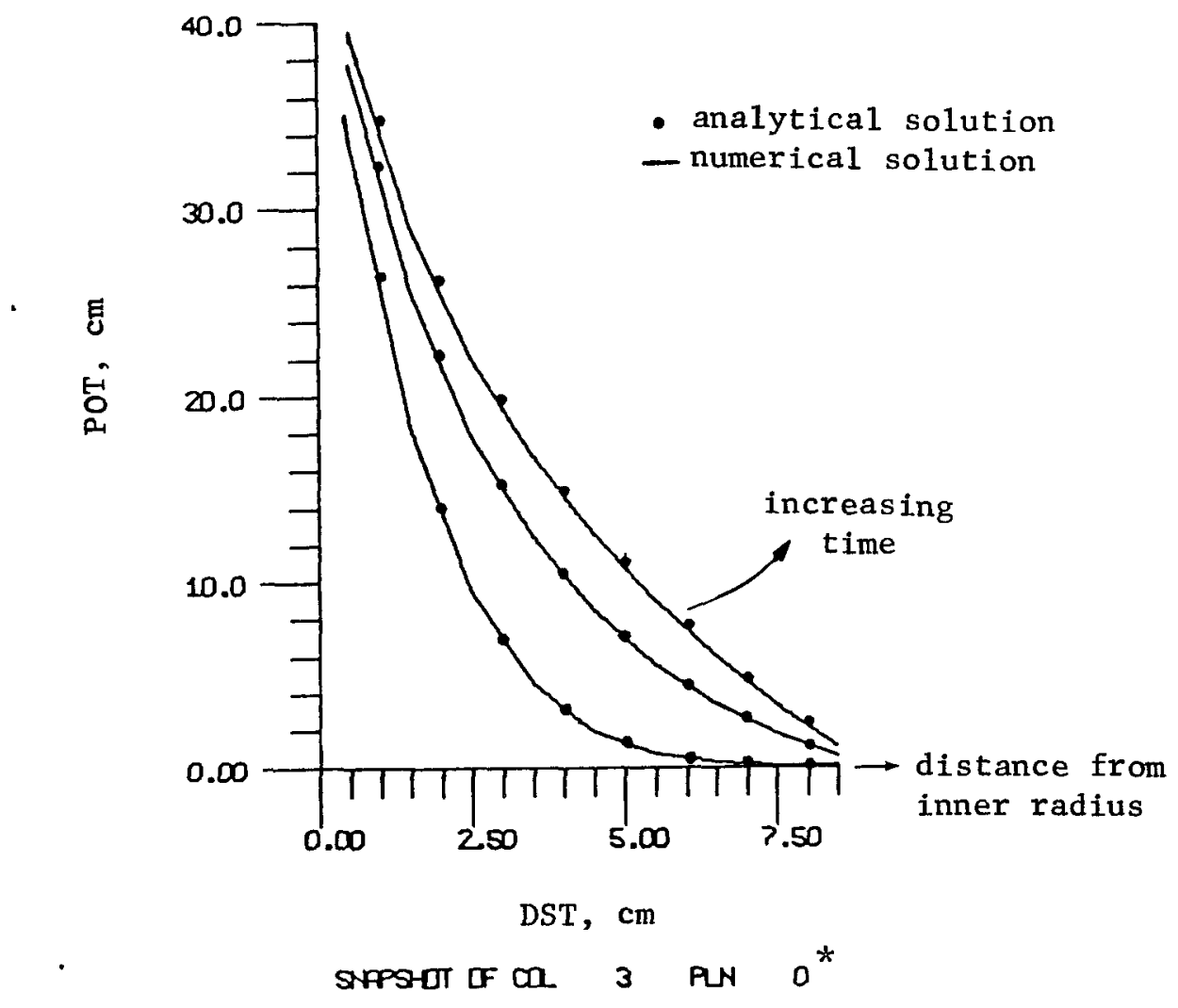

(b)

*column 3 is the center horizontal row of zones

Figure 3.4. Fluid Flow Test Type 3 - Pressurized Wellbore Simulation 2-D Axial Symmetry Method. 


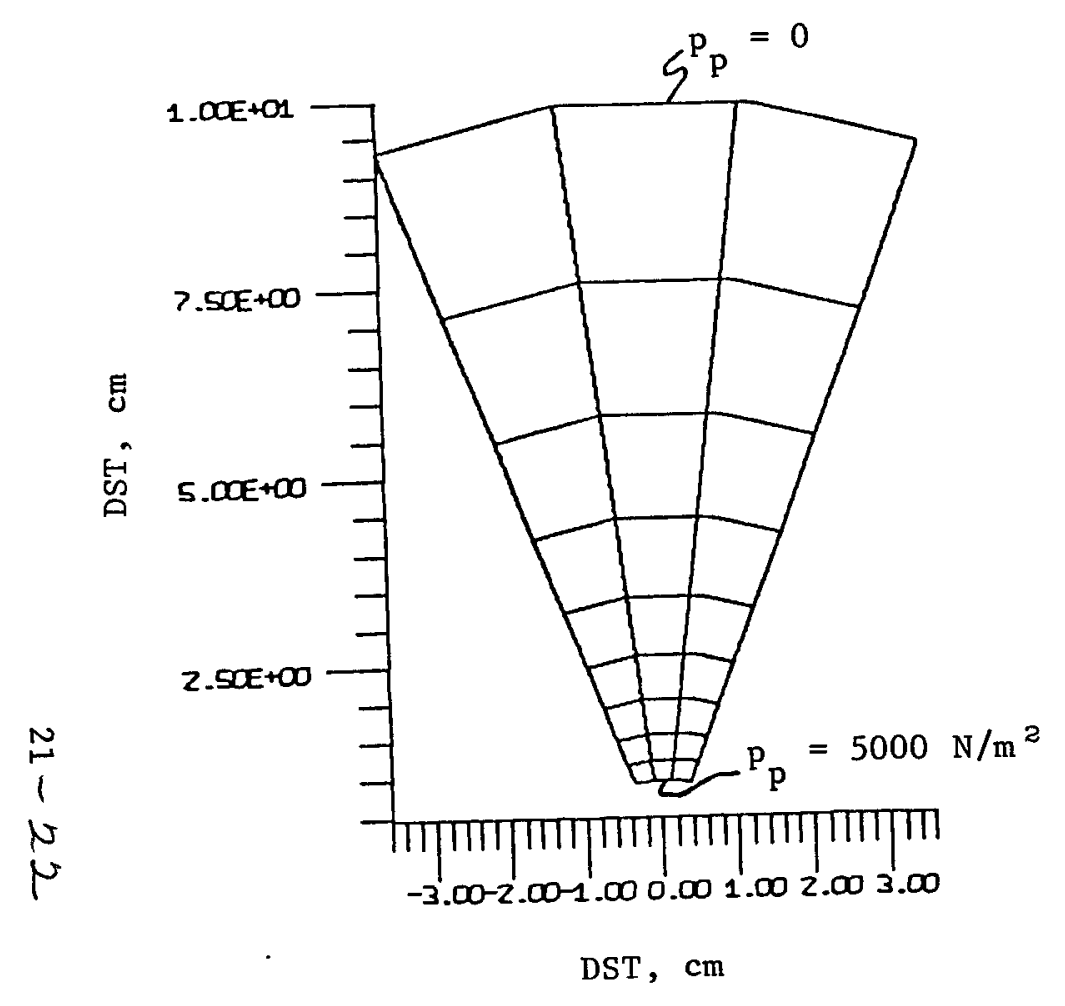

INTIIR REPRESENTIIIN IF GRII

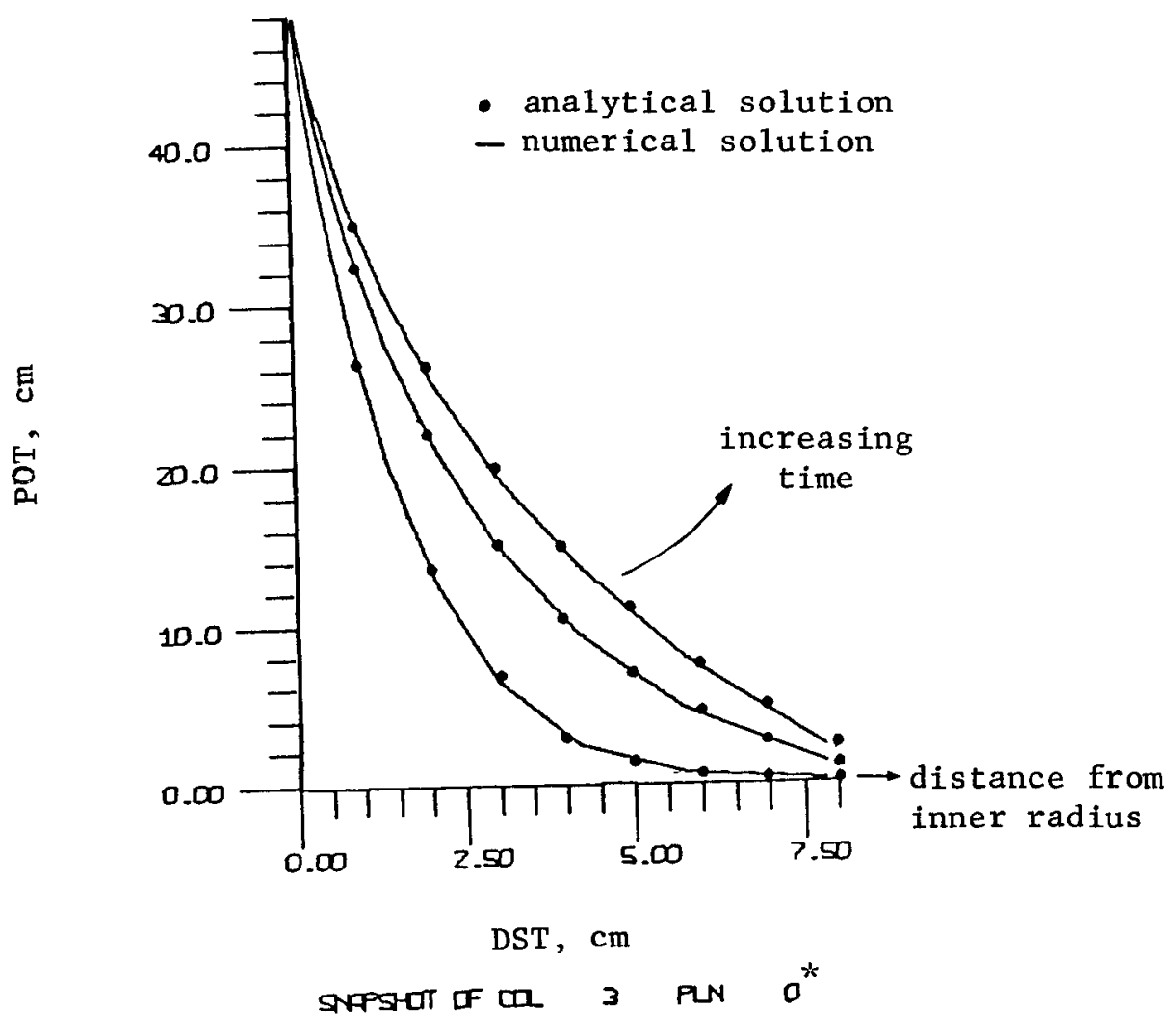

(b)

(a)

*column 3 is the center vertical column of zones

Figure 3-5. Fluid Flow Test Type 3 - Pressurized Wellbore Simulation 2-D Translational Symmetry Method. 
○

- 
SECTION 4

DISCUSSION

\subsection{OVERVIEW OF COUPLING MECHANISMS}

This report represents a first step toward analyzing a physical system's response to the coupling of thermal, mechanical and fluid flow phenomena. The coupling mechanism generally can be described by some constitutive relationship between any two of these physical processes. Figure 6 illustrates schematically the coupling mechanisms considered relevant for a geomechanical problem analysis.

As an example, examine the coupled mechanical stress fluid-flow behavior. Starting with a stress equilibrated porous medium, apply a perturbation through a change in pore pressure. This pressure differential can produce a total stress and total strain change for the system. A volumetric strain change for the porous medium can lead to a change in volume of the pores dependent upon the compressibility effect on the porosity. Likewise, the permeability may change. The changes in pore volume and permeability produce new changes in fluid flow and pore pressure. This cycle repeats until a new equilibrated state is reached which includes the pore pressure perturbation. The final response of the system may be completely different (and incorrect) if the phenomena are analyzed as being uncoupled, e.g., the mechanical stresses and strains might be assumed to have no effect on the fluid flow.

Coupling between fluid flow and heat transfer or mechanical stress and heat transfer can occur in a similar manner. However, as one may imagine, the complexity of the problem grows with each coupling effect so that an analytical solution to a completely coupled problem cannot be found. 


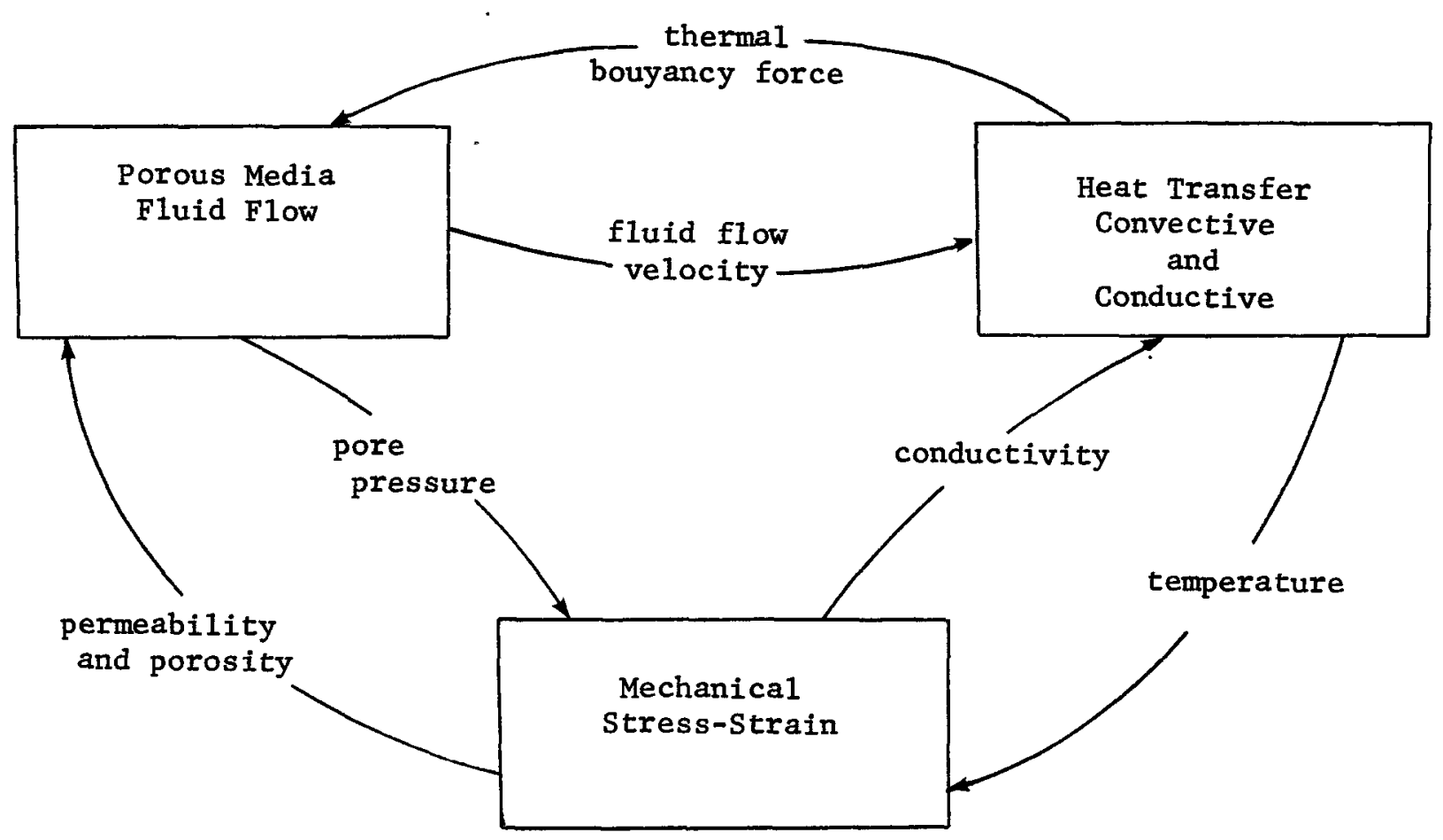

Figure 4-1. Fluid Flow-Heat Transfer-Mechanical Stress Coupling for a Saturated Porous Medium 


\subsection{EXPLICIT NUMERICAL TECHNIQUE FOR ANALYZING COUPLED RESPONSE}

A time-explicit, finite-difference numerical technique, such as that used in the STEALTH codes, does provide the capability for solving coupled problems. For this technique physical processes are considered coupled in time. That is, for each time step, the new-time system response is updated from old-time values through a calculational sequence that includes the coupling constitutive relationship.

The current version of STEALTH can solve coupled thermo-mechanical problems. Coupled effects from the simulation of a nuclear waste repository in a salt medium have been successfully modeled (Reference 7). Also, these studies included the physical effect of time-dependent salt creep behavior.

Inclusion of a porous media fluid-flow model into the STEALTH codes provides the basis for analyzing a completely coupled problem. The results of the present study indicate that the model is operating correctly. The next step is to incorporate a particular constitutive model to describe the coupling between the fluid flow and mechanical processes in an analogous way to that done for the thermo-mechanical coupling. Work on this step is already in progress. After that has been accomplished, a model prescribing the coupling between fluid flow and heat transfer can be added to provide a completely coupled system description. Since STEALTH can handle each material model separately, the inclusion of nonlinearities through the coupling mechanisms does not increase the difficulty of solving the problem. Constitutive models can be based on theory or laboratory experiments. 


\subsection{STABILITY OF COUPLED PROBLEM CALCULATIONS}

The stable time step for a completely coupled problem calculation is a function of mechanical wave propagation, fluid diffusion and heat transfer through the medium. If the problem has a transient mechanical shock, then a limiting criterion based upon the mechanical wave propagation is needed. If, on the other hand, the momentum effects can be neglected, the mechanical stability criterion is too restrictive. Methods are available (see Reference 8 ) to scale the mechanical time step for quasi-static problems so that the time scale is determined by another mechanism. These methods have been shown to be successful when applied to quasi-static thermomechanical problems where thermal diffusion determines stability (Reference 7).

The critical time step for a coupled stress fluid-flow problem is not as straightforward as that for a thermomechanical problem because of the effect of fluid and matrix compressibility, porosity and permeability on the stability criterion. In groundwater flow theory, the critical time step is written (from Reference 9)

$$
\Delta t \leq \frac{(\Delta l)^{2} \rho_{f} g[(1-n) \alpha+n \beta]}{4 K_{\Phi}}
$$

where $\alpha$ is the compressibility of the solid matrix. The form of this equation illustrates the effect of fluid density, porosity and permeability on the stability criterion. Nonlinearities in any of these variables could drastically reduce the critical time step.

It is clear that a better understanding of the stability condition for the fluid flow mechanism is required particularly in coupled analyses. Research in this area may be limited to parameter studies since a complete mathematical analysis could be cumbersome, if not impossible. 
SECTION 5

RECOMMENDATIONS

Inclusion of the fluid-flow model in STEALTH has been accomplished and the logical next step is to describe the coupled phenomena by including constitutive equations. The following steps are recommended as a systematic approach to this task.

1. Continue validation studies, using analytical solutions whenever possible, to analyze the nonlinear effects of anisotropic permeability, fluid compressibility and porosity on the fluid flow model.

2. Develop relationships for stress-dependent permeability and porosity for inclusion in the STEALTH code to describe the coupling between fluid flow and mechanical stress.

3. Analyze the time-step stability criterion for a coupled stressflow problem. This analysis may be performed analytically at first, assuming that material properties are constant. The effects of a nonlinear physical relationship will have to be determined through parameter studies.

4. Demonstrate the coupled stress-flow model by performance of test problems. Analytical solutions are axailable for problems which assume linear elastic material behavior and fluid flow governed by Darcy's Law.

5. Analyze coupled stress-flow problems where nonlinear material effects are included. Validation of the model for these problems will probably have to be based upon laboratory and field experimental results. 
6. Develop relationships for coupling mechanisms between fluid flow and heat transfer. These mechanisms should include the effect of natural convection.

7. Analyze the stability criterion for a completely coupled event. Parameter studies will be necessary.

8. Test the coupled stress-heat-fluid flow model with selected laboratory and field data. This should be most readily available from nuclear waste repository experimental studies.

Steps 1 through 4 may be accomplished in the near future since considerable information is already available on linearly coupled stressflow behavior. Steps 5 through 8 will require long-term studies since nonlinear effects are not well understood. Improvement of the model can only advance, after the constitutive mechanisms have been developed.

As the work on the porous media model progresses, the intention is that SAI's cracking and void strain model (CAVS) will be introduced into STEALTH 2D and the two models coupled. This will provide the capability of either describing the system as an equivalent porous medium or as a discretely fractured medium. 


\section{REFERENCES}

1. Darcy, H., 1865. Les Fontaines Publiques de la Ville de Dijon, V. Dalmont, Paris, France.

2. Davis, S. N., and R. J. M. De Wiest, 1966. Hydrogeology, John Wiley \& Sons, Inc., New York, NY.

3. Bear, J., 1972. Dynamics of Fluids in Porous Media, American Elsevier, Inc., New York, NY.

4. Carslaw, H. S., and J. C. Jaeger, 1959. Conduction of Heat in Solids, (2nd ed.) Oxford University Press, Oxford, England.

5. Hofmann, R., 1976. STEALTH, A Lagrange Explicit Finite-Difference Code for Solids, Structural, and Thermohydraulic Analysis, Vol. 1, User's Manual, EPRI NP-260, Science Applications, Inc., San Leandro, $\overline{C A}$, for Electric Power Research Institute, Palo Alto, CA.

6. Carnahan, B., H. A. Luther, and J. 0. Wilkes, 1969. Applied Numerical Methods, John Wiley \& Sons, Inc., New York, NY.

7. Wahi, K. K., D. E. Maxwe11, and R. Hofmann, 1978. A Two-Dimensional Simulation of the Thermomechanical Response of Project Salt Vault Including the Excavation Sequence, Y/OWI/SUB-78/76549/2, Science Applications, Inc., San Leandro, CA, for the Office of Waste Isolation, Union Carbide Corporation-Nuclear Division, Oak Ridge, TN.

8. Maxwe11, D. E., R. Hofmann, and K. K. Wahi, 1978. An Optimization Study of the Explicit Finite-Difference Method for Quasi-Static Thermomechanical SimuTations, Y/OWI/SUB-78/16549/3, Science Appl ications, Inc., San Leandro, CA, for the Office of Waste Isolation, Union Carbide Corporation-Nuclear Division, Oak Ridge, TN.

9. Rushton, K. R. and L. M. Toml inson, 1971. "Digital Computer Solutions of Groundwater Flow", Journal of Hydrology 12 (339-362). 
0

- 
APPENDIX A

STEALTH FORM OF THE FLUID FLOW LOGIC

A.1 FLUID MASS STORAGE EQUATION

The fluid mass storage equation written in Section 2.1 of this report is :

$\frac{\partial}{\partial t}\left(n p_{f}\right)=\frac{\partial}{\partial x_{i}}\left(p_{f} K_{\Phi} \frac{\partial \Phi}{\partial x_{i}}\right)$.

Equation (A.1) is introduced into the STEALTH code by using a dimensionless density called the relative fluid mass, M. The relative fluid mass may be defined for a constant unit volume in terms of the fluid density, i.e.,

$$
M \equiv \frac{\rho_{f}}{\rho_{f}^{o}},
$$

where the superscript o refers to an initial or a reference state. Dividing Equation (A.1) by the reference partial density, $\rho_{f}^{\circ}$, yields

$$
\frac{\partial}{\partial t}\left(\mathrm{n} \frac{\rho_{f}}{\rho_{f}^{o}}\right)=\frac{\partial}{\partial \mathrm{x}_{i}}\left(\frac{\rho_{f}}{\rho_{f}^{0}} K_{\Phi} \frac{\partial \Phi}{\partial \mathrm{x}_{i}}\right)
$$

which can be written, using Equation (A.2), as

$$
\frac{\partial}{\partial t}(n M)=\frac{\partial}{\partial x_{i}}\left(M K_{\Phi} \frac{\partial \Phi}{\partial x_{i}}\right)
$$

Equation (A.3) is the form of the fluid mass storage equation used in STEALTH. It was chosen because it is convenient and, depending on the choice of the reference state, can be written in a round-off free explicit finitedifference analog. 


\section{A.2 FLUID DENSITY - PORE PRESSURE RELATIONSHIP}

Under isothermal conditions, fluid density change may be assumed proportional to pore pressure change through the compressibility coefficient, $\beta$. The relationship is

$$
d p_{p}=\frac{1}{\beta} \frac{d \rho_{f}}{\rho_{f}} .
$$

The finite-difference analog for Equation (A.4) may be written, using STEALTH notation for time centering, as

$$
\Delta p_{p}^{n+\frac{1}{2}}=\frac{1}{\beta}\left(\frac{\rho_{f}^{n+1}-\rho_{f}^{n}}{\rho_{f}^{n+\frac{1}{2}}}\right)
$$

where $\rho_{f}^{n+\frac{1}{2}}$ is a time-averaged value $\left[\rho_{f}^{n+\frac{1}{2}}=\left(\rho_{f}^{n+1}+\rho_{f}^{n}\right) / 2\right]$. Equation (A.5) can be written using the definition for relative fluid mass, i.e.,

$$
\Delta p_{p}^{n+\frac{1}{2}}=\frac{2}{\beta}\left(\frac{M^{n+1}-M^{n}}{M^{n+1}+M^{n}}\right) \text {. }
$$




\section{APPENDIX B}

NEW AND REVISED STEALTH INPUT RECORDS

The input records and documentation shown in this Appendix are based on published data for STEALTH Version 3-2A.* Changes to existing input records are noted with a black vertical stripe in the right margin, and new input records are noted with the word "NEW" in the upper right corner. Revision information in the lower right corner has been omitted because the hydrological model is not yet a part of the standard STEALTH distribution tape.

*Hofmann, R., 1976. "STEALTH, A Lagrange Explicit Finite-Difference Code for Solids, Structural, and Thermohydraulic Analysis. User's Manual", Science Applications, Inc., San Leandro, CA, for Electric Power Research Institute, Palo Alto, CA, EPRI NP-260, Vo1. 1, August. 


$$
\text { Page } 1 \text { of } 1
$$

The DTS input record specifies the time step stability criterion to be used for this problem. It is optional for both start and restart runs. When used, one and only one record is required; when not used, default values are assumed.
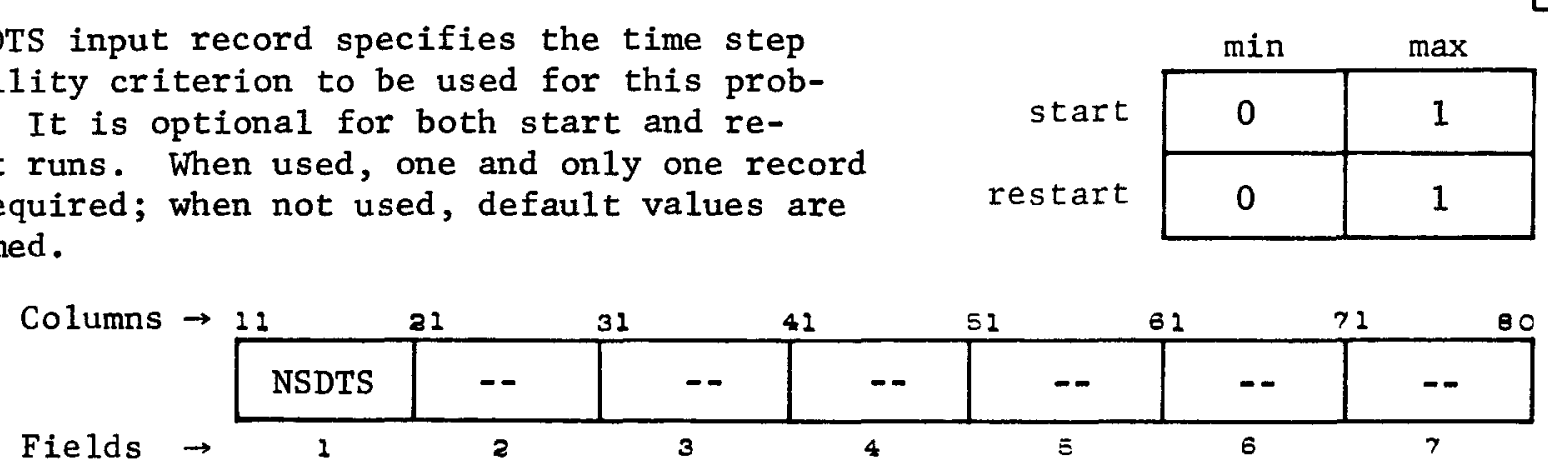

Input

Field Variable

Number Name Description of Input Variable

1 NSDTS Dynamic-thermal-static hydrology indicator switch. The value of NSDTS indicates the type of time step control to be used. The value of NSDTS must be integral and less than or equal to 7 . The following definitions apply:
$\leq 1.0$
$=2.0$
stress wave dynamic only (default)
$=3.0$
$=4.0$
$=5.0$
$=6.0$
$=7.0$
dynamic relaxation
thermal transient only
complete mechanical and thermal response
hydrology transient only
coupled mechanical stress-hydrology response
coupled thermal-mechanical-hydrology response

If the field containing NSDTS is left blank, then NSDTS is assumed to be equal to 1.0 , i.e., stress wave dynamic. If the value of NSDTS is greater than 7.0 , the message,

\section{DYNAMIC-THERMAL-STATIC-HYDROLOGY INDICATOR SWITCH IMPROPERLY DEFINED}

is written on the print and the message files and the program terminates immediately in subroutine PRBINP.

2-7 -- Fields 2-7 must be left blank or the FLDCHK message is written on the print and the message files and the program terminates at the end of the GENERATOR phase group. (See Appendix "Messages".) 


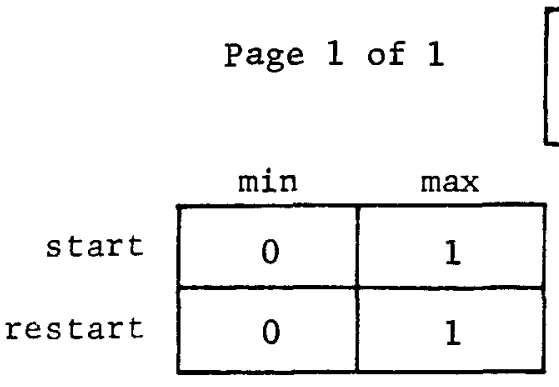

The GRV input record specifies values of gravity acceleration components for an entire problem.* If the GRV input record is omitted, the default values are used. The GRV record may be used at restart time to change the values of gravity acceleration components.

\begin{tabular}{|c|c|c|c|c|c|c|c|}
\hline Columns $\rightarrow$ & & 21 & 31 & 41 & 51 & 61 & 72 \\
\hline & GRVX & GRVY & GRVZ & -- & -- & -- & -- \\
\hline
\end{tabular}

Input

Field Variable

Number Name

Description of Input Variable

$1 \quad$ GRVX

Acceleration of gravity in the $x$-direction. A positive value of GRVX results in an acceleration in the positive $x$-direction, while a negative value of GRVX results in an acceleration in the negative $\mathrm{x}$-direction. The default value of GRVX is 0.0 .

$2 \quad$ GRVY

Acceleration of gravity in the y-direction. A positive value of GRVY results in an acceleration in the positive y-direction, while a negative value of GRVY results in an acceleration in the negative $y$-direction. The default value of GRVY is 0.0 . GRVY must be left blank for one-dimensional problems.

3 GRVZ Acceleration of gravity in the z-direction. A positive value of GRVZ results in an acceleration in the positive z-direction, while a negative value of GRVZ results in an acceleration in the negative z-direction. The default value of GRVZ is 0.0 . GRVZ must be left blank for one- and two-dimensional problems.

4-7 -- Fields 4-7 must be left blank or the FLDCHK message is written on the print and the message files and the program terminates at the end of the GENERATOR phase group. (See Appendix "Messages".)

${ }^{*}$ For hydrological type problems (NSDTS $=5,6$ or 7 ), if gravity effects are present, GRVX and GRVY should be used and GRVZ must equal 0 . If no gravity effect is present, GRVZ must be set to a value consistent with units of the problem and GRVX and GRVY set equal to zero. 


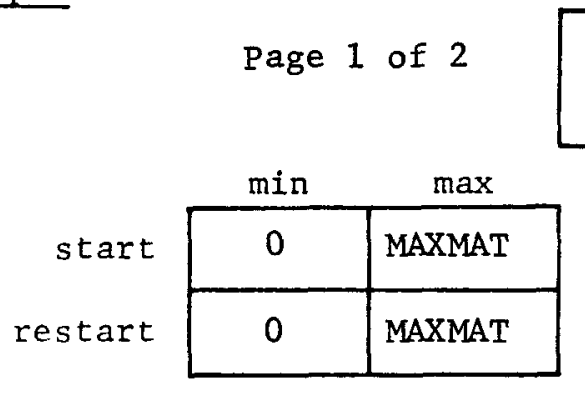

The 111 input record specifies the type of Equation-of-state model for material MPN. If the 111 input record is omitted, the default value is used. The 111 input record may be used at restart time to change the equation-of-state model type.

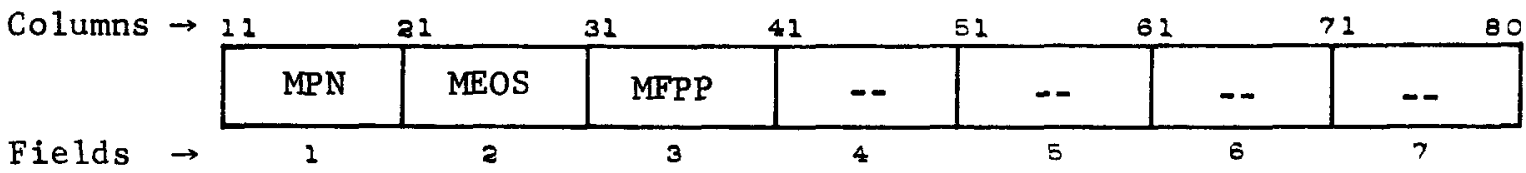

Input

Field Variable

Number Name

Description of Input Variable

1 MPN

2 MEOS Equation-of-state model type. The value of MEOS uniquely indenti-

Material property number. The value of MPN uniquely identifies a material model. A model consists of an equation of state and strength, explosive, and thermal descriptions. A single value of MPN links together a set of MAT phase input records for one material model. The value of MPN must be integral and must be greater than or equal to 1 and less than or equal to MAXMAT. (See Appendix "Maximum".) If MPN is out of this range, a RGEERR message is written on the print and the message files and the program terminates immediately in subroutine MATINP. (See Appendix "Messages".) fies the type of equation-of-state model for material MPN. The equation of state defines a relationship for pressure as a function of relative volume and internal energy density. The value of MEOS must be integral and must be greater than or equal to 1 and less than or equal to MAXEOS. (See Appendix "Maximums".) If MEOS is out of this range, a RGEERR is written on the print and the message files and the program terminates immediate in subroutine MATINP. (See Appendix "Messages".) The following definitions apply:

$$
\begin{aligned}
& =1.0 \text { function of relative volume only (default) } \\
& =2.0 \text { function of internal energy density only } \\
& \text { =3.0 function of relative volume and internal energy } \\
& =4.0 \quad \mathrm{p}-\alpha \text { compaction model (not yet programmed) } \\
& =5.0 * \quad \text { JWL chemical explosive model (not yet programmed) } \\
& =6.0^{*} \text { incremental function of volumetric strain and } \\
& =7.0-\quad \text { user-supplied mode1s } \\
& 10.0
\end{aligned}
$$

A description of each model is presented in Appendix "Material Models". Coefficients for a particular model are entered on input records $121,122,123$.

${ }^{\star}$ See next page.

Rev . 


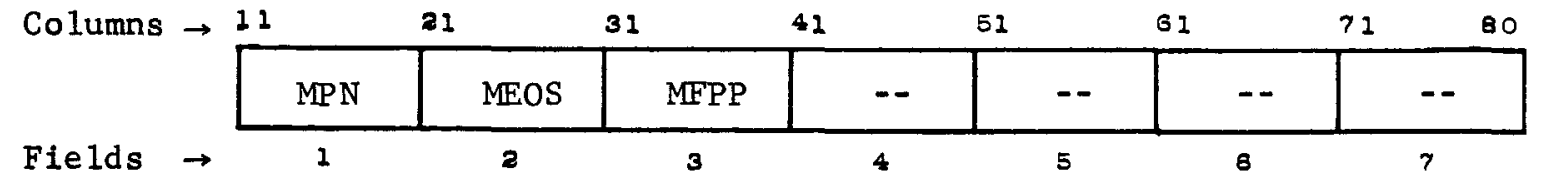

Input

Field Variable Number Name Description of Input Variable

$3 \quad$ MFPP

Fluid pore pressure model type. The value of MFPP uniquely identifies the type of fluid pore pressure model for material MPN. The equation of state defines a relationship for pore pressure as a function of relative fluid mass and relative zone volume. The value of MFPP must be integral and must be greater than or equal to 1 and less than or equal to MAXEOS. (See Appendix "Maximums".) If MFPP is out of this range, a RGEERR is written on the print and the message files and the program terminates immediately in subroutines MATINP. (See Appendix "Messages".) The following definitions apply:

$$
\begin{aligned}
& =1.0 \quad \text { incrememental function of relative fluid mass and } \\
& \text { temperature }
\end{aligned}
$$

A description of each model is presented in Appendix "Material Models". Coefficients for a particular model are entered on input records 124 and 125 .

4-7 -- Fields 4-7 must be left blank or the FLDCHK message is written on the print and the message files and the program terminates at the end of the GENERATOR phase group. (See Appendix "Messages".)

\footnotetext{
*Use MEOS $=6$ for incremental thermal-mechanical or hydraulic-thermal-mechanical analyses. The equation of state parameters on the 122 input record are:

EOS2 = matrix bulk modulus

EOS3 = solid grain bulk modulus

EOS4 = matrix volumetric thermal expansion coefficient
} 
The 116 input record specifies the type of matrix permeability model for material MPN. If the 116 input record is omitted, default values are used. The 116 input record may be used at restart time to change matrix permeability model types.
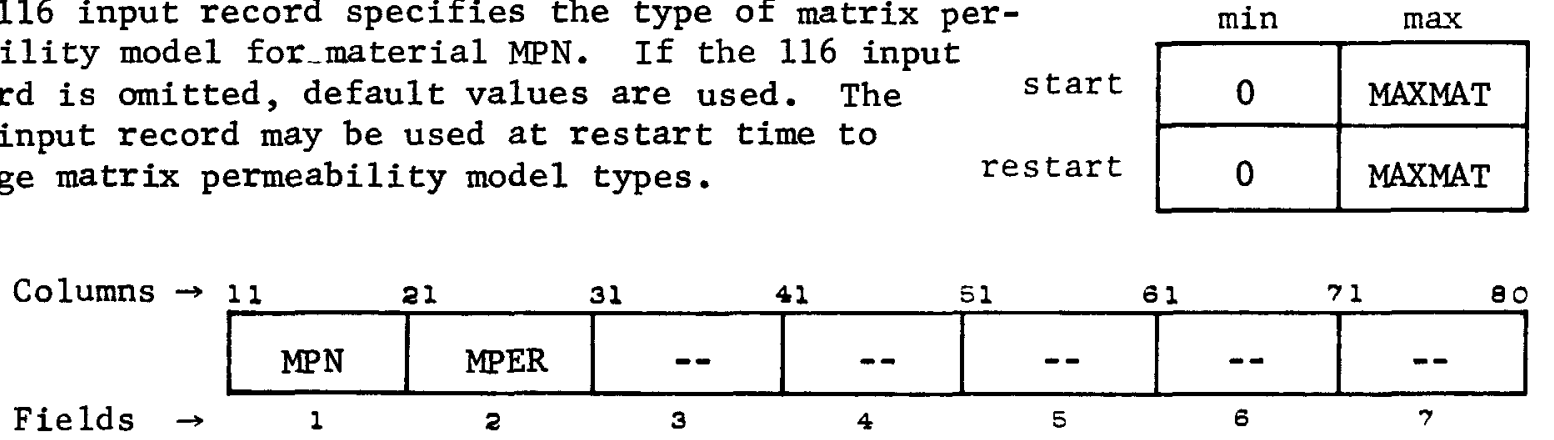

Input

Field Variable Number Name Description of Input Variable

$1 \quad$ MPN Material property number. See Field Number 1 on TYPE 111 record.

2 MPER

Permeability model type. The value of MPER identifies the type of matrix permeability model for material MPN. The matrix permeability model defines a relationship for matrix permeability as a function of thermodynamic variables and time. The value of MPER must be integral and must be greater than or equal to 1 and less than or equal to MAXCON. (See Appendix "Maximums".) If MPER is out of this range, a RGEERR message is written on the print and the message files and the program terminates immediately in subroutine MATINP. (See Appendix "Messages".) The following definitions apply:

$$
\begin{aligned}
& =1.0 \\
& =2.0 \\
& =3.0 \\
& =4.0 \\
& =5.0 \\
& =6.0- \\
& 10.0
\end{aligned}
$$

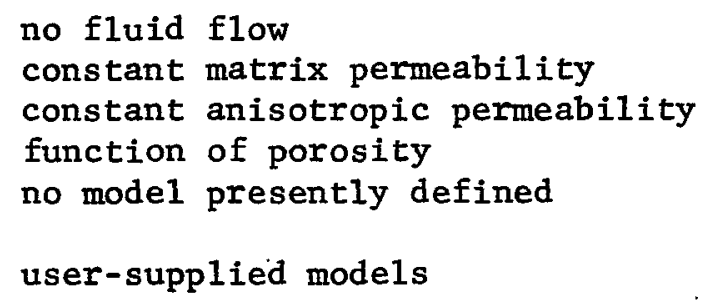

A description of each model is presented in Appendix "Material Models". Coefficients for a particular model are entered on input records 181 and 182 .

3-7 -- Fields 3-7 must be left blank or the FLDCHK message is written on the print and the message files and the program terminates at the end of the GENERATOR phase group. (See Appendix "Messages".) 
Page 1 of 1

The 117 input record specifies the type of porosity model for material MPN. If the 117 input record is omitted, default values are used. The 117 input record may be used at restart time to change porosity model types.

\begin{tabular}{c|c|c|}
\multicolumn{1}{c}{$\operatorname{start}$} & \multicolumn{1}{c}{$\min$} & \multicolumn{1}{c}{$\max$} \\
\cline { 2 - 3 } restart & 0 & MAXMAT \\
\cline { 2 - 3 } & 0 & MAXMAT \\
\cline { 2 - 3 } & &
\end{tabular}

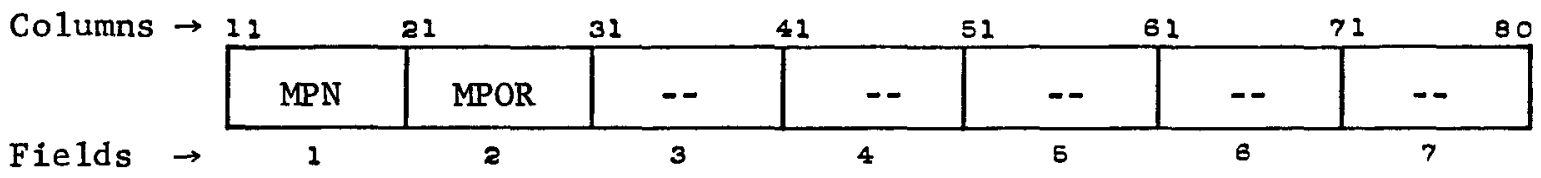

Input

Field Variable

Number Name

Description of Input Variable

$1 \quad$ MPN

Material property number. See Field Number 1 on TYPE 111 record.

$2 \quad$ MPOR

3-7 -- Fields 3-7 must be left blank or the FLDCHK message is written on the print and the message files and the program terminates at the end of the GENERATOR phase group. (See Appendix "Messages".)

A description of each model is presented in Appendix "Material Mode1s". Coefficients for a particular model are entered on input records 183 and 184 . porosity moder for relationship for porosity as a function of thermodynamic variables and time. The value of MPOR must be integral and must be greater than or equal to $I$ and less than or equal to MAXCON. (See Appendix "Maximums".) If MPOR is out of this range, a RGEER message is written on the print and the message files and the program terminates immediately in subroutine MATINP. (See Appendix "Messages".) The following definitions apply:

$$
\begin{aligned}
= & 1.0 \\
= & 2.0 \\
= & 3.0 \\
= & 4.0 \\
= & 5.0 \\
= & 6.0- \\
& 10.0
\end{aligned}
$$$$
\text { no porosity (default) }
$$$$
\text { constant porosity }
$$$$
\text { function of volumetric strain, fluid pressure, }
$$
and temperature no model presently defined no model presently defined

user-supplied models 


$$
\text { Page } 1 \text { of } 1
$$

The 121 input record specifies the reference densities for the equation of state for material MPN. The 121 record is absolutely required for each equation-of-state model. The 121 input record may be used at restart time to change the reference densities.
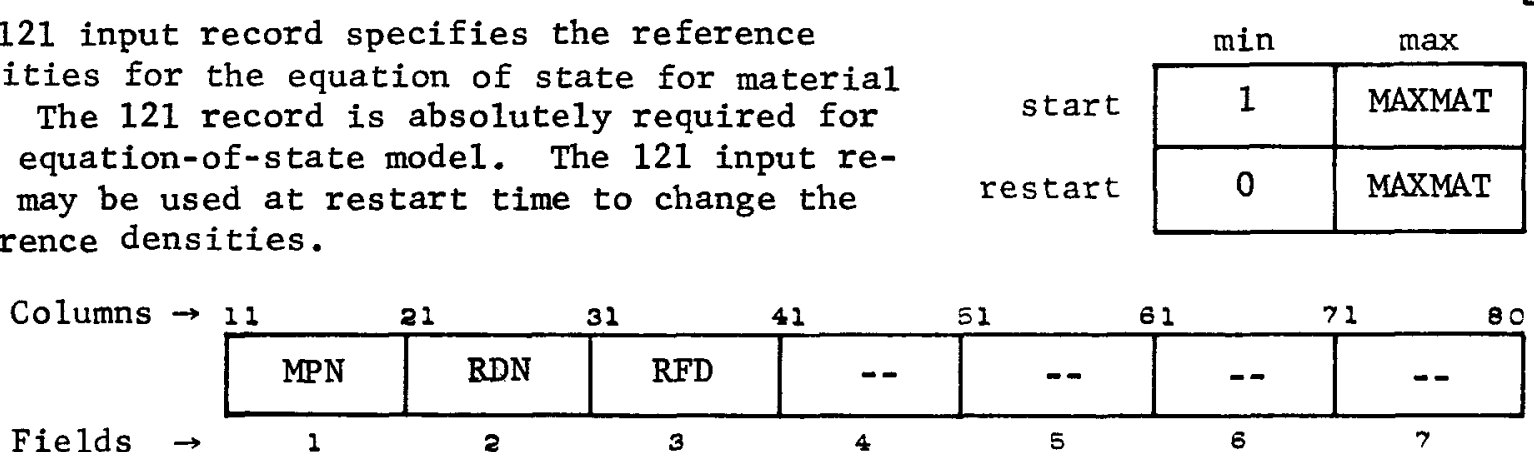

Input

Field Variable Number Name Description of Input Variable

1 MPN Material property number. See Field Number 1 on TYPE 111 record.

2 RDN Reference density. The value of RDN must be a positive number greater than SMLNUM. If RDN is less than SMLNUM a material is assumed not to exist. The value of RDN is often chosen to be that of the initial density. There is no default value.

$3 \quad$ RFD

Reference fluid density. The value of RFD must be a positive number greater than SMLNUM. If RFD is less than SMLNUM a material is assumed not to exist. The value of RFD is often chosen to be that of the initial density. There is no default value.

4-7 -- $\quad$ Fields 4-7 must be left blank or the FLDCHK message is written on the print and the message files and the program terminates at the end of the GENERATOR phase group. (See Appendix "Messages".)

B-8 Rev. $\quad \begin{gathered}\text { TYPE } \\ 121\end{gathered}$


The 124 input record specifies pore pressure parameters for the fluid pore pressure model specified on the 111 input record. If the 124 input record is omitted, default values are used. The 124 input record may be used at restart time to change equations-of-state parameters.

\begin{tabular}{c|c|c|}
\multicolumn{1}{c}{$\operatorname{c}$ min } & \multicolumn{1}{c}{ max } \\
\cline { 2 - 3 } restart & 0 & MAXMAT \\
\cline { 2 - 3 } & 0 & MAXMAT \\
\cline { 2 - 3 } & &
\end{tabular}

\begin{tabular}{|c|c|c|c|c|c|c|c|}
\hline \multirow[t]{2}{*}{ Columns $\rightarrow$} & \multicolumn{2}{|c|}{22} & 32 & 1 & 51 & 31 & 1 \\
\hline & MPN & FPPO & FPP I & FPP2 & FPP 3 & EPP4 & - \\
\hline
\end{tabular}

Input

Field Variable

Number Name

Description of Input Variable

$1 \quad$ MPN

Material property number. See Field Number 1 on TYPE 111 record.

$2 \quad$ FPPO

Fluid pore pressure parameter 0. (See Appendix "Material Models".) Default value is 0 .

3 FPP1 Fluid pore pressure parameter 1. (See Appendix "Material Models".) Default value is 0 .

4 FPP2 Fluid pore pressure parameter 2. (See Appendix "Material Models".) Default value is 0 .

5 FPP3 Fluid pore pressure parameter 3. (See Appendix "Material Mode1s".) Default value is 0 .

6 FPP4 Fluid pore pressure parameter 4. (See Appendix "Material Models".) Default value is 0 .

$7 \quad$-- $\quad$ Field 7 must be left blank or the FLDCHK message is written on the print and the message files and the program terminates at the end of the GENERATOR phase group. (See Appendix "Messages".) 


$$
\text { Page } 1 \text { of } 1
$$

The 181 input record specifies fluid permeability parameters for the fluid permeability model specified on the 116 input record. If the 181 input record is omitted, default values are used. The 181 input record may be used at restart time to change fluid permeability parameters.

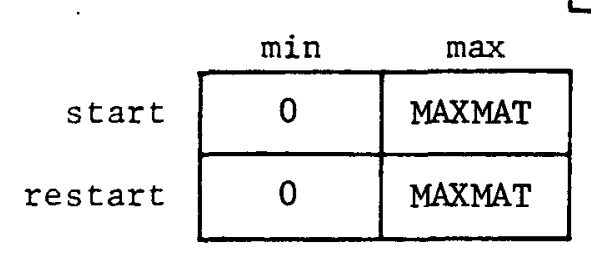

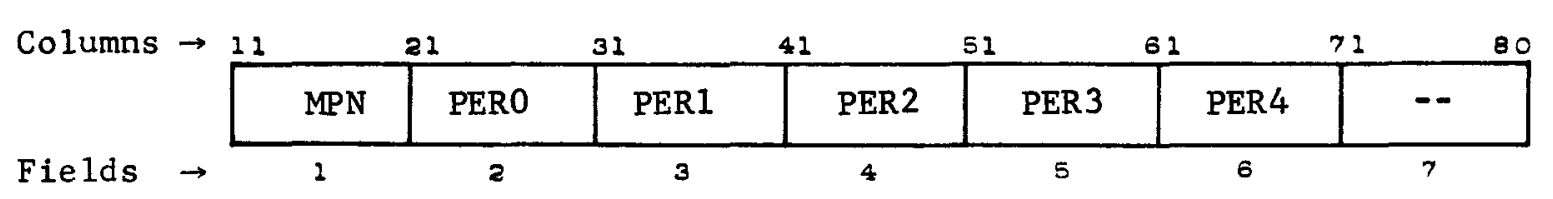

Input

Field Variable

Number Name

$1 \quad M P N$

2 PERO

$3 \quad$ PER 1

$4 \quad$ PER2

$5 \quad$ PER3

$6 \quad$ PER4

$7 \quad \ldots$
Description of Input Variable

Material property number. See Field Number 1 on TYPE 111 record.

Fluid permeability parameter 0. (See Appendix "Material Models".) Default value is 0 .

Fluid permeability parameter 1. (See Appendix "Material Models".) Default value is 0 .

Fluid permeability parameter 2. (See Appendix "Material Models".) Default value is 0 .

Fluid permeability parameter 3. (See Appendix "Material Models".) Default value is 0 .

Fluid permeability parameter 4. (See Appendix "Material Models".) Default value is 0 .

Field 7 must be left blank or the FLDCHK message is written on the print and the message files and the program terminates at the end of the GENERATOR phase group. (See Appendix "Messages".) 
The 182 input record specifies fluid permeability parameters for the fluid permeability model specifield on the 116 input record. If the 182 input record is omitted, default values are used. The 182 input record may be used at restart time to change fluid permeability parameters.

\begin{tabular}{c|c|c|}
\multirow{1}{*}{$\sin$ start } & \multicolumn{1}{c}{ min } & \multicolumn{1}{c}{$\max$} \\
\cline { 2 - 3 } restart & 0 & MAXMAT \\
\cline { 2 - 3 } & 0 & MAXMAT \\
\cline { 2 - 3 } & &
\end{tabular}

\begin{tabular}{|c|c|c|c|c|c|c|c|}
\hline \multirow[t]{2}{*}{ Columns - } & \multicolumn{2}{|c|}{21} & 32 & 1 & \multicolumn{2}{|c|}{$11 \quad 61$} & 72 \\
\hline & MPN & PER5 & PER6 & PER7 & PER 8 & PER 9 & -- \\
\hline
\end{tabular}

Input

Field Variable

Number Name

Description of Input Variable

1 MPN Material property number. See Field Number 1 on TYPE 111 record.

2 PER5 Fluid permeability parameter 5. (See Appendix "Material Models".) Default value is 0 .

3 PER6 Fluid permeability parameter 6. (See Appendix "Material Models".) Default value is 0 .

4 PER7 Fluid permeability parameter 7. (See Appendix "Material Models".) Default value is 0 .

5 PER8 Fluid permeability parameter 8. (See Appendix "Material Models".) Default value is 0 .

6 PER9 Fluid permeability parameter 9. (See Appendix "Material Models".) Default value is 0 .

7 -- Field 7 must be left blank or the FLDCHK message is written on the print and the message files and the program terminates at the end of the GENERATOR phase group. (See Appendix "Messages".) 
The 183 input record specifies porosity parameters for the porosity parameter model specified on the 117 input record. If the 183 input record is omitted, default values are used. The 183 input record may be used at restart time to change

\begin{tabular}{c|c|c|}
\multirow{1}{*}{ start } & \multicolumn{1}{c}{$\min$} & \multicolumn{1}{c}{$\max$} \\
\cline { 2 - 3 } restart & 0 & MAXMAT \\
\cline { 2 - 3 } & 0 & MAXMAT \\
\cline { 2 - 3 } & &
\end{tabular}
porosity parameters.

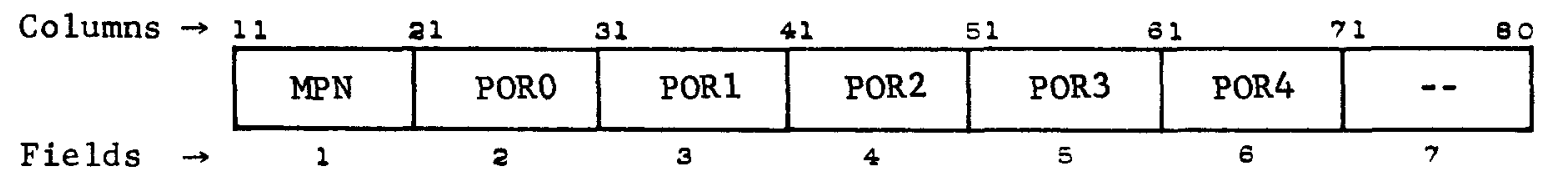

Input

Field Variable

Number Name Description of Input Variable

1 MPN

Material property number. See Field Number 1 on TYPE 111 record.

2 PORO

Porosity parameter 0. (See Appendix "Material Models".) Default value is 0 .

3 POR1 Porosity parameter 1. (See Appendix "Material Models".) Default value is 0 .

4 POR2 Porosity parameter 2. (See Appendix "Material Mode1s".) Default value is 0 .

5 POR3 Porosity parameter 3. (See Appendix "Material Models".) Default value is 0 .

6 POR4 Porosity parameter 4. (See Appendix "Material Mode1s".) Default value is 0 .

7 -- Field 7 must be left blank or the FLDCHK message is written on the print and the message files and the program terminates at the end of the GENERATOR phase group. (See Appendix "Messages".) 
The 184 input record specifies porosity parameters for the porosity parameter model specified on the 117 input record. If the 184 input record is omitted, default values are used. The 184 input record may be used at restart time to change

Page 1 of 1

\begin{tabular}{c|c|c|}
\multicolumn{1}{c}{$\sin \operatorname{start}$} & \multicolumn{1}{c}{$\min$} & \multicolumn{1}{c}{$\max$} \\
\cline { 2 - 3 } restart & 0 & MAXMAT \\
\cline { 2 - 3 } & 0 & MAXMAT \\
\cline { 2 - 3 } & &
\end{tabular}
porosity parameters.

\begin{tabular}{l} 
Columns $\rightarrow$\begin{tabular}{|c|c|c|c|c|c|c|}
\hline MPN & POR5 & POR6 & POR7 & POR8 & POR9 & -- \\
\hline
\end{tabular} Fields $\rightarrow$ \\
\hline 1
\end{tabular}

Input

Field Variable

Number Name

$1 \quad$ MPN

$2 \quad$ POR 5

$3 \quad$ POR6

$4 \quad$ POR

$5 \quad$ POR 8

$6 \quad$ POR9
Description of Input Variable

Material property number. See Field Number 1 on TYPE 111 record.

Porosity parameter 5. (See Appendix "Material Models".) Default value is 0 .

Porosity parameter 6. (See Appendix "Material Mode1s".) Default value is 0 .

Porosity parameter 7. (See Appendix "Material Models".) Default value is 0 .

Porosity parameter 8. (See Appendix "Material Mode1s"..) Default value is 0 .

Porosity parameter 9. (See Appendix "Material Mode1s".) Default value is 0 .

Field 7 must be left blank or the FLDCHK message is written on the print and the message files and the program terminates at the end of the GENERATOR phase group. (See Appendix "Messages".) 


$$
\text { Page } 1 \text { of } 1
$$

The 351 input record specifies the initial relative fluid mass, porosity and $x$ and $y$ permeabilities, for zones in block number $L$. At least one 351 input record is required for all start runs. It may not be used at restart time.

\begin{tabular}{c|c|c|}
\multirow{1}{*}{$\operatorname{start}$} & \multicolumn{1}{c}{$\min$} & \multicolumn{1}{c}{$\max$} \\
\cline { 2 - 3 } restart & 1 & MAXBLK \\
\cline { 2 - 3 } & 0 & 0 \\
\cline { 2 - 3 } & &
\end{tabular}

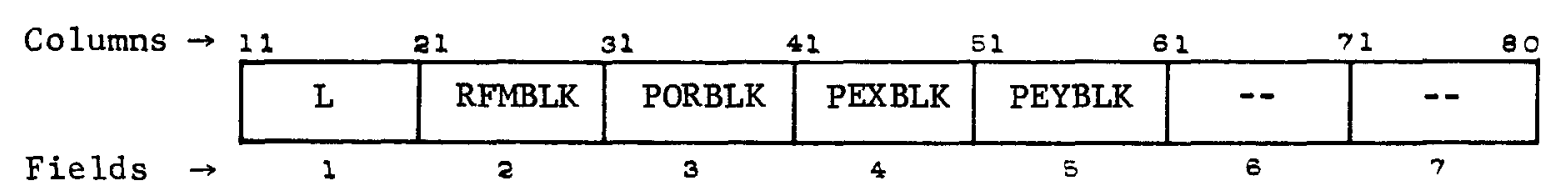

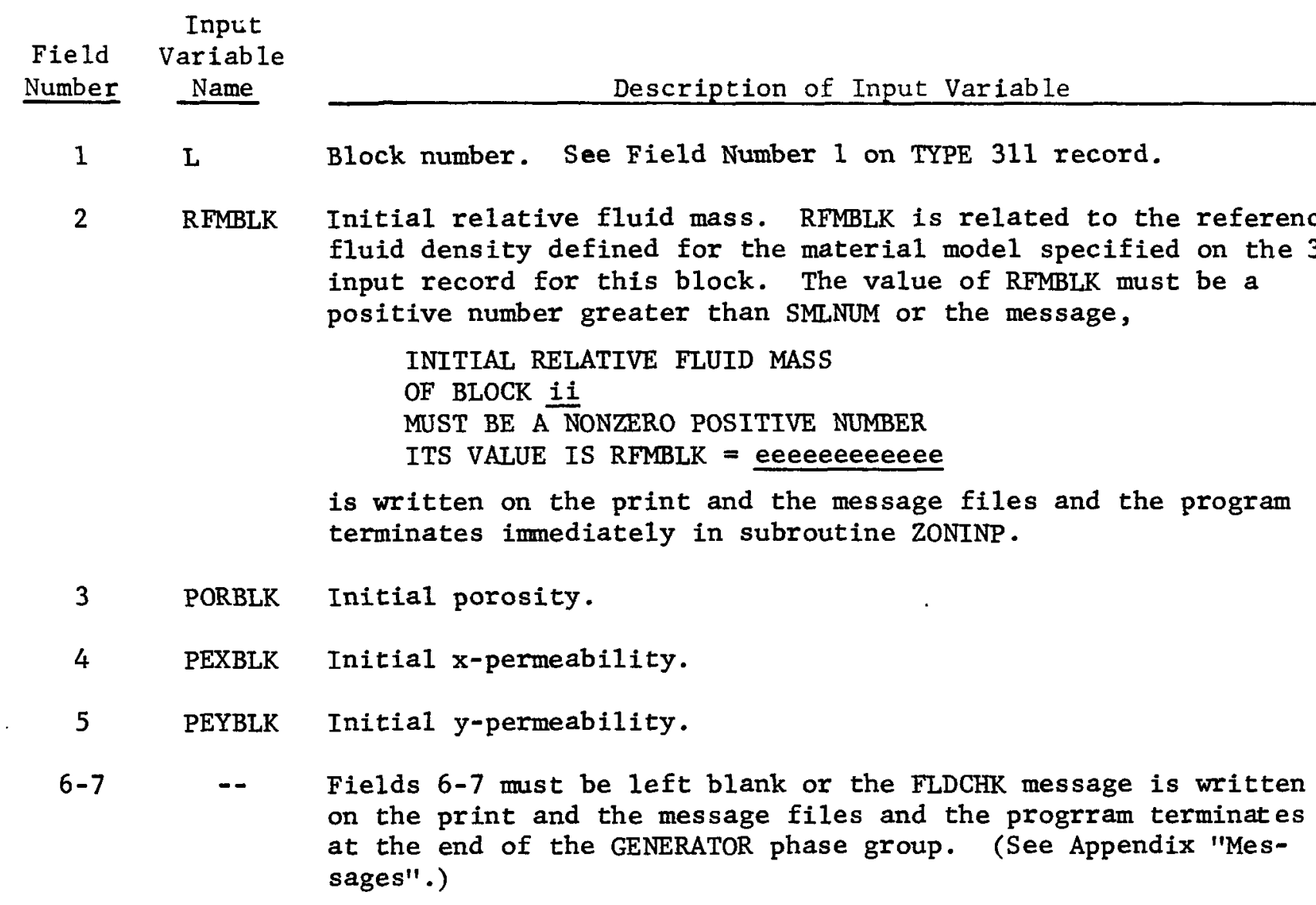

B-14 Rev. $\begin{gathered}\text { TYPE } \\ 351\end{gathered}$


Page 1 of 3

The 412 input record describes the properties of a contiguous sequence of boundary segments defined on the 411 input record.

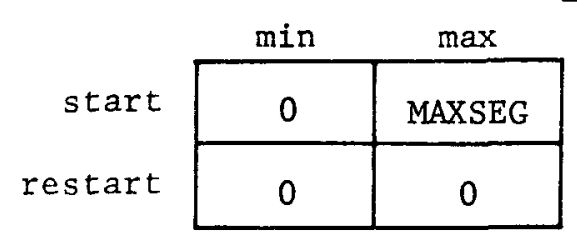

\begin{tabular}{|c|c|c|c|c|c|c|c|}
\hline \multirow{2}{*}{ Columns $\rightarrow$} & \multicolumn{2}{|c|}{31} & \multicolumn{2}{|l|}{32} & 1 & 1 & \\
\hline & LS & MPNBSG & NBMBSG & NBTBSG & NBHBBSG & NBVBSG & -- \\
\hline
\end{tabular}

$\begin{array}{cc}\text { Field } & \begin{array}{c}\text { Input } \\ \text { Variable } \\ \text { Number }\end{array} \quad \text { Name }\end{array}$

$1 \quad$ LS
Segment number. The value of LS uniquely identifies a sequence of boundary points defined by the grid point limits specified on record type 411 . The value of LS must be integral and greater than or equal to 1 and less than or equal to MAXSEG. (See Appendix "Maximums".) If LS is out of this range, a RGEERR message is written on the print and the message files and the program terminates immediately in subroutine BDYINP. (See Appendix "Messages".) If the segment is composed of only one point, then IBBGN = IBEND and JBBGN = JBEND. In two-dimensional problems, the boundary point limits must be defined counterclockwise in a right-handed $i, j$ space and must not cross a grid corner in $i, j$ space.

2 MPNBSG Material property number for this segment. The value of MPNBSG uniquely identifies a material model. A model consists of an equation of state and strength, explosive, and thermal descriptions; however, only the mechanical and thermal surface properties will be used. The value of MPNBSG must be integral and must be greater than or equal to 1 and less than or equal to MAXMAT. (See Appendix "Maximums".) If MPNBSG is out of this range, a RGEERR message is written on the print and the message files and the program terminates immediately in subroutine BDYINP. (See Appendix "Messages".) 
Page 2 of 3

\begin{tabular}{|c|c|c|c|c|c|c|c|}
\hline \multirow[t]{2}{*}{ Columns $\rightarrow$} & & 1 & 31 & 41 & 1 & & 80 \\
\hline & LS & MPNBSG & NBMBSG & NBTBSG & NBHBSG & NBVBSG & -- \\
\hline Fields $\rightarrow$ & 1 & 2 & 3 & 4 & 5 & 6 & 7 \\
\hline
\end{tabular}

Input

Field Variable Number Name

Description of Input Variable

$3 \quad$ NBMBSG

Momentum boundary number for this segment. The value of NBMBSG identifies the momentum boundary condition type. The value of NBMBSG must be integral and greater than or equal to 1 and less than or equal to 8 . If NBMBSG is out of this range, a RGEERR message is written on the print and the message files and the program terminates immediately in subroutine BDYINP. (See Appendix "Messages".) The following definitions apply:

\begin{tabular}{|c|c|}
\hline $\begin{array}{l}=1.0 \\
=2.0 \\
=3.0 \\
=4\end{array}$ & $\begin{array}{l}\text { interior } \\
\text { pressure history } \\
\text { free, } p=0.0 \\
\text { velocity history } \\
\text { fixed (rigid) } \\
\text { wall interaction } \\
\text { grid interaction }\end{array}$ \\
\hline
\end{tabular}

4 NBTBSG Thermal boundary number for this segment. The value of NBTBSG identifies the thermal boundary condition type. The value of NBTBSG must be integral and greater than or equal to 1 and less than or equal to 8 . If NBTBSG is out of this range, a RGEERR message is written on the print and the message files and the program terminates immediately in subroutine BDYINP. (See Appendix "Messages".) The following definitions apply:

\begin{tabular}{|c|c|}
\hline $\begin{array}{l}=1.0 \\
=2.0 \\
=3.0 \\
=4.0 \\
=5.0 \\
=6.0 \\
=7.0 \\
=8.0\end{array}$ & $\begin{array}{l}\text { interior } \\
\text { temperature history } \\
\text { isothermal, } \mathrm{T}=0.0 \\
\text { heat flux history } \\
\text { adiabatic } \\
\text { wall interaction } \\
\text { grid interaction } \\
\text { special }\end{array}$ \\
\hline
\end{tabular}

* When a pressure history boundary point in a particular cycle is outside of the range of the function defined on the 431 input record, it automatically becomes a free point for that cycle. When a velocity history point is outside of its range as defined on the 441 input record, it is permanently changed to a free point for all time.

$* *$ When a temperature history boundary point in a particular cycle is outside of the range of the function defined on the 451 input record, it automatically becomes a isothermal point where $T=0$ for that cycle. When a heat flux history point is outside of its range as defined on the 451 input record, it is permanently changed to $a$ isothermal point where $\mathrm{T}=0$ for all time.

Rev. 


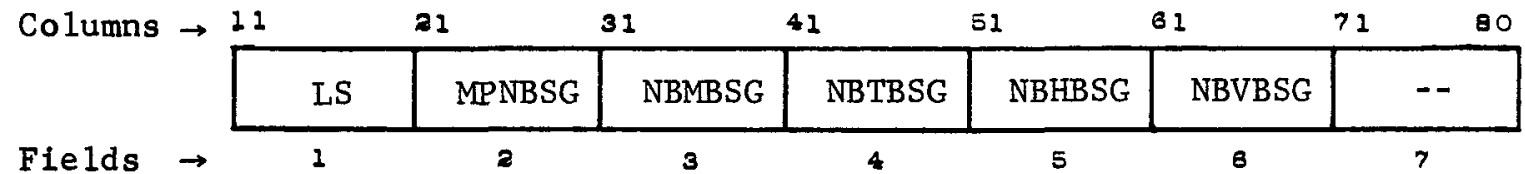

Input

Field Variable

Number Name

Description of Input Variable

$5 \quad$ NBHBSG

Hydraulic boundary number for this segment. The value of NBHBSG identifies the hydraulic boundary conditions type. The value of NBHBSG must be integral and greater than or equal to 1 and less than or equal to 8 . If NBHBSG is out of this range, a RGEERR message is written on the print and the message files and the program terminates immediately in subroutine BDYINP. (See Appendix "Messages".) The following definitions apply:

$$
\begin{aligned}
& =1.0 \\
& =2.0 \\
& =3.0 \\
& =4.0 \\
& =5.0 \\
& =6.0 \\
& =7.0 \\
& =8.0
\end{aligned}
$$

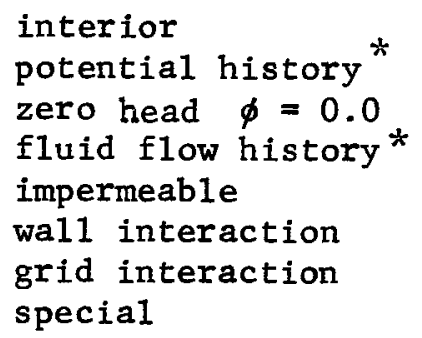

$6 \quad$ NBVBSG

Index of boundary array. The value of NBVBSG specifies the index location of boundary history model data for a grid point which has a history specification; or it specifies the greater wall point number of the wall point segment that it is touching or near, for a grid point which interacts with wall points; or it specifies the closest $i$ value of the interaction row in the other grid for a grid point which interacts with grid points in another grid. When the grid point is not a history, wall-interacting, or grid-interacting boundary point, leave NBVBSG blank. The value of NBVBSG must be integral and greater than or equal to 0 and less than or equal to MAXBDY. (See Appendix "Maximums".) If NBVBSG is out of this range, a RGEERR message is written on the print and the message files and the program terminates immediately in subroutines BDYINP. (See Appendix "Messages".)

7 -- Field 7 must be left blank or the FLDCHK message is written on the print and the message files and the program terminates at the end of the GENERATOR phase group. (See Appendix "Messages".)

*Wen a potential history boundary point in a particular cycle is outside of the range of the function defined on the 471 input record, it automatically becomes a zero head point where $\phi=0$ for that cycle. When a fluid flow history point is outside of its range as defined on the 473 input record, it is permanently changed to a zero head point where $\phi=0$ for all time. 
The 424 input record is required for each boundary point whose hydraulic boundary is a specified time history input. It is used in conjunction with the 421 input record in $1 D$ and the 412 input record in $2 \mathrm{D}$.

\begin{tabular}{c|c|c|}
\multirow{1}{*}{$\operatorname{start}$} & \multicolumn{1}{c}{$\min$} & \multicolumn{1}{c}{$\max$} \\
\cline { 2 - 3 } restart & 0 & MAXBDY \\
\cline { 2 - 3 } & 0 & MAXBDY \\
\cline { 2 - 3 } & &
\end{tabular}

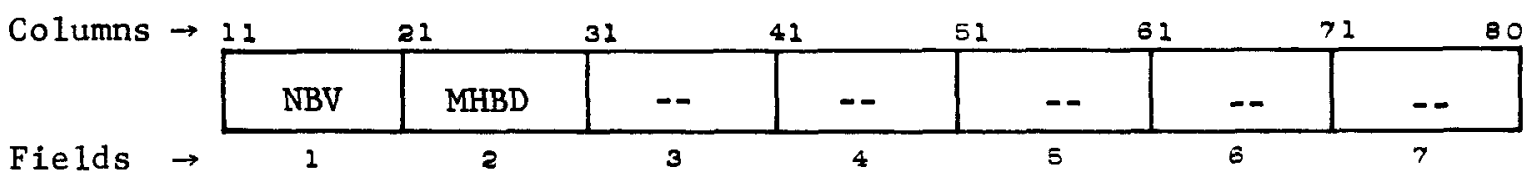

Input

Field Variable

Number Name

Description of Input Variable

$1 \quad \mathrm{NBV}$

Index of boundary array. The value of NBV specifies the index location of boundary history model data for a grid point in the boundary values array. When no history data is required, NBV is left blank. The value of NBV must be integral and greater than or equal to 0 and less than or equal to MAXBDY. (See Appendix "Maximums".) If NBV is out of this range, a RGEERR message is written on the print and the message files and the program terminates immediately in subroutine BDYINP. (See Appendix "Messages".)

2 MHBD Hydraulic boundary model number. The value of MHBD defines the array location of the function ocefficients, model, and range defined on input records 471 thru 476. The value of MHBD must be integral and greater than or equal to 1 and less than or equal to MAXTBD. (See Appendix "Maximums".) If MHBD is out of this range, a RGEERR message is written on the print and the message files and the program terminates immediately in subroutine BDYINP. (See Appendix "Messages".)

3-7 -- Fields 3-7 must be left blank or the FLDCHK message is written on the print and the message files and the program terminates at the end of the GENERATOR phase group. (See Appendix "Messages".) 
Page 1 of 2

A 471 input record is required to specify a function type and a range for a potential boundary.
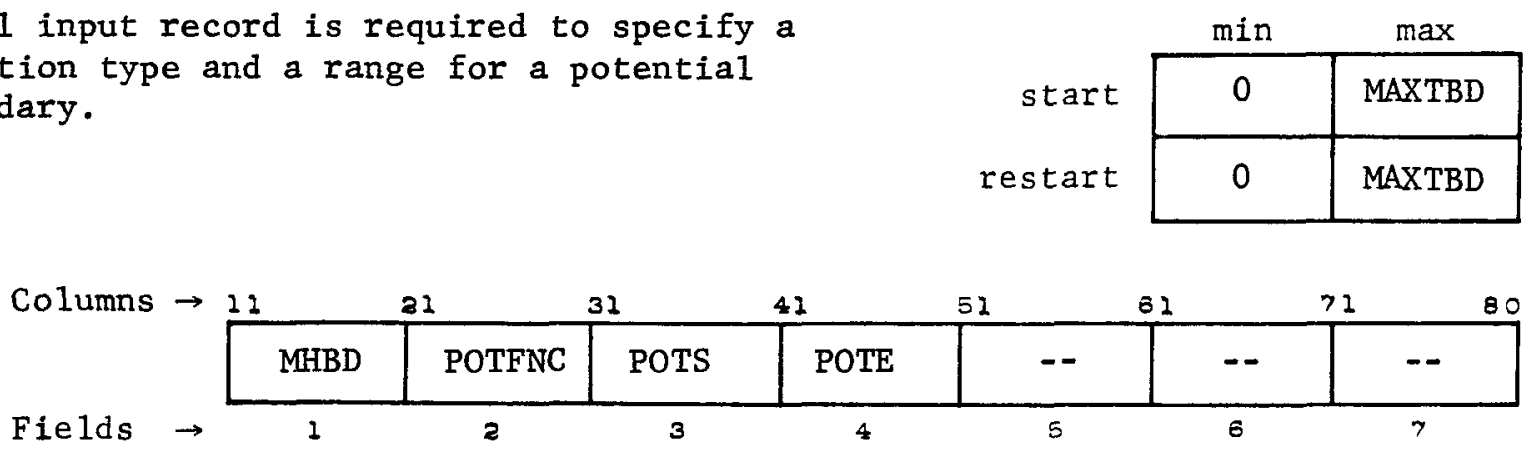

Input

Field Variable

Number Name

Description of Input Variable

1 MHBD

Hydraulic boundary model number. The value of MHBD defines the array location of the function coefficients, model, and range defined on input records 471 thru 476. The value of MHBD must be integral and greater than or equal to 1 and less than or equal to MAXTBD. (See Appendix "Maximums".) If MHBD is out of this range, a RGEERR message is written on the print and the message files and the program terminates immediately in subroutine BDYINP. (See Appendix "Messages".)

2 POTFNC Function type for potential history modeling. The value of POTFNC specifies the type of function of time. The value of POTFNC must be integral and greater than or equal to 1 and less than or equal to 10 . If POTFNC is out of this range, a RGEERR message is written on the print and the message files and the program terminates immediately in subroutine BDYINP. (See Appendix "Messages".) The following definitions apply:

$\begin{array}{ll}=1.0 & \text { polynomial } \\ =2.0 & \text { rational } \\ =3.0 & \text { exponential 非 } \\ =4.0 & \text { exponential 非 } \\ =5.0 & \text { exponential 非 } \\ =6.0 & \text { trigonometric } \# 1 \\ =7.0 & \text { trigonometric } \#^{2} 2 \\ =8.0 & \text { (none) } \\ =9.0 & \text { user-supplied } \\ =10.0 & \text { user-supplied }\end{array}$

See Appendix "Functions" for a description of the available function types.

3 POTS Start of time range for potential function. The value of POTS is a positive number. The value of problem time must be greater than or equal to POTS and less than POTE for the function to be activated. 


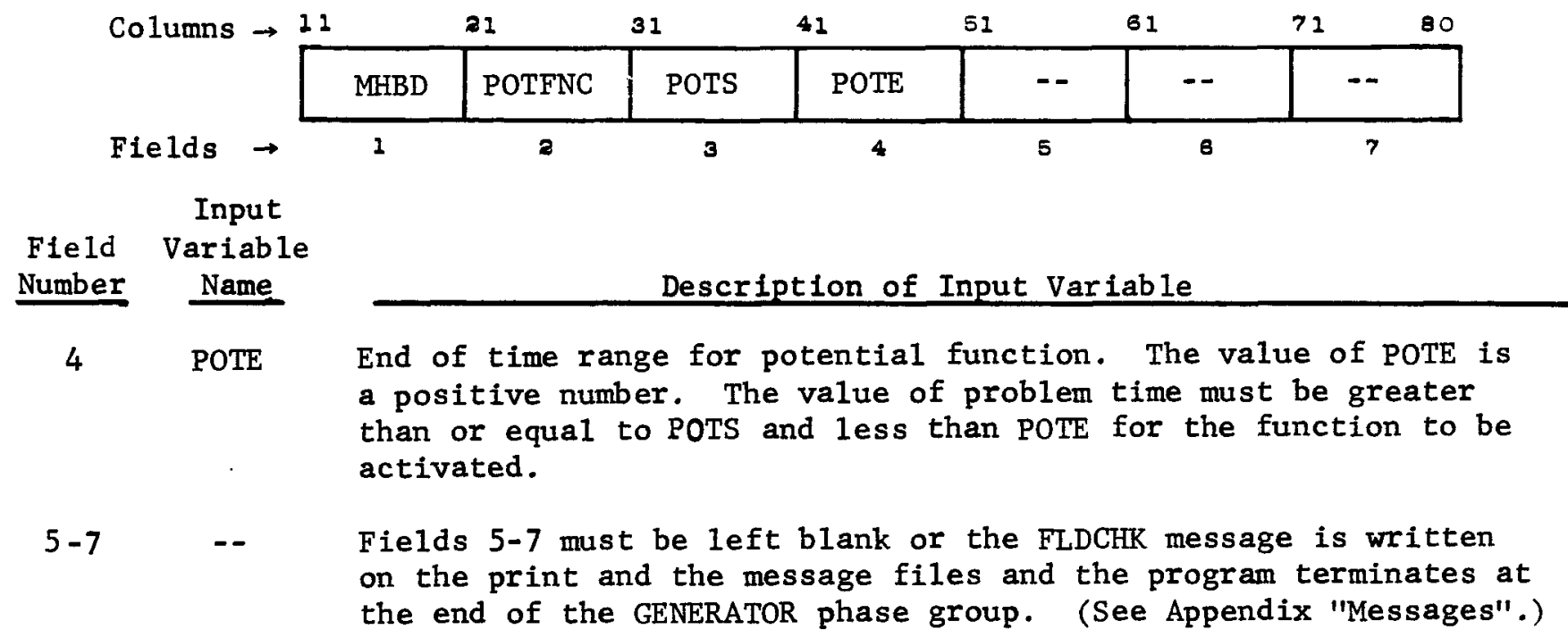


Page 1 of 1

The 472 input record is required to specify function coefficients for a potential boundary.

\begin{tabular}{c|c|c|}
\multirow{1}{*}{$\operatorname{start}$} & \multicolumn{1}{c}{ min } & \multicolumn{1}{c}{$\max$} \\
\cline { 2 - 3 } restart & 0 & MAXTBD \\
\cline { 2 - 3 } & 0 & MAXTBD \\
\cline { 2 - 3 } & &
\end{tabular}

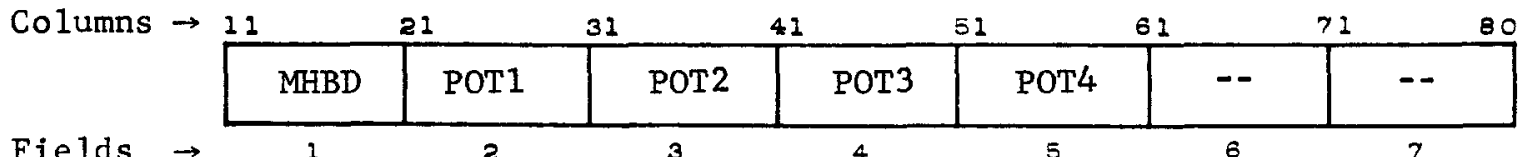

Input

Field Variable Number Name Description of Input Variable

$1 \quad$ MHBD

-Hydraulic boundary model number. The value of MHBD defines the array location of the function coefficients, model, and range defined on input records 471 thru 476. The value of MHBD must be integral and greater than or equal to 1 and less than or equal to MAXTBD. (See Appendix "Maximums".) If MHBD is out of this range, a RGEERR message is written on the print and the message files and the program terminates immediately in subroutine BDYINP. (See Appendix "Messages".)

2 POT1 Potential coefficient number 1 . Default value is 0.

3 РОт2 Potential coefficient number 2. Default value is 0.

4 РОт3 Potential coefficient number 3. Default value is 0.

5 POT4 Potential coefficient number 4. Default value is 0.

6-7 -- Fields 6-7 must be left blank or the FLDCHK message is written on the print and the message files and the program terminates at the end of the GENERATOR phase group. (See Appendix "Messages".) 


\section{Page 1 of 2}

The 473 input record is required to specify a function type and a range for an $x$-fluid flow boundary.

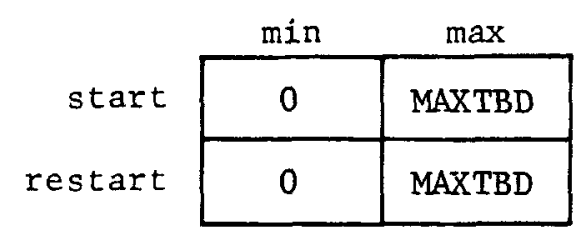

\begin{tabular}{|c|c|c|c|c|c|c|c|}
\hline Columns - & & 1 & 1 & 1 & 51 & & \\
\hline & MHBD & XFFFNC & XFFS & XFFE & -- & -- & -- \\
\hline
\end{tabular}

Input

Field Variable

Number Name Description of Input Variab1e

1 MHBD Hydraulic boundary model number. The value of MHBD defines the array location of the function coefficients, model, and range defined on input records 471 thru 476 . The value of MHBD must be integral and greater than or equal to 1 and less than or equal to MAXTBD. (See Appendix "Maximums".) If MHBD is out of this range, a RGEERR message is written on the print and the message files and the program terminates immediately in subroutine BDYINP. (See Appendix "Messages".)

2 XFFFNC Function type for $x$-fluid flow history modeling. The value of XFFFNC specifies the type of function of time. The value of XFFFNC must be integral and greater than or equal to 1 and less than or equal to 10 . If XFFFNC is out this range, a RGEERR message is written on the print and the message files and the program terminates immediately in subroutine BDYINP. (See Appendix "Messages".) The following definitions apply:

\begin{tabular}{|c|c|}
\hline $\begin{array}{l}=1.0 \\
=2.0 \\
=3.0 \\
=4.0 \\
=5.0 \\
=6.0 \\
=7.0 \\
=8.0 \\
=10.0\end{array}$ & $\begin{array}{l}\text { polynomial } \\
\text { rational } \\
\text { exponential \#1 } \\
\text { exponential 非 } \\
\text { exponential } \# 3 \\
\text { trigonometric } \# 1 \\
\text { trigonometric } 12 \\
\text { (none) } \\
\text { user-supplied } \\
\text { user-supplied }\end{array}$ \\
\hline
\end{tabular}

See Appendix "Functions" for a description of the available function types.

3 XFFS Start of time range of $x$-fluid flow function. The value of XFFS is a positive number. The value of problem time must be greater than or equal to XFFS and less than XFFE for the function to be activated. 


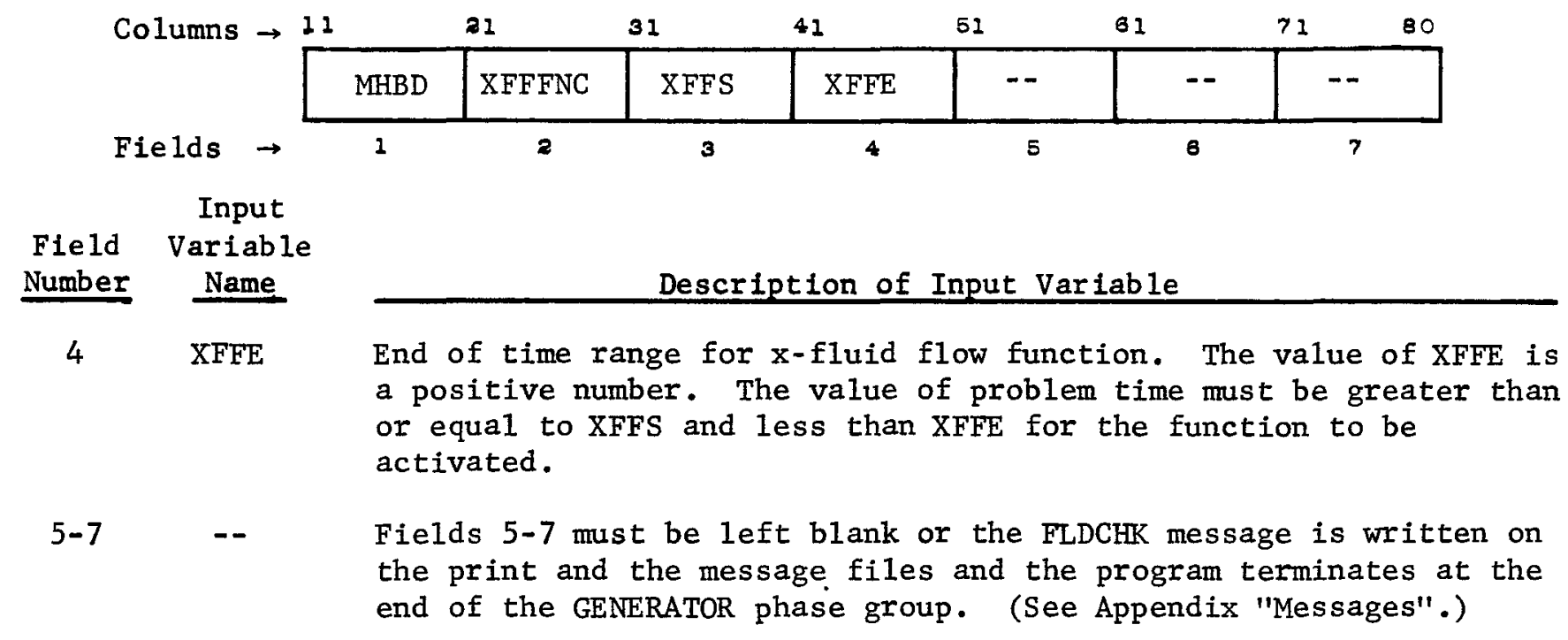

Rev. 


$$
\text { Page } 1 \text { of } 1
$$

The 474 input record is required to specify function coefficients for an $x$-fluid flow boundary.
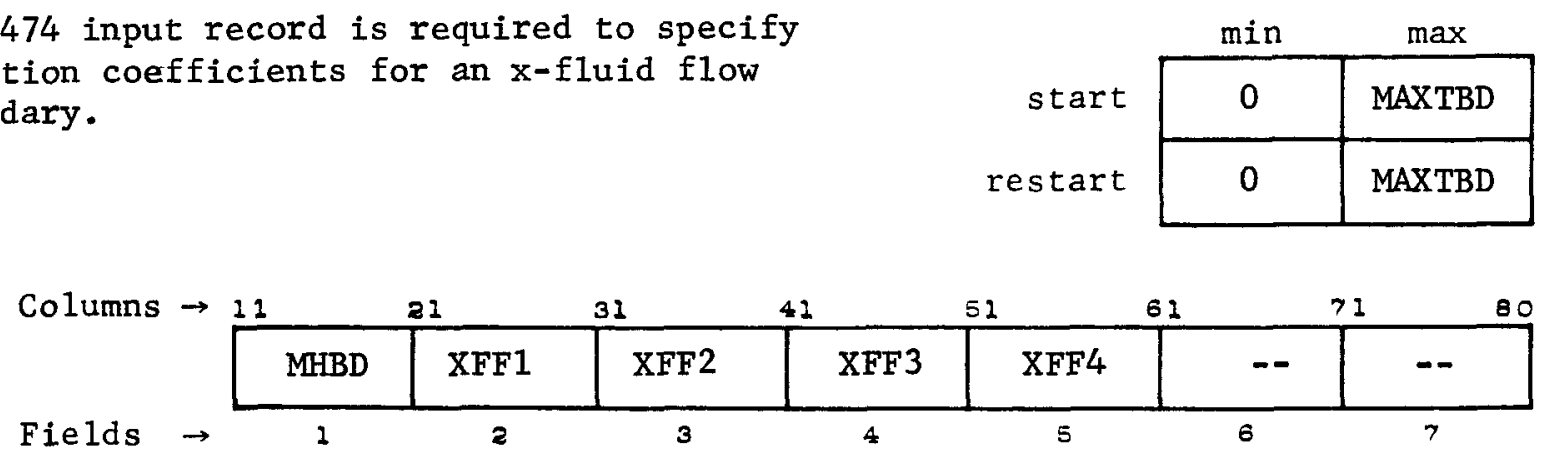

Input

Field Variable

Number Name

$1 \quad$ MHBD

Description of Input Variable

Hydraulic boundary model number. The value of MHBD defines the array location of the function coefficients, model, and range defined on input records 471 thru 476 . The value of MHBD must be integral and greater than or equal to 1 and less than or equal to MAXTBD. (See Appendix "Maximums".) If MHBD is out of this range, a RGEERR message is written on the print and the message files and the program terminates immediately in subroutine BDYINP. (See Appendix "Messages".)

$\begin{array}{llll}2 & \text { XFF1 } & \text { x-fluid flow coefficient number } 1 . \text { Default value is } 0 . \\ 3 & \text { XFF2 } & \text { x-fluid flow coefficient number } 2 . \text { Default value is } 0 . \\ 4 & \text { XFF3 } & \text { x-fluid flow coefficient number } 3 . \text { Default value is } 0 . \\ 5 & \text { XFF4 } & \text { x-fluid flow coefficient number } 4 . \text { Default value is } 0 .\end{array}$

6-7 -- Fields 6-7 must be left blank or the FLDCHK message is written on the print and the message files and the program terminates at the end of the GENERATOR phase group. (See Appendix "Messages".) 
Page 1 of 2

The 475 input record is required to specify a function type and a range for a $y$-fluid flow boundary.
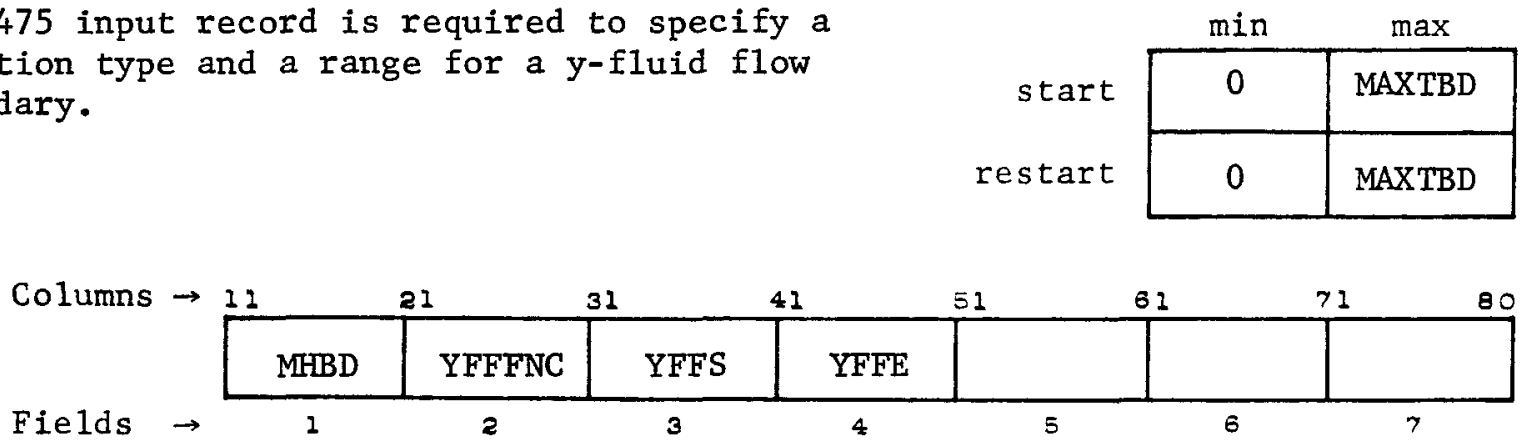

\begin{tabular}{|c|c|}
\hline $\begin{array}{l}\text { Field } \\
\text { Number }\end{array}$ & $\begin{array}{c}\text { Input } \\
\text { Variable } \\
\text { Name }\end{array}$ \\
\hline & MHBD \\
\hline
\end{tabular}

Hydraulic boundary model number. The value of MHBD defines the array location of the function coefficients, model, and range defined on input records 471 through 476 . The value of MHBD must be integral and greater than or equal to 1 and less than or equal to MAXTBD. (See Appendix "Maximums".) If MHBD is out of this range, a RGEERR message is written on the print and the message files and the program terminates immediately in subroutine BDYINP. (See Appendix "Messages".)

2 YFFFNC Function type for y-fluid flow history modeling. The value of YFFFNC specifies the type of function of time. The value of YFFFNC must be integral and greater than or equal to 1 and less than or equal to 10. If YFFFNC is out of this range, a RGEERR message is written on the print and the message files and the program terminates immediately in subroutine BDYINP. (See Appendix "Messages".) The following definitions apply:

$$
\begin{array}{lll}
=1.0 & & \text { polynomial } \\
=2.0 & & \text { rational } \\
=3.0 & & \text { exponential 非1 } \\
=4.0 & & \text { exponential 非 } \\
=5.0 & & \text { exponential 非 } \\
=6.0 & & \text { trigonometric } \# 1 \\
=7.0 & & \text { trigonometric } k_{2} \\
=8.0 & & \text { (none) } \\
=9.0 & & \text { user-supp1ied } \\
=10.0 & & \text { user-supp1ied }
\end{array}
$$

See Appendix "Functions" for a description of the available function types.

3 YFFS Start of time range of $y$-fluid flow function. The value of YFFS is a positive number. The value of problem time must be greater than or equal to YFFS and less than. YFFE for the function to be activated. 


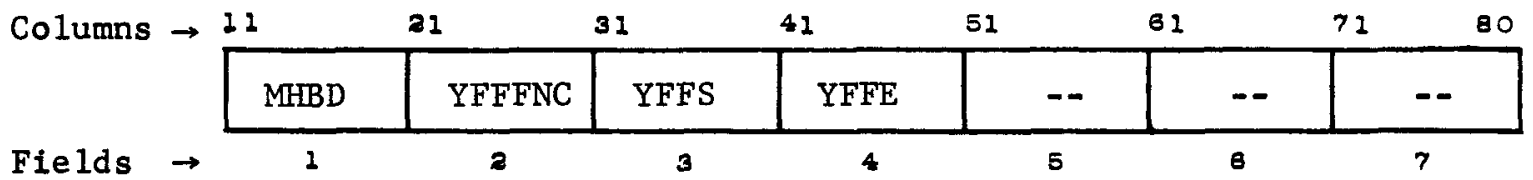

Input

Field Variable

Number Name

Description of Input Variable

4 YFFE End of time range for $y$-fluid flow function. The value of YFFE is a positive number. The value of problem time must be greater than or equal to YFFS and less than YFFE for the function to be activated.

5-7 -- Fields 5-7 must be left blank or the FLDCHK message is written on the print and the message files and the program terminates at the end of the GENERATOR phase group. (See Appendix "Messages".) 
The 476 input record is required to specify function coefficients for a $y$-fluid flow boundary.

\begin{tabular}{c|c|c|}
\multirow{1}{*}{$\operatorname{start}$} & \multicolumn{1}{c}{ min } & \multicolumn{1}{c}{$\max$} \\
\cline { 2 - 3 } & 0 & MAXTBD \\
\cline { 2 - 3 } restart & 0 & MAXTBD \\
\cline { 2 - 3 } & &
\end{tabular}

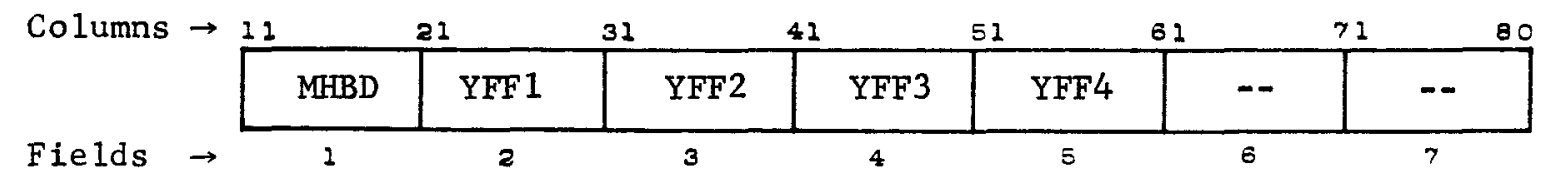

Input

Field Variable

Number Name

Description of Input Variable

1 MHBD Hydraulic boundary model number. The value of MHBD defines the array location of the function coefficients, model, and range defined on input records 471 thru 476. The value of MHBD must be integral and greater than or equal to 1 and less than or equal to MAXTBD. (See Appendix "Maximums".) The MHBD is out of this range, a RGEERR message is written on the print and the message files and the program terminates immediately in subroutine BDYINP. (See Appendix "Messages".)

2 YFF1 $y$-fluid flow coefficient number 1. Default value is 0.

3 YFF2 $y$-fluid flow coefficient number 2. Default value is 0.

4 YFF3 $y$-fluid flow coefficient number 3. Default value is 0.

5 YFF4 $y$-fluid flow coefficient number 4. Default value is 0 .

6-7 -- Fields 6-7 must be left blank or the LFDCHK message is written on the print and the message files and the program terminates at the end of the GENERATOR phase group. (See Appendix "Messages".) 
The 482 input record describes the properties of a contiguous sequence of wall point segments defined on the 481 input record. The 482 input record may not be used at restart time.

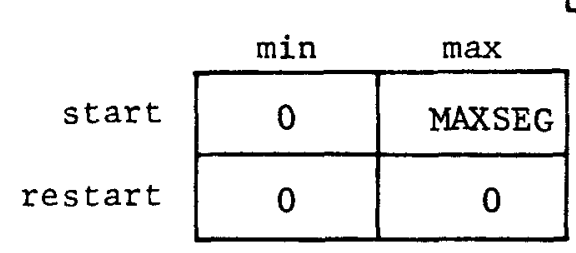

\begin{tabular}{|c|c|c|c|c|c|c|c|}
\hline \multirow{2}{*}{ Columns $\rightarrow$} & & 31 & 32 & \pm 1 & 51 & & \\
\hline & LS & MPNWSG & NBMWSG & NBTWSG & NBHWSG & NBVWSG & DWLWSG \\
\hline
\end{tabular}

Input

Field Variable

Number Name

Description of Input Variable

1 LS

2 MPNWS Material property number for this sequence. The value of MPNWSG uniquely identifies a material model. A model consists of an equation of state and strength, explosive, and thermal descriptions; however, only the mechanical and thermal surface properties will be used. The value of MPNWSG must be integral and must be greater than or equal to 1 and less than or equal to MAXMAT. (See Appendix "Maximums".) If MPNWSG is out of this range, a RGEERR message is writen on the print and the message files and the program terminates in subroutine BDYINP. (See Appendix "Messages".)

3 NBMWSG Momentum boundary number for this sequence. The value of NBMWSG identifies the momentum boundary condition type. The value of NBMWSG must be integral and greater than or equal to 4 and less than or equal to 5 or equal to 8 . If NBMWSG is out of this range, a RGEERR message is written on the print and the message files and the program terminates immediately in subroutine BDYINP. (See Appendix "Messages".) The following definitions apply:

$$
\begin{aligned}
& =4.0 \text { velocity history* } \\
& =5.0 \text { fixed (rigid) } \\
& =6.0 \text { special }
\end{aligned}
$$

*Wen a velocity history wäll point is outside of its range as defined on the 441 input record, it is permanently changed to a fixed wall point for all time

Rev. 


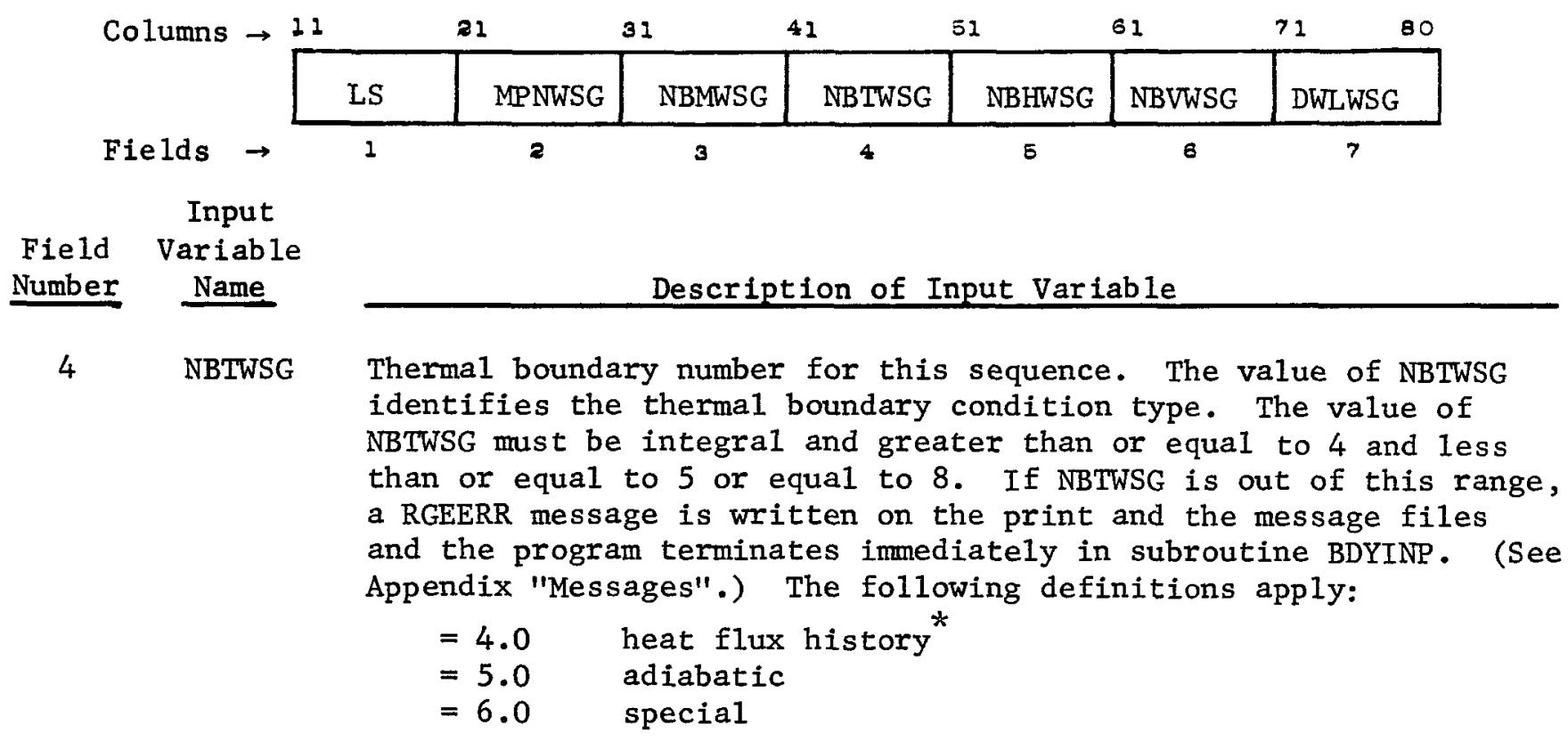

5 NBHWSG Hydraulic boundary number for this sequence. The value of NBHWSG identifies the hydraulic boundary condition type. The value of NBHWSG mist be integral and greater than or equal to 4 and less than or equal to 5 or equal to 8 . If NBHWSG is out of this range, a RGEERR message is written on the print and the message files and the program terminates immediately in subroutine BDYINP. (See Appendix "Messages".) The following definitions apply:

\begin{tabular}{|c|c|}
\hline $\begin{array}{l}=4.0 \\
=5.0 \\
=8.0\end{array}$ & $\begin{array}{l}\text { fluid flow history } \\
\text { impermeable } \\
\text { special }\end{array}$ \\
\hline
\end{tabular}

6 NBVWSG Index of boundary array. The value of NBVWSG specifies the index location of boundary history model data for a wall point in the boundary values array. When no history data are required, leave NBVWSG blank. The value of NBVWSG must be integral and greater than or equal to 0 and less than or equal to MAXBDY. (See Appendix "Maximums".) If NBVWSG is out of this range, a RGEERR message is written on the print and the message files and the program terminates immediately in subroutine BDYINP. (See Appendix "Messages".)

\footnotetext{
* When a heat flux history wall point is outside of its range as defined on the 461 input record, it is permanently changed to an adiabatic wall point for all time.

When a fluid flow history wall point is outside of its range as defined on the 473 input record, it is permanently changed to an impermeable wall point for all time.
} 


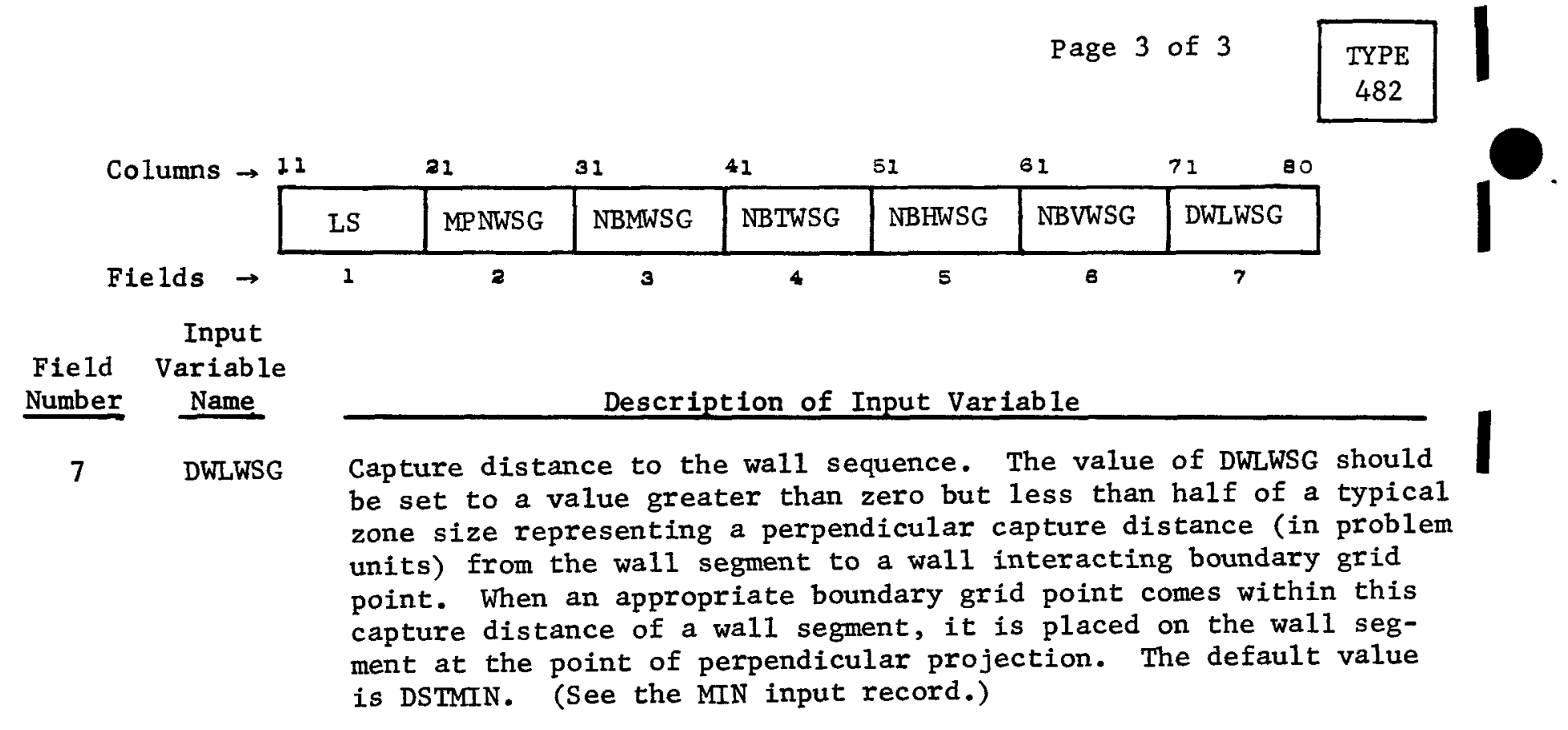

Rev. 
IDENTIFIERS (IDS)

$\begin{array}{cccccccccc}1 & 2 & 3 & 4 & 5 & 6 & 7 & 8 & 9 & 10 \\ \text { TIM } & \text { TIE } & \text { TDE } & \text { THE } & \text { TKE } & \text { TEG } & \text { TXM } & \text { TYM } & \text { TXK } & \text { TYK }\end{array}$

$\begin{array}{cccccccccc}11 & 12 & 13 & 14 & 15 & 16 & 17 & 18 & 19 & 20 \\ \mathrm{XPN} & \mathrm{XVL} & \mathrm{XAC} & \mathrm{YPN} & \mathrm{YVL} & \mathrm{YAC} & \mathrm{ZPN} & \text { ZVL } & \text { ZAC } & \text { DST }\end{array}$

$\begin{array}{llllllllll}21 & 22 & 23 & 24 & 25 & 26 & 27 & 28 & 29 & 30\end{array}$

GXK GYK GZK XHF YHF ZHF

$\begin{array}{cccccccccc}31 & 32 & 33 & 34 & 35 & 36 & 37 & 38 & 39 & 40 \\ \text { NOR } & \text { NBM } & \text { NBT } & \text { NBV } & \text { ACT } & \text { MPN } & \text { IND } & \text { NBH } & & \end{array}$

$\begin{array}{llllllllll}41 & 42 & 43 & 44 & 45 & 46 & 47 & 48 & 49 & 50\end{array}$

DLL RLV COM QDA $\quad$ VSR DNS ZMS TRV

$\begin{array}{cccccccccc}61 & 62 & 63 & 64 & 65 & 66 & 67 & 68 & 69 & 70 \\ \text { TMP } & \text { CON } & \text { SHC } & \text { PRH } & \text { AVS } & \text { SSP } & \text { BFS } & \text { IGN } & \text { XFF } & \text { YFF }\end{array}$

$\begin{array}{llllllllll}71 & 72 & 73 & 74 & 75 & 76 & 77 & 78 & 79 & 80\end{array}$ $\begin{array}{lllllllll}\text { TXX TYY } & \text { TZZ } & \text { TXY } & \text { TYZ } & \text { TZX } & \text { POR } & \text { FDN } & \text { FPP } & \text { POT }\end{array}$

$\begin{array}{cccccccccc}81 & 82 & 83 & 84 & 85 & 86 & 87 & 88 & 89 & 90 \\ \text { SXX } & \text { SYY } & \text { SZZ } & \text { SXY } & \text { SYZ } & \text { SZX } & \text { YLD } & \text { SHR } & \text { PEX } & \text { PEY }\end{array}$

$\begin{array}{ccccccccccc}91 & 92 & 93 & 94 & 95 & 96 & 97 & 98 & 99 & 100 & 101 \\ \text { EX1 } & \text { EX2 } & \text { EX3 } & \text { EX4 } & \text { EX5 } & \text { EX6 } & \text { XWL } & \text { YWL } & \text { NWO } & & \text { NCCYC }\end{array}$

Variables 1-10 are global variables, while the rest are either grid-point or zone-related. Variable definitions may be found in Appendix CVN of Volume 3 . 
•. 
MATERIAL MODELS (MAT)

There are several independent categories of information which must be specified to form the complete mechanical-thermal-hydraulic response model for a material. They are:

1. Thermodynamic mode1, $\mathrm{p}=\mathrm{p}(\mathrm{u}, \mathrm{V})$

2. Strength model, $\underline{=}=s(u, V)$

3. Energy release model, $\dot{u}=u(t)$

4. Heat flow model, $\dot{h}=\mathrm{h}(\mathrm{u}, \mathrm{V})$.

5. Artificial viscosity, $q=q\left(\frac{\dot{V}}{V}\right)$

6. Fluid thermodynamic model, $\mathrm{p}_{f}=\mathrm{p}_{f}(\mathrm{M}, \mathrm{T})$

7. Fluid flow model, $\dot{q}_{f}=q_{f}(M, \Phi)$

The minimum set of models must be chosen to satisfy boundary and initial conditions. The functions $p, s, u, h, p_{f}$ and $q_{f}$ may take on any convenient form -- analytic or tabular.

on the following pages, standard (i.e., already programmed) models for each modeling category are described according to the model-type value specified. Standard models use the function capability wherever possible.

Special (i.e., user-supplied) models are programmed into subroutines specifically set aside for special programming. The names of these usersupplied subroutines are always five characters as opposed to normal subroutine names which are composed of six characters. The names of special subroutines are formed by joining the prefix MY to a root of an appropriate model identifier, while the equivalent standard subroutine uses the root $\mathrm{ZON}$ as a prefix. The following user-supplied subroutines are available for material modeling: 


\begin{tabular}{lll}
$\begin{array}{c}\text { Standard } \\
\text { Subroutine }\end{array}$ & $\begin{array}{c}\text { User-supplied } \\
\text { Subroutine }\end{array}$ & Description \\
\hline ZONSDV & MYSDV & stress deviators \\
ZONYLD & MYYLD & yield stress \\
ZONSHR & MYSHR & shear modulus \\
ZONSPL & MYSPL & spall strength \\
ZONTMP & MYTMP & temperature \\
ZONCON & MYCON & conductivity \\
ZONSHC & MYSHC & specific heat capacity \\
ZONPRH & MYPRH & pressure equation of state \\
ZONERL & MYERL & energy release \\
ZONSSS & MYSSS & sound speed squared \\
ZONFPP & ZONFPP & fluid pressure equation of state \\
ZONPOT & ZONPOT & fluid hydraulic potential \\
ZONPER & ZONPER & permeability \\
ZONPOR & ZONPOR & porosity
\end{tabular}

Any combination of standard and/or user-supplied subroutines may be used to achieve a particular set of material-response characteristics. 
The calling sequence of the material modeling subroutines may be changed by re-programing subroutine ZONMDL (zone model). However, care must be taken that certain state variables in each zone be updated. These variables are summarized below.

\begin{tabular}{|c|c|c|c|c|}
\hline Variable Description & Symbol & $\begin{array}{c}\text { FORTRAN } \\
\text { Array } \\
\text { Name } \\
\end{array}$ & $\begin{array}{c}\text { Standard } \\
\text { Model } \\
\text { Subroutine } \\
\text { Name } \\
\end{array}$ & $\begin{array}{c}\text { User-Supplied } \\
\text { Mode1 } \\
\text { Subroutine } \\
\text { Name } \\
\end{array}$ \\
\hline Internal energy density & u & ZIE & ZONMDL & ZONMDL \\
\hline Pressure & $\mathrm{p}$ & PRH & ZONPRH & MYPRH \\
\hline Normal $\mathrm{xx}$ stress & $\sigma_{\mathrm{xx}}$ & $\operatorname{Txx}$ & ZONSTR & ZONSTR \\
\hline Normal yy stress & $\sigma_{\mathrm{yy}}$ & TYY & ZONSTR & ZONSTR \\
\hline Normal zz stress & $\sigma_{z z}$ & $\mathrm{TZZ}$ & ZONSTR & ZONSTR \\
\hline Shear xy stress & $\sigma_{x y}$ & $\operatorname{TXY}$ & ZONSTR & ZONSTR \\
\hline Specific heat capacity & $\mathrm{C}$ & SHC & ZONSHC & MYSHC \\
\hline Temperature & $T$ & TMP & ZONTMP & MYTMP \\
\hline Conductivity & $\mathrm{K}_{\mathrm{T}}$ & CON & ZONCON & MYCON \\
\hline Sound speed squared & $c^{2}$ & SSS & zONSSS & MYSSS \\
\hline Fluid density & $\rho_{\mathrm{f}}$ & ZFM & ZONZFM & ZONZFM \\
\hline Fluid pressure & $p_{f}$ & FPP & ZONEPP & ZONFPP \\
\hline Hydraulic potential & $\Phi$ & POT & ZONPOT & ZONPOT \\
\hline Permeability & $k_{i}$ & PER & ZONPER & ZONPER \\
\hline Porosity & $\mathrm{n}$ & POR & ZONPOR & ZONPOR \\
\hline
\end{tabular}


The two-phase vater equation of state for "Standard Problem 非" is an example of a user-supplied model. It is presented at the end of this Appendix. Other special material models may be developed after becoming familiar with the functions of the ZON phase PROCESSOR. The chart below lists the subroutines in the ZON phase of the PROCESSOR.

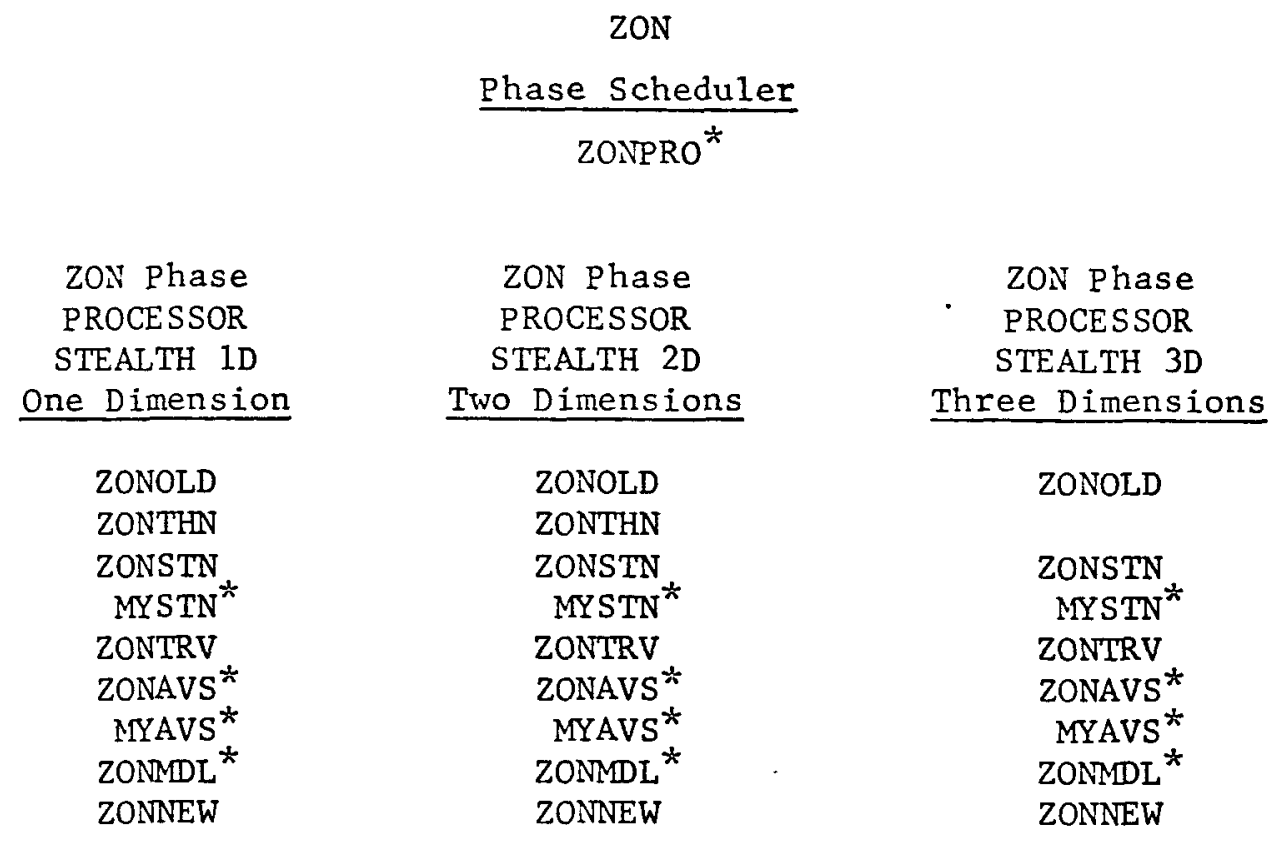

Subroutine ZONPRO schedules the subroutines in the ZON phase PROCESSOR. The logic may be summarized as follows:

ZONOLD transfers last cycle's zone data from arrays in in COMMON /ZONARY/ to local variables in COMMON /ZONVAR/.

\footnotetext{
${ }^{\star}$ Same subroutine used for 1D, 2D, and 3D processors.
} 


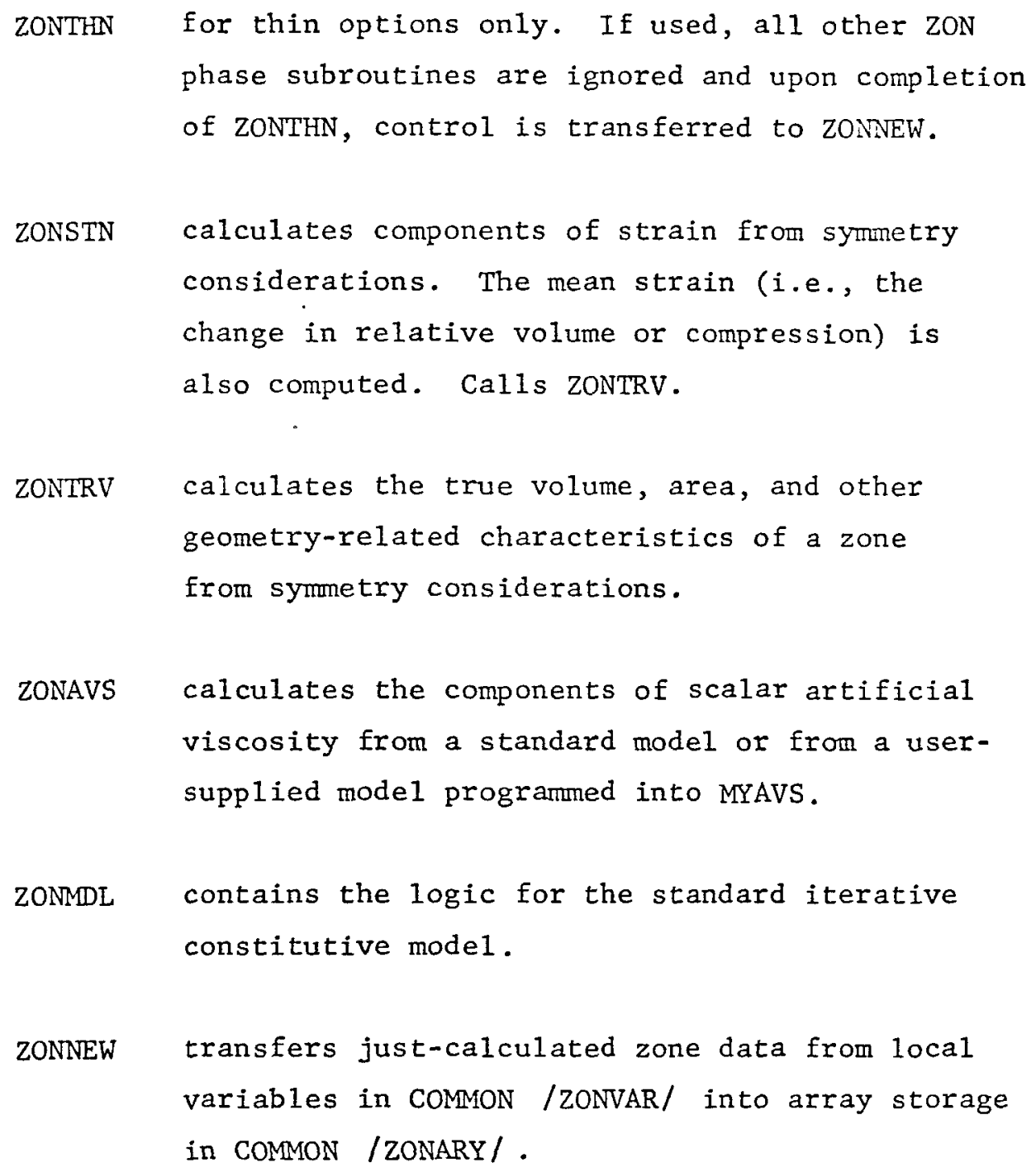

ZONOLD and ZONNEW depend on the contents of the /ZON _-_/ COMMON blocks and, therefore, cannot be changed unless many other subroutines are also changed. ZONML may be completely replaced or changed selectively. In the latter case, changes may be made to ZONMDL logic or to the subroutines 
which ZONMDL calls. Below is a list of all the subroutines called directly or indirectly by subroutine ZONMDL.

\begin{tabular}{|c|c|c|c|}
\hline $\begin{array}{c}\text { Energy } \\
\text { Density } \\
\text { Subroutines } \\
\end{array}$ & $\begin{array}{c}\text { Fluid } \\
\text { Density } \\
\text { Subroutine } \\
\end{array}$ & $\begin{array}{c}\text { Material } \\
\text { Property } \\
\text { Subroutines } \\
\end{array}$ & $\begin{array}{c}\text { Fluid } \\
\text { Material } \\
\text { Property } \\
\text { Subroutines } \\
\end{array}$ \\
\hline $\begin{array}{l}\text { ZONZSE* }^{*} \\
\text { ZONZDE }^{*} \\
\text { ZONZHE }\end{array}$ & ZONZFM & $\begin{array}{c}\text { ZONSTR }^{*} \\
\text { ZONSHR }^{*} \\
\text { MYSHR } \\
\text { ZONSDV } \\
\text { MYSDV }_{*}^{*} \\
\text { ZONYLD }^{*} \\
\text { MYYLD }^{*} \\
\text { ZONSHC }^{*} \\
\text { MYSHC }^{*} \\
\text { ZONTMP }^{*} \\
\text { MYTMP }^{*} \\
\text { ZONCON }^{*} \\
\text { MYCON }^{*} \\
\text { ZONPRH }^{*} \\
\text { MYPRH }^{*} \\
\text { ZONERL }^{*} \\
\text { MYERL }^{*} \\
\text { ZONSPL }^{*} \\
\text { MYSPL }^{*} \\
\text { ZONSSS }^{*} \\
\text { MYSSS }^{*}\end{array}$ & $\begin{array}{l}\text { ZONFPP }^{*} \\
\text { ZONPOT } \\
\text { ZONPER } \\
\text { ZONPOR }^{*}\end{array}$ \\
\hline
\end{tabular}

Most of these subroutines (specifically those under the headings "Material Property Subroutines" and "Fluid Material Property Subroutines") have already been described. The remaining subroutines (ZONZSE, ZONZDE, and ZONZHE) are used to calculate components of the internal energy density. ZONZSE is the source term; ZONZDE is the distortional strain energy; and ZONZHE provides the heat added to a zone. ZONZFM provides the fluid added to a zone.

* Same subroutine used for both processors. 
The subroutine ZONSTR, at the top of the material property colum, is a bit different from the remaining property subroutines. It computes total stress components from the sum of pressure, artificial viscosity, and deviatoric stress components. ZONSTR, as well as ZONZSE, ZONZDE, ZONZHE, ZONPOT, ZONPER, and ZONZFM, use geometry-dependent formulas.

A listing of subroutine ZONMDL is shown below and on the next page.

C
C
C

C

C

C

$\mathrm{C}$

C

C

C

C

C

C

C

C

C

C

C

C

C

C

C

C

C

C

C

C

C

CALCULATE CHANGE IN DISTORTIONAL ENERGY DENSITY AT TIME N+1/2 FROM STRESS DEVIATORS AT TIME N

SXXN $=$ SXXO

SYYN $\quad=$ SYYO

$S Z Z N=S Z Z O$

SXYN $\quad=$ SXYO

CALI ZONZDE

CALCULATE CHANGE IN HEAT ENERGY DENSITY AT TIME $\mathrm{N}+1 / 2$

FROM HEAT FLUXES AT TIME $\mathrm{N}+1 / 2$

CALCULATED FROM TEMPERATURES AT TIME N

CALL ZONZHE

CALCULATE SOURCE ENERGY DENSITY TO BE DEPOSITED AT TIME N

CALI ZONZSE

CALCULATE CHANGE IN INTERNAL ENERGY DENSITY AT TIME $\mathrm{N}+1 / 2$

FROM PRESSURE AT TIME $N$, ARTIFICIAL VISCOSITY AT TIME $N+1 / 2$, CHANGE OF RELATIVE VOLUME AT TIME $\mathrm{N}+1 / 2$ AND CHANGES IN ENERGY DENSITY AS DEFINED ABOVE

ZIEN = ZIEO - (PRHO + AVSH) * DLRLVH + DLZDEH + DLZHEH + ZSEO

CALCULATE TEMPERATURE AT TIME $\mathrm{N}+1$

CALI ZONTMP

CALL ZONPOR

CALCULATE ZONE POROSITY AT TIME N+1

CATH ZONPOR

CALL ZONZFM

CALCULATE FLUID PORE PRESSURE AT TIME N+1

CALL ZONFPP

CALCULATE PRESSURE HEAD, GRAVITY HEAD AND TOTAL POTENTIAL AT TIME N+1

CALL ZONPOT

CALCULATE EFFECTIVE PRESSURE AT TIME $\mathrm{N}+1$

CALL ZONPRH 
C

C

C

C CALCULATE SHEAR MODULUS AT TIME $\mathrm{N}+1$

CALL ZONSHR

C

C

C

$\mathrm{C}$

C

C

C

C

C

C

C

C

C

C

C

C

CALCULATE STRESS DEVIATORS AT TIME $\mathrm{N}+1$

CALL ZONSDV

CALCULATE TOTAL STRESS AT TIME N+1

CALL ZONSTR

CALCULATE ENERGY RELEASE PRESSURE CONTRIBUTION AT TIME $\mathrm{N}+1$

CALL ZONERL

CALCULATE SPALL STRESS AT TIME $\mathrm{N}+1$

CALL ZONSPL

CALCULATE SOUND SPEED SQUARED AT TIME N+1

CALL ZONSSS

CALCULATE MATRIX PERMEABILITY AT TIME N+1

CALL ZONPER

CALCULATE SPECIFIC HEAT CAPACITY AT TIME N+1

CALL ZONSHC

CALCULATE CONDUCTIVITY AT TIME $\mathrm{N}+1$

GALL ZONCON 
THERMODYNAMIC MODEL

The thermodynamic model is a relationship between scalar properties of a material. Certain sets of properties (e.g., pressure (p), internal energy density $(u)$, and relative volume (V)) form what is called a complete equation of state because mechanical, thermal, and caloric properties are included. The purpose of the pressure equation of state is to compute pressure from internal energy and relative volume. (Use of relative volume requires that a reference density, $\rho^{\circ}$, be defined; $V \equiv \rho^{\circ} / \rho$ where $f$ is the density.)

\section{Pressure Equation of State}

Correspondence between FORTRAN input names and scientific notation is shown below.

$$
\begin{aligned}
& \text { MEOS }=\text { equation of state, model-type value } \\
& \text { EOSO = equation-of-state parameter, } b_{0} \\
& \text { EOS1 }=\text { equation-of-state parameter, } b_{1} \\
& \text { EOS2 }=\text { equation-of-state parameter, } b_{2} \\
& \text { EOS3 }=\text { equation-of-state parameter, } b_{3} \\
& \text { EOS4 }=\text { equation-of-state parameter, } b_{4} \\
& \text { EOS5 }=\text { equation-of-state parameter, } b_{5} \\
& \text { EOS6 }=\text { equation-of-state parameter, } b_{6} \\
& \text { EOS7 }=\text { equation-of-state parameter, } b_{7} \\
& \text { EOS8 }=\text { equation-of-state parameter, } b_{9} \\
& \text { EOS9 }=\text { equation-of-state parameter, } b_{9} \\
& \text { MEOS } 1.0 \\
& \text { Pressure is a function of compression }(\mu) \text { where } \mu \equiv \frac{1}{V}-1 . \\
& \quad \text { p }=f\left(b_{1}, b_{2}, b_{3}, b_{4}, \mu\right)
\end{aligned}
$$

The functional form $f$ is the standard function type given by the value bo (see Appendix "Functions"). For example, if $b_{0}=1.0$, then the pressure equation of state is

$$
p=b_{1}+b_{2} \mu+b_{3} \mu^{2}+b_{4} \mu^{3}
$$


MEOS $=2.0$

Pressure is a function of internal energy density (u) where $\mathrm{u} \equiv \hat{\mathrm{u}}_{\mathrm{o}}{ }^{\circ}$.

$$
\mathrm{p}=f\left(\mathrm{~b}_{1}, \mathrm{~b}_{2}, \mathrm{~b}_{3}, \mathrm{~b}_{4}, \mathrm{u}\right) \quad \mathrm{b}_{5} \leq \mathrm{u} \leq \mathrm{b}_{6}
$$

The functional form $f$ is the standard function type given by the value $b_{\circ}$ (see Appendix "Functions"). For example, if $b_{0}=3.0$, then the pressure equation of state is

$$
p=\left(b_{1}+b_{2} u+b_{3} u^{2}\right) e^{-b_{4} u}
$$

MEOS $=3.0$

Pressure is a function of internal energy density and compression.

$$
p=f_{\ell}\left(b_{1}, b_{2}, b_{3}, b_{4}, \mu\right)+u\left[f_{m}\left(b_{8}, b_{9}, \mu\right)\right] \quad b_{5} \leq \mu \leq b_{6}
$$

The functional forms $f_{\ell}$ and $f_{m}$ are the standard function types* given by the values $b_{0}$ and $b_{7}$, respectively (see Appendix "Functions"). For example, if $b_{0}=5.0$, and $b_{7}=2.0$, then the pressure equation of state is

$$
p=b_{1} e^{\left(b_{2} e^{b_{3} \mu}\right)}+b_{4}+u\left(b_{8}+b_{9} \mu\right)
$$

For $f_{a}$, the coefficients $b_{a}$ and $b_{9}$ correspond to the first two function coefficients, $a_{1}$ and $a_{2}$. The other two coefficients, $a_{3}$ and $a_{4}$, are assumed to be zero.

\footnotetext{
${ }^{\star} \ell$ and $m$ designate function types $1-10$.
} 


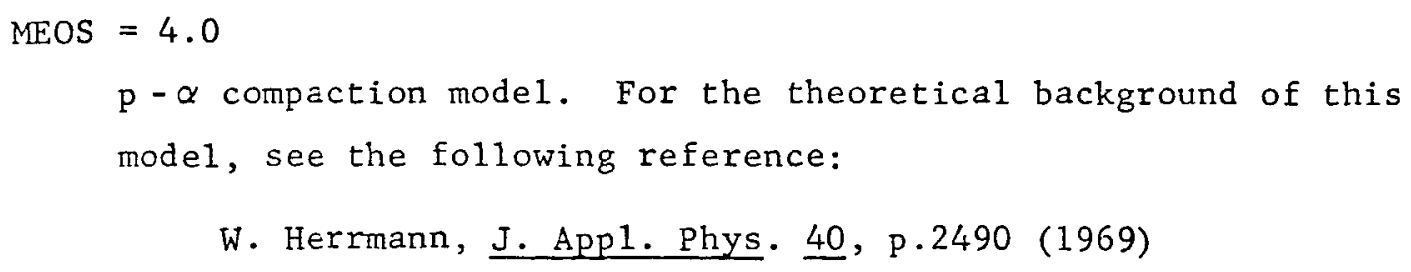

Pressure is a function of relative volume (V) and internal energy density.

$$
p=b_{2}\left(1.0-\frac{b_{4}}{b_{2} V}\right) e^{-b_{2} V}+b_{1}\left(1.0-\frac{b_{4}}{b_{3} V}\right) e^{-b_{3} V}+b_{4} \frac{u}{V}
$$

MEOS $=6.0,7.0,8.0,9.0,10.0$

User-supplied, pressure equation-of-state models. Model logic is programed into subroutine MYPRH. An example of MYPRH is presented at the end of this Appendix. 
FLUID THERMODYNAMIC MODEL

The fluid thermodynamic model is a relationship between scalar properties of a material. Certain sets of properties (e.g., pressure $\left(p_{f}\right)$, relative fluid mass $(M)$, temperature $(T)$ ) form what is called a complete equation of state because mechanical, thermal, and hydraulic properties are included. The purpose of the pressure equation of state is to compute pressure from relative fluidmass and temperature. (Use of relative fluid mass requires that a reference fluid density, $\rho_{f}^{0}$, be defined; $M \equiv \rho_{f} / \rho_{f}^{o}$ where $\rho_{f}$ is the density.)

\section{Fluid Pressure Equation of State}

Correspondence between FORTRAN input names and scientific notation is shown below.

$$
\begin{aligned}
& \text { MFPP }=\text { fluid equation of state, model-type value } \\
& \text { FPPO }=\text { fluid equation-of-state parameter, } b_{0} \\
& \text { FPP1 }=\text { fluid equation-of-state parameter, } b_{1} \\
& \text { FPP2 }=\text { fluid equation-of-state parameter, } b_{2} \\
& \text { FPP3 }=\text { fluid equation-of-state parameter, } b_{3} \\
& \text { FPP4 }=\text { fluid equation-of-state parameter, } b_{4} \\
& \text { MFPP = } 1.0 \\
& \text { Pore pressure is an incremental function of relative fluid } \\
& \text { mass and temperature. } \\
& \Delta_{p_{f}}=b_{2}(\Delta M / M)+b_{2} b_{3} \Delta T \\
& b_{z}=\text { fluid bulk modulus } \\
& b_{3}=\text { fluid volumetric thermal expansion coefficient } \\
& b_{4}=\text { fluid dynamic viscosity }
\end{aligned}
$$


STRENG TH MODEL

The standard strength model is made up of a yield stress ( $\mathrm{Y}$ ) model, a shear modulus $(G)$ model, and a spall strength $\left(P_{\min }\right)$ model. Each model contributes to the strength calculation in a different way.

Yield Stress Mode1

Correspondence between FORTRAN input names and scientific notation is shown below.

$$
\begin{aligned}
& \text { MYLD }=\text { yield stress model-type value } \\
& \text { YLDO }=\text { yield stress parameter, } y_{0} \\
& \text { YLD1 }=\text { yield stress parameter, } y_{1} \\
& \text { YLD2 }=y \text { yeld stress parameter, } y_{2} \\
& \text { YLD3 }=y \text { yield stress parameter, } y_{3} \\
& \text { YLD4 }=y \text { yeld stress parameter, } y_{4}
\end{aligned}
$$

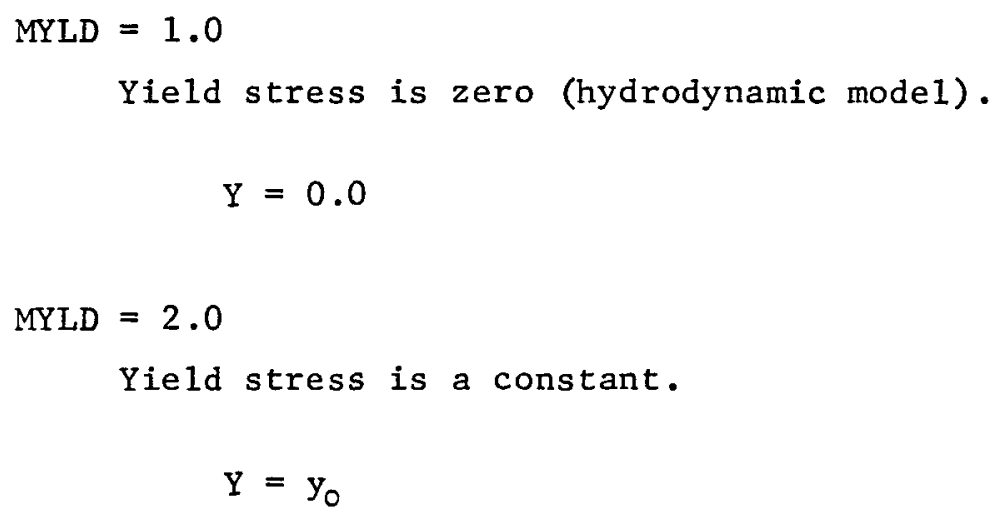


MYLD = 3.0

Yield stress is a function of pressure (p) only.

$$
\mathrm{Y}=f\left(\mathrm{y}_{1}, \mathrm{y}_{2}, \mathrm{y}_{3}, \mathrm{y}_{4}, \mathrm{p}\right)
$$

The functional form $f$ is the standard function type given by the value $y_{0}$ (see Appendix "Functions"). For example, if $\mathrm{y}_{0}=2.0$, then the yield stress model is

$$
Y=\frac{y_{1}+y_{2} p}{1+y_{3} p+y_{4} p^{2}}
$$

MYLD $=4.0$

Yield stress is a function of internal energy density (u) only.

$$
\mathrm{Y}=f\left(\mathrm{y}_{1}, \mathrm{y}_{2}, \mathrm{y}_{3}, \mathrm{y}_{4}, \mathrm{u}\right)
$$

The functional form $f$ is the standard function type given by the value $y_{0}$ (see Appendix "Functions"). For example, if $\mathrm{y}_{0}=4.0$, then the yield stress model is

$$
Y=y_{1}+y_{2} e^{\left(y_{3} u^{2}+y_{4} u\right)}
$$

MYLD $=5.0$

Yield stress is a function of the absolute value of distortional energy density $(|z|)$ only.

$$
\mathrm{Y}=f\left(\mathrm{y}_{1}, \mathrm{y}_{2}, \mathrm{y}_{3}, \mathrm{y}_{4},|\mathrm{z}|\right)
$$

The functional form $f$ is the standard function type given by the value $y_{0}$ (see Appendix "Functions"). For example, if $y_{0}=1.0$, then the yield stress model is

$$
Y=y_{1}+y_{2}|z|+y_{3}|z|^{2}+y_{4}|z|^{3}
$$




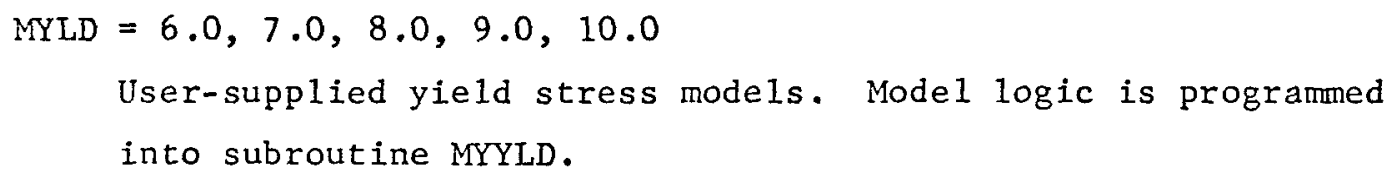


Shear Modulus Mode1

Correspondence between FORTRAN input names and scientific notation is shown below. $(G \geq 0$.)

$$
\begin{aligned}
& \text { MSHR = shear modulus mode1-type value } \\
& \text { SHRO = shear modulus parameter, } g_{0} \\
& \text { SHR1 = shear modulus parameter, } g_{1} \\
& \text { SHR2 = shear modulus parameter, } g_{2} \\
& \text { SHR3 = shear modulus parameter, } g_{3} \\
& \text { SHR4 = shear modulus parameter, } g_{4}
\end{aligned}
$$

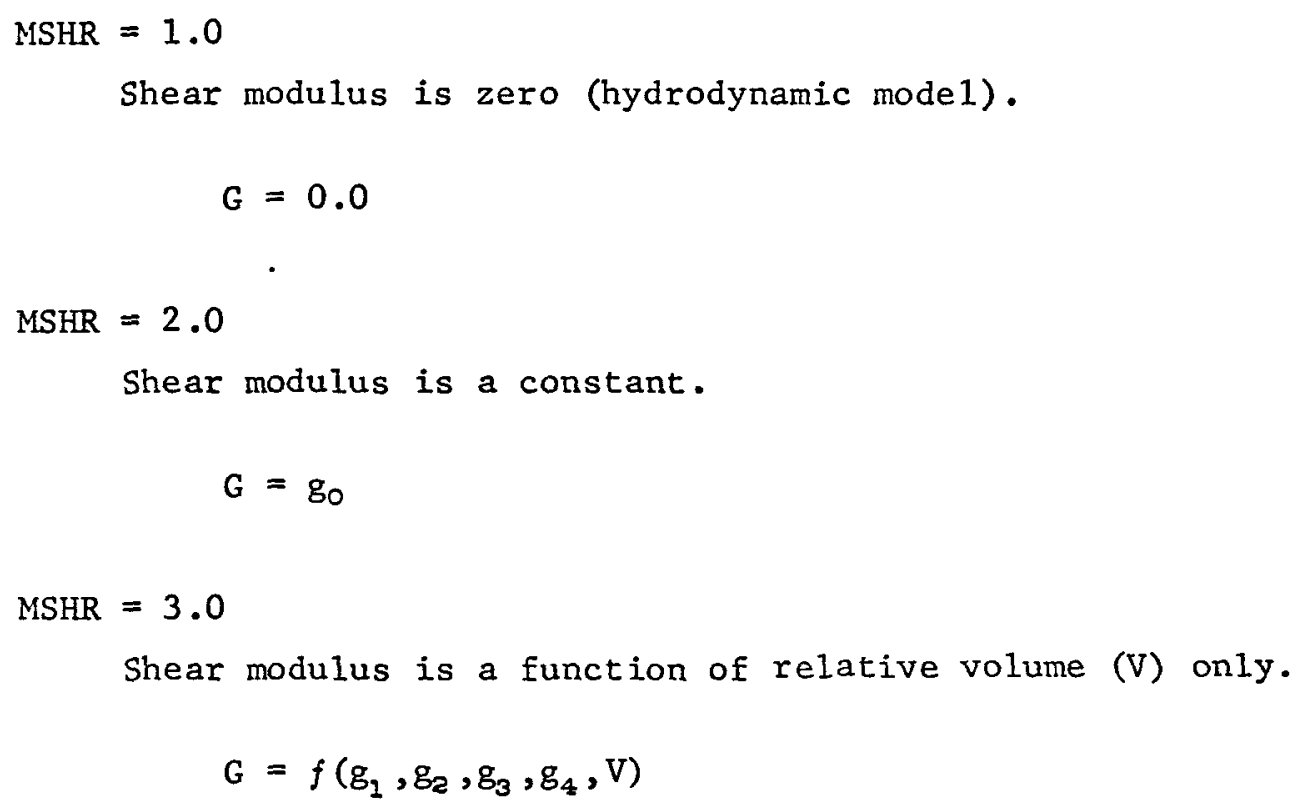

The functional form $f$ is the standard function type given by the value go (see Appendix "Functions"). For example, if $g_{0}=2.0$, then the shear modulus model is

$$
G=\frac{g_{1}+g_{2} V}{1+g_{3} V+g_{4} V^{2}}
$$


MSHR $=4.0$

Shear modulus is a function of internal energy density (u) only.

$$
G=f\left(g_{1}, g_{2}, g_{3}, g_{4}, u\right)
$$

The functional form $f$ is the standard function type given by the value go (see Appendix "Functions"). For example, if $g_{0}=4.0$, then the shear modulus model is

$$
G=g_{1}+g_{2} e^{\left(g_{3} u^{2}+g_{4} u\right)}
$$

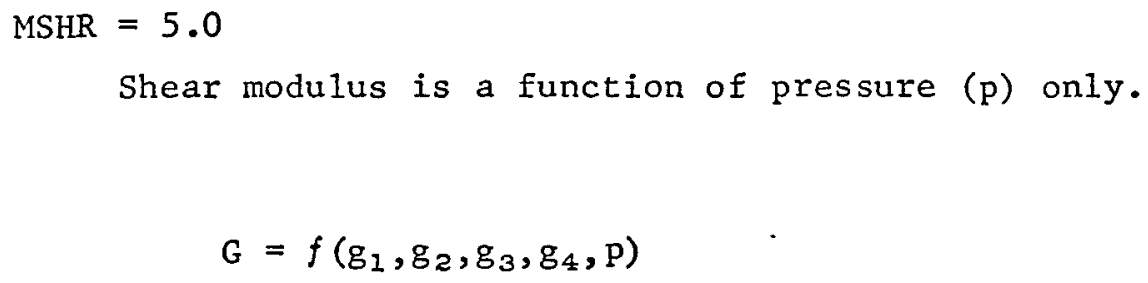

The functional form $f$ is the standard function type given by the value go (see Appendix "Functions"). For example, if $\mathrm{go}_{0}=1.0$, then the shear modulus model is

$$
G=g_{1}+g_{2} p+g_{3} p^{2}+g_{4} p^{3}
$$

MSHR $=6.0,7.0,8.0,9.0,10.0$

User-supplied shear modulus models. Model logic is programmed in subroutine MYSHR. 
Spal1 Mode1

Correspondence between FORTRAN input names and scientific notation is shown below.

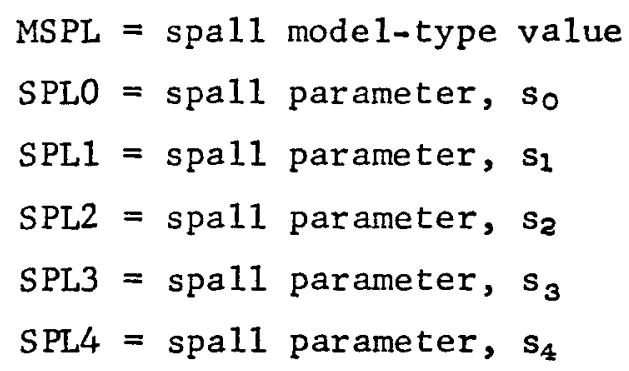

Spal1 is zero (hydrodynamic mode1).

$$
\mathrm{P}_{\text {min }}=0.0
$$

MSPL $=2.0$

Spall is a negative constant.

$$
\mathrm{p}_{\text {min }}=\mathrm{s}_{\mathrm{o}}
$$

MSPL $=3.0$

Spall is a function of relative volume (V) only.

$$
P_{\min }=f\left(s_{1}, s_{2}, s_{3}, s_{4}, v\right)
$$

The functional form $f$ is the standard function type given by the value so (see Appendix "Functions"). For example, if $s_{0}=2.0$, then the spall model is

$$
p_{\min }=\frac{s_{1}+s_{2} V}{1+s_{3} V+s_{4} v^{2}}
$$


MSPL $=4.0$

Spa11 is a function of internal energy density (u) only.

$$
\mathrm{P}_{\min }=f\left(\mathrm{~s}_{1}, \mathrm{~s}_{2}, \mathrm{~s}_{3}, \mathrm{~s}_{4}, \mathrm{u}\right)
$$

The functional form $f$ is the standard function type given by the value $s_{\circ}$ (see Appendix "Functions"). For example, if $s_{0}=4.0$, then the spall model is

$$
p_{\min }=s_{1}+s_{2} e^{\left(s_{3} u^{2}+s_{4} u\right)}
$$

MSPL $=5.0$

Spall is a function of the absolute value of distortional energy density $(|z|)$ only.

$$
\mathrm{p}_{\min }=f\left(\mathrm{~s}_{1}, \mathrm{~s}_{2}, \mathrm{~s}_{3}, \mathrm{~s}_{4},|\mathrm{z}|\right)
$$

The functional form $f$ is the standard function type given by the value so (see Appendix "Functions"). For example, if $s_{0}=1.0$, then the spall model is

$$
p_{\min }=s_{1}+s_{2}|z|+s_{3}|z|^{2}+s_{4}|z|^{3}
$$

MSPL $=6.0,7.0,8.0,9.0,10.0$

User-supplied spal1 models. Model logic is programmed into subroutine MYSPL. 
ENERGY RELEASE MODEL

The energy release model is a relationship between the chemically evolved energy density (E) of a material and the chemical components of the material.

Correspondence between FORTRAN input names and scientific notation is shown below.

$$
\begin{aligned}
& \text { MERL = energy release model-type value } \\
& \text { ERLO = energy release parameter, } r_{0} \\
& \text { ERL1 = energy release parameter, } r_{1} \\
& \text { ERL2 = energy release parameter, } r_{2} \\
& \text { ERL3 = energy release parameter, } r_{3} \\
& \text { ERL4 = energy release parameter, } r_{4}
\end{aligned}
$$

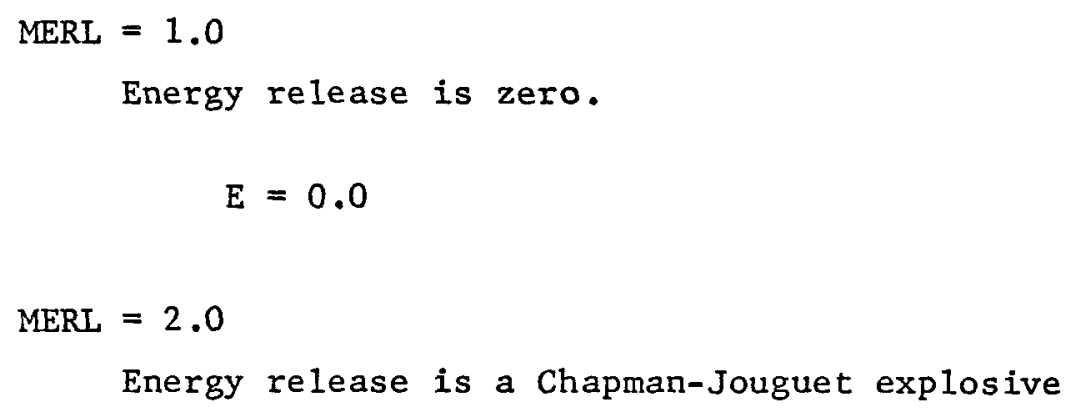


HEAT FLOW MODEL

The heat flow model is made up of a conductivity ( $k$ ) model and a specific heat capacity $\left(C_{V}\right)$ mode1. Each model contributes to the heat flow calculation in a different way.

\section{Conductivity Model}

Correspondence between FORTRAN input names and scientific notation is shown below. $(k \geq 0$.)

$$
\begin{aligned}
& \text { MCON = conductivity model-type value } \\
& \text { CONO = conductivity parameter, } k_{0} \\
& \text { CON1 = conductivity parameter, } k_{1} \\
& \text { CON2 = conductivity parameter, } k_{2} \\
& \text { CON3 = conductivity parameter, } k_{3} \\
& \text { CON4 = conductivity parameter, } k_{4}
\end{aligned}
$$

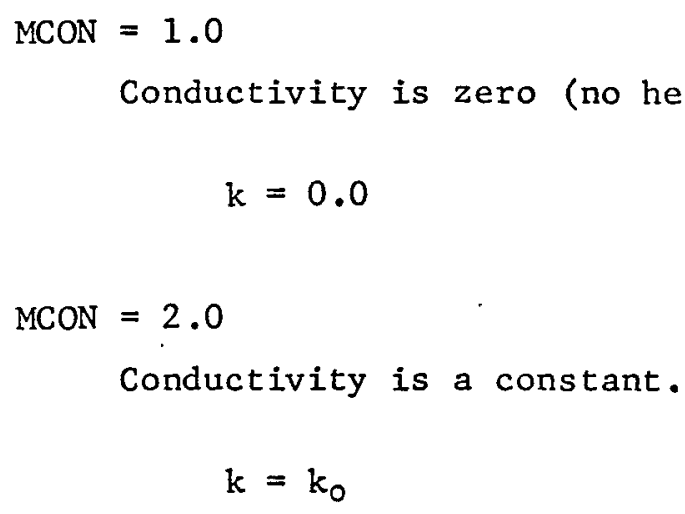


$\mathrm{MCON}=3.0$

Conductivity is a function of temperature $(T)$ only.

$$
k=f\left(k_{1}, k_{2}, k_{3}, k_{4}, T\right)
$$

. The functional form $f$ is the standard function given by the value $k_{0}$ (see Appendix "Functions"). For example, if

$k_{0}=2.0$, then the conductivity model is

$$
k=\frac{k_{1}+k_{2} T}{1+k_{3} T+k_{4} T^{2}}
$$

$\mathrm{MCON}=4.0,5.0$

No models presently defined.

MCON $=6.0,7.0,8.0,9.0,10.0$

User-supplied, conductivity models. Model logic is programmed into MYCON. 


\section{Specific Heat Capacity Mode1}

Correspondence between FORTRAN input names and scientific notation is shown below. $\left(C_{V} \geq 0.\right)$

$$
\begin{aligned}
& \text { MSHC = specific heat capacity model-type value } \\
& \text { SHCO = specific heat capacity parameter, } c_{0} \\
& \text { SHC1 }=\text { specific heat capacity parameter, } c_{1} \\
& \text { SHC2 }=\text { specific heat capacity parameter, } c_{2} \\
& \text { SHC3 }=\text { specific heat capacity parameter, } c_{3} \\
& \text { SHC4 }=\text { specific heat capacity parameter, } c_{4}
\end{aligned}
$$

$\mathrm{MSHC}=1.0$

Specific heat capacity is zero.

$$
c_{V}=0.0
$$

$\mathrm{MSHC}=2.0$

Specific heat capacity is a constant.

$$
c_{V}=c_{0}
$$

For hydrological problems (i.e., NSDTS $=5,6$, or 7), the fluid specific heat capacity is a constant

$$
c_{v_{f}}=c_{i}
$$


MSHC $=3.0$

Specific heat capacity is a function of temperature $(T)$ only.

$$
T=f\left(c_{1}, c_{2}, c_{3}, c_{4}, T\right)
$$

The functional form $f$ is the standard function given by the value $c_{0}$ (See Appendix "Functions"). For example, if $c_{0}=2.0$, then the specific heat capacity model is

$$
c_{V}=\frac{c_{1}+c_{2} T}{1+c_{3} T+c_{4} T^{2}}
$$

$$
\text { MSHC }=4.0,5.0
$$

No models presently defined.

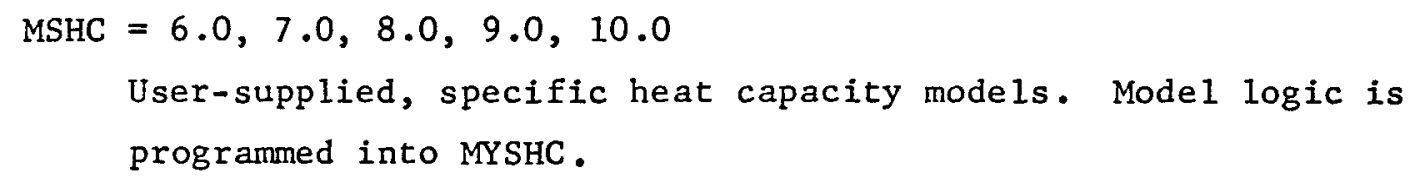


FLUID FLOW MODEL

The fluid flow model is made up of a permeability (k) model and a porosity $(n)$ model. Each model contributes to the fluid flow calculation in a different way.

Permeability Model

Correspondence between FORTRAN input names and scientific notation is shown below. $(k \geq 0$.)

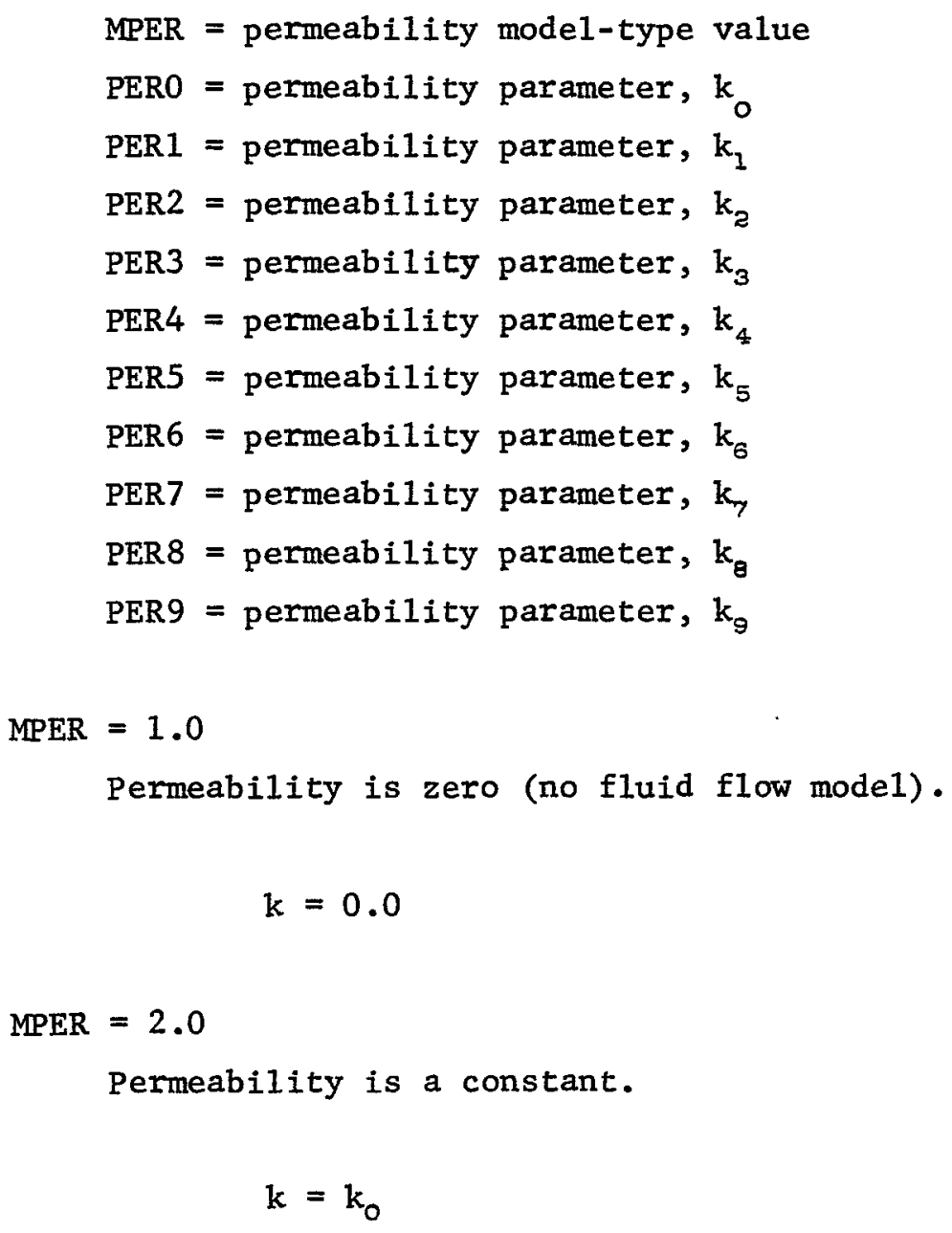


MPER $=3.0$

Permeability is an anisotropic constant

$$
\begin{aligned}
& k_{x}=k_{0} \\
& k_{y}=k_{5}
\end{aligned}
$$

MPER $=4.0$

Permeability is a function of porosity $(\bar{n})$ where $\bar{n}=n / n^{0}$.

$$
\begin{aligned}
& k_{x}=f\left(k_{1}, k_{2}, k_{3}, k_{4}, n\right) \\
& k_{y}=f\left(k_{6}, k_{7}, k_{8}, k_{9}, n\right)
\end{aligned}
$$

The functional form $f$ is the standard function given by the value $k_{0}$ for $k_{x}$ and $k_{5}$ for $k_{y}$ (see Appendix "Functions"). For example, if $k_{0}=k_{5}=1.0$, then the permeability model is

$$
\begin{aligned}
& k_{x}=k_{1}+k_{2} \bar{n}+k_{3} \bar{n}^{2}+k_{4} \bar{n}^{3} \\
& k_{y}=k_{5}+k_{6} \bar{n}+k_{7} \bar{n}^{2}+k_{9} \bar{n}^{3}
\end{aligned}
$$

$$
\text { MPER }=5.0
$$

No model presently defined.

MPER $=6.0,7.0,8.0,9.0,10.0$

User-supplied, permeability models. Model logic is programmed into MYCON. 
Porosity Model

Correspondence between FORTRAN input names and scientific notation is shown below. $(0 \leq n \leq 1)$

$$
\begin{aligned}
& \text { MPOR = porosity model type value } \\
& \text { PORO = porosity parameter, } n_{0} \\
& \text { POR1 = porosity parameter, } n_{1} \\
& \text { POR2 = porosity parameter, } n_{2} \\
& \text { POR3 = porosity parameter, } n_{3} \\
& \text { POR4 = porosity parameter, } n_{4} \\
& \text { POR5 = porosity parameter, } n_{5} \\
& \text { POR6 = porosity parameter, } n_{6} \\
& \text { POR7 = porosity parameter, } n_{7} \\
& \text { POR8 = porosity parameter, } \mathrm{n}_{8} \\
& \text { POR9 = porosity parameter, } n_{9} \\
& \mathrm{MPOR}=1.0 \\
& \text { Porosity is zero (no fluid model) } \\
& \mathrm{n}=0.0 \\
& \text { MPOR }=2.0 \\
& \text { Porosity is a constant } \\
& \mathrm{n}=\mathrm{n}_{\mathrm{o}}
\end{aligned}
$$




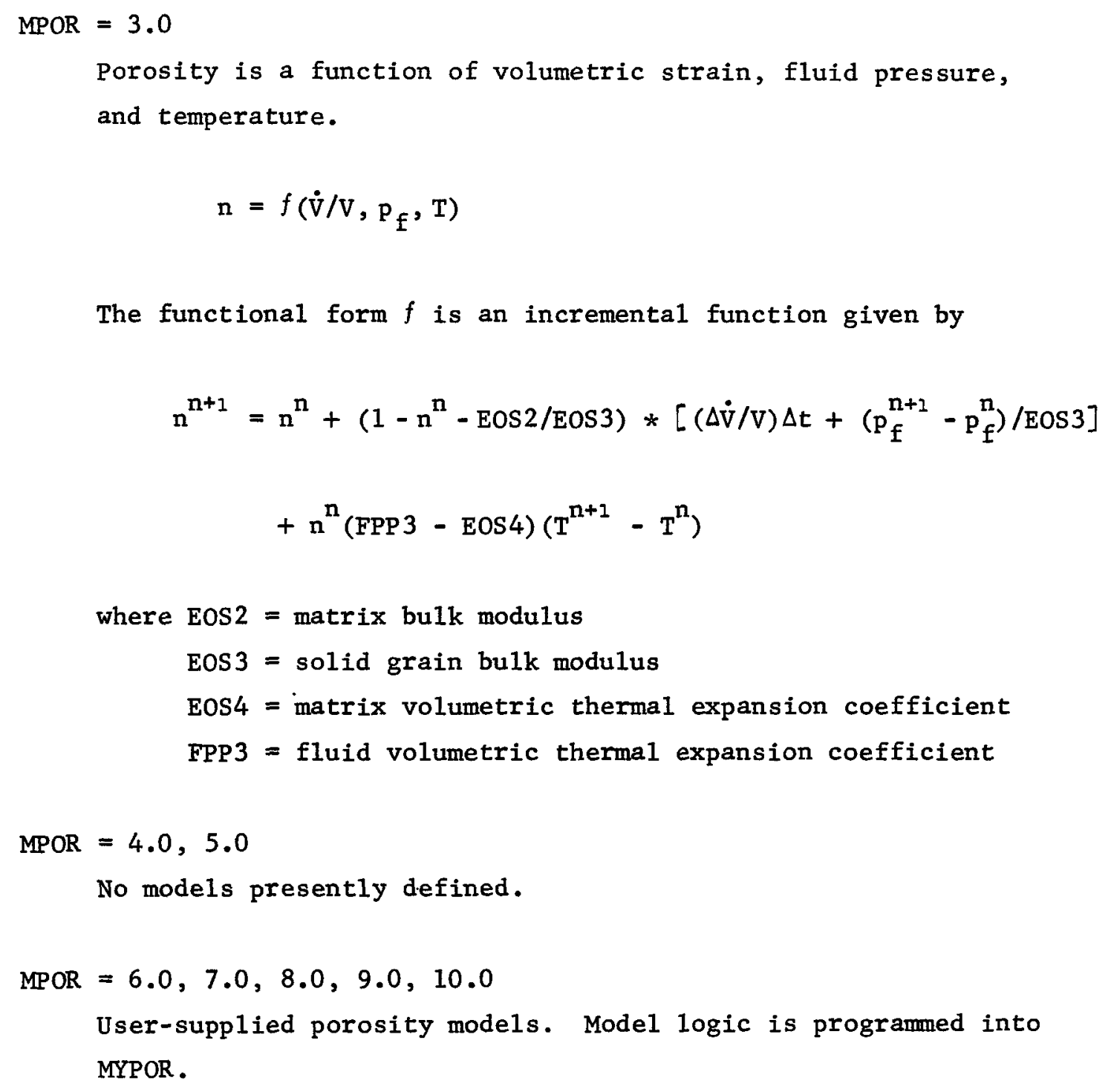

The functional form $f$ is an incremental function given by

$$
\begin{aligned}
n^{n+1}= & n^{n}+\left(1-n^{n}-\operatorname{EOS} 2 / \operatorname{EOS} 3\right) *\left[(\Delta \dot{V} / V) \Delta t+\left(p_{f}^{n+1}-p_{f}^{n}\right) / E O S 3\right] \\
& +n^{n}(\operatorname{FPP} 3-\operatorname{EOS} 4)\left(T^{n+1}-T^{n}\right)
\end{aligned}
$$

where $\mathrm{EOS} 2$ = matrix bulk modulus

EOS 3 = solid grain bulk modulus

EOS4 = matrix volumetric thermal expansion coefficient

FPP3 = fluid volumetric thermal expansion coefficient

$$
\mathrm{MPOR}=4.0,5.0
$$

No models presently defined.

$\mathrm{MPOR}=6.0,7.0,8.0,9.0,10.0$

User-supplied porosity models. Model logic is programmed into MYPOR . 
EXAMPLE OF USER-SUPPLIED MODEL

SUBROUTINE MYPRH

c

C

C

C

C

C

C

c

c

e

c

c

C

c

c

c

C

-CALL zONVAR

* CALL PRBVAR

- CALL TIHVAR

c

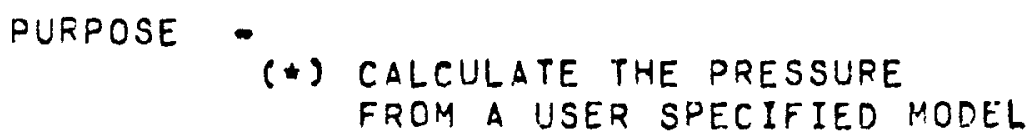

DATA BMOD/19575.6/

DATA NSW /2/ 
TSAT IS THE SATURATION TEMPERATURE (CELCIUS)

PSAT IS THE SATURATION PRESSURE (BARS)

VSATL IS THE SPECIFIC VOL.UME OF SATURATED LIOIJID (CC/GM)

VSATV IS THE SPECIFIC VOLUME OF SATURATED VAPOR (CC/GM)

ESATL IS THE ENTHALPH OF SATURATED LIOUID ( $J / G M$ )

ESATV IS THE ENTHALPY OF SATURATED VAPOR ( $J / G M)$

DATA (TSAT(L),L $=1,44) \quad 10,0,001,10,, 20,30,040,50,60,170,080$, $190,1100,1110,1120,130,140,150,160,170,180,1190,1200, .218 \ldots$

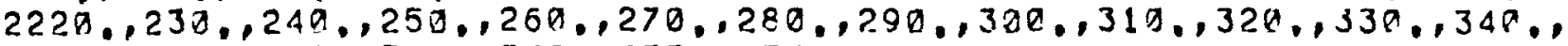
$3350,, 360, \ldots 379,, 371,372,373, \ldots 374,, 374,15 /$

DATA (PSAT(L),L=1,44) $1.006108, .806112, .012271, .023368, .042418$, $1.07375, .12335, .19919, .31161, .47358, .70199,1.01325,1.4327,1.9854$, $22.7011,3,6136,4.7597,6.1894,7.9292,10.027,12,553,15.55,19.08$, $323,282,27.979,33.48,39.776,46.941,55.052,64,191,74.449,85.917$, $493,694,112,89,128,64,146,08,165,37,186,74,218.53,213,06,215,63$, $5218,2,220,9,221.21$

DATA (VSATL(L),L $=1,44) / 1,00621,1.0202101,1.0004,1.0018,1,0044$, $11.0079,1.0121,1.0171,1.0228,1.029,1,0359,1,0435,1.0515,1.0603$, $21.0697,1.0798,1.0906,1.1021,1,1144,1.1275,1,1415,1,1565,1.1726$. $31,19,1.2087,1.2291,1,2512,1.2755,1.3023,1.3321,1.3655,1.4036$, $41.4475,1,4992,1.562,1.639,1.741,1,894,2.22,2.29,2.38,2.51,2.8$, 53.171

DATA (VSATV(L),L=1,44) /206288,,206146,,106422,,57836,,32929,. $119546,12045,17677,6,5045,3,3408,3,2360.9,1673,1210,1,891.71$, $2668,32,508,66,392,57,306,85,242,62,193,85,156,35,127,19,104,265$, $386,062,71.472,59,674,50.056,42.149,35,599,39,133,25.537,21.643$, $418,316,15,451,12,967,10.779,8,805,6,943,4,93,4,68,4,4,4,05,3.47$, $53.17 /$

DATA (ESATL(L),L=1,44) $1-.0416, .080611,41,99,83,86,125,66,167.47$, $1209,3,251,1,293, .334,9,376,9,419,1,461,3,503,7,546,3,589,1,632,2$, $2675,5,719,1,763,1,807,5,852,4,897,7,943,7,990,3,1037,6,1085,8$, $31135,11135.2,1236.8,1290, .1345, .1402, .1462,1526, .1596,1672 \ldots$ $41762,11892,11913,1937,11969,2032,2095.1$

DATA (ESATV(L),L $=1,44) / 2501,, 2501,, 2519, .2538,, 2556, .2574, .2592 \ldots$ $12609,2626 \ldots 2643 \ldots, 2660, .2676 \ldots, 2691 \ldots, 2706, .2720, .2734 \ldots, 2747 \ldots 2758 \ldots$ $22769, .2778 \ldots, 2786, .2793 \ldots 2798, .2802, .2803 \ldots, 2803 \ldots 2801, .2796 \ldots 2790 \ldots$ $32780 \ldots 2766, .2749 \ldots 2727 \ldots 2700, .2666 \ldots 2623 \ldots, 2565 \ldots 2481 \ldots 2331 ., 2305 \ldots$ $42273, .2230, .2146, .2095 .1$ 


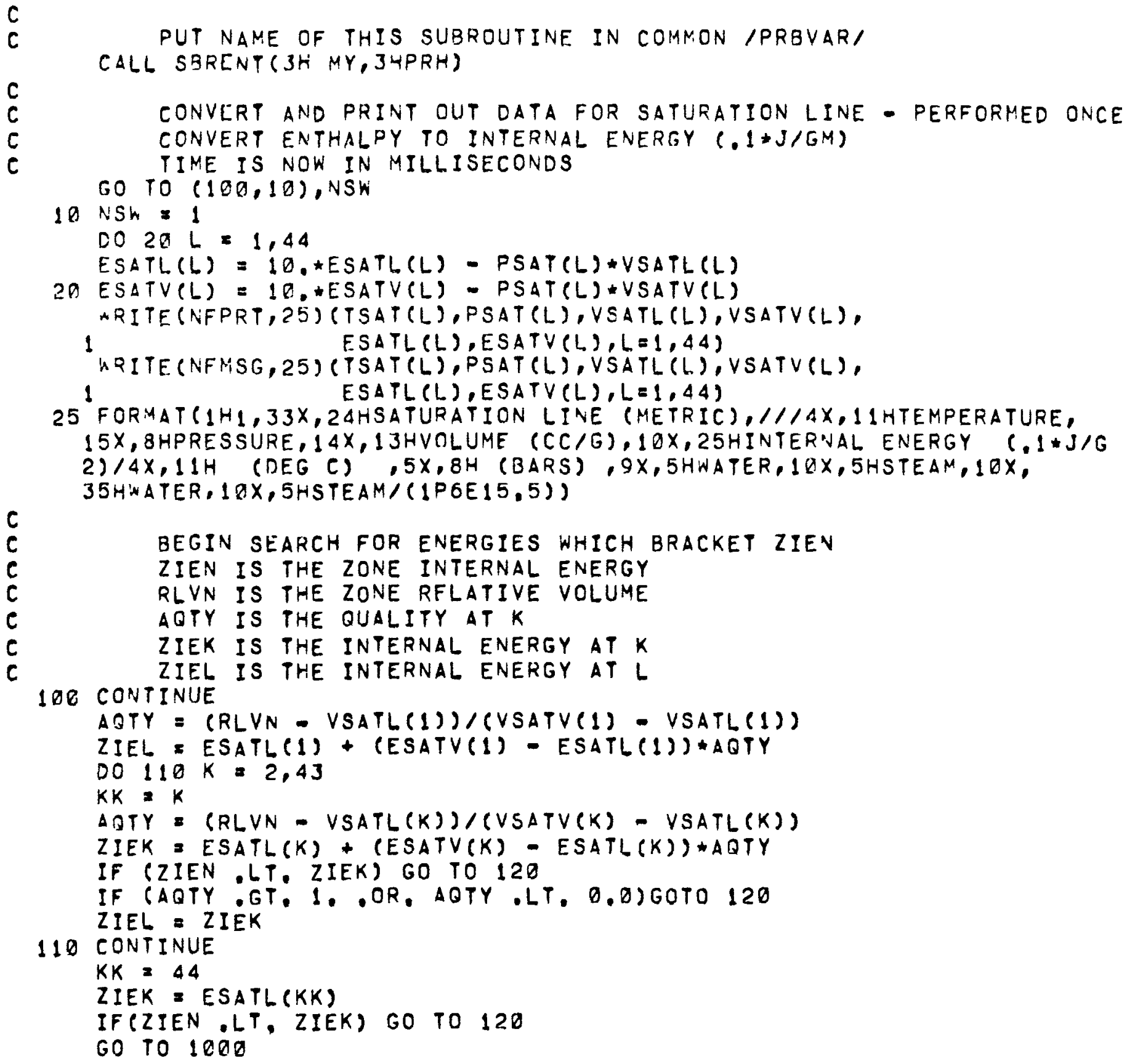


SUBROUTINE MYSSS

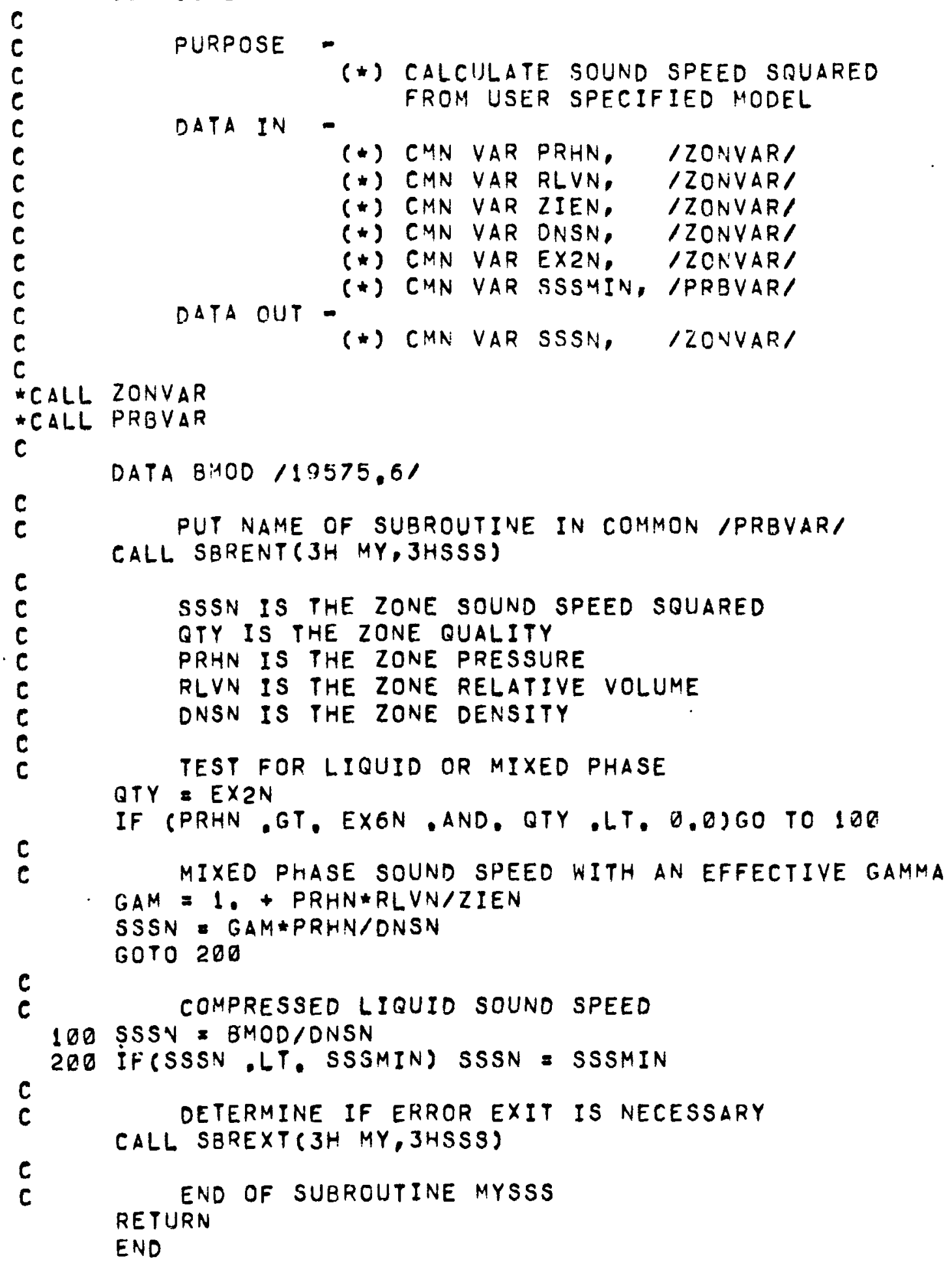




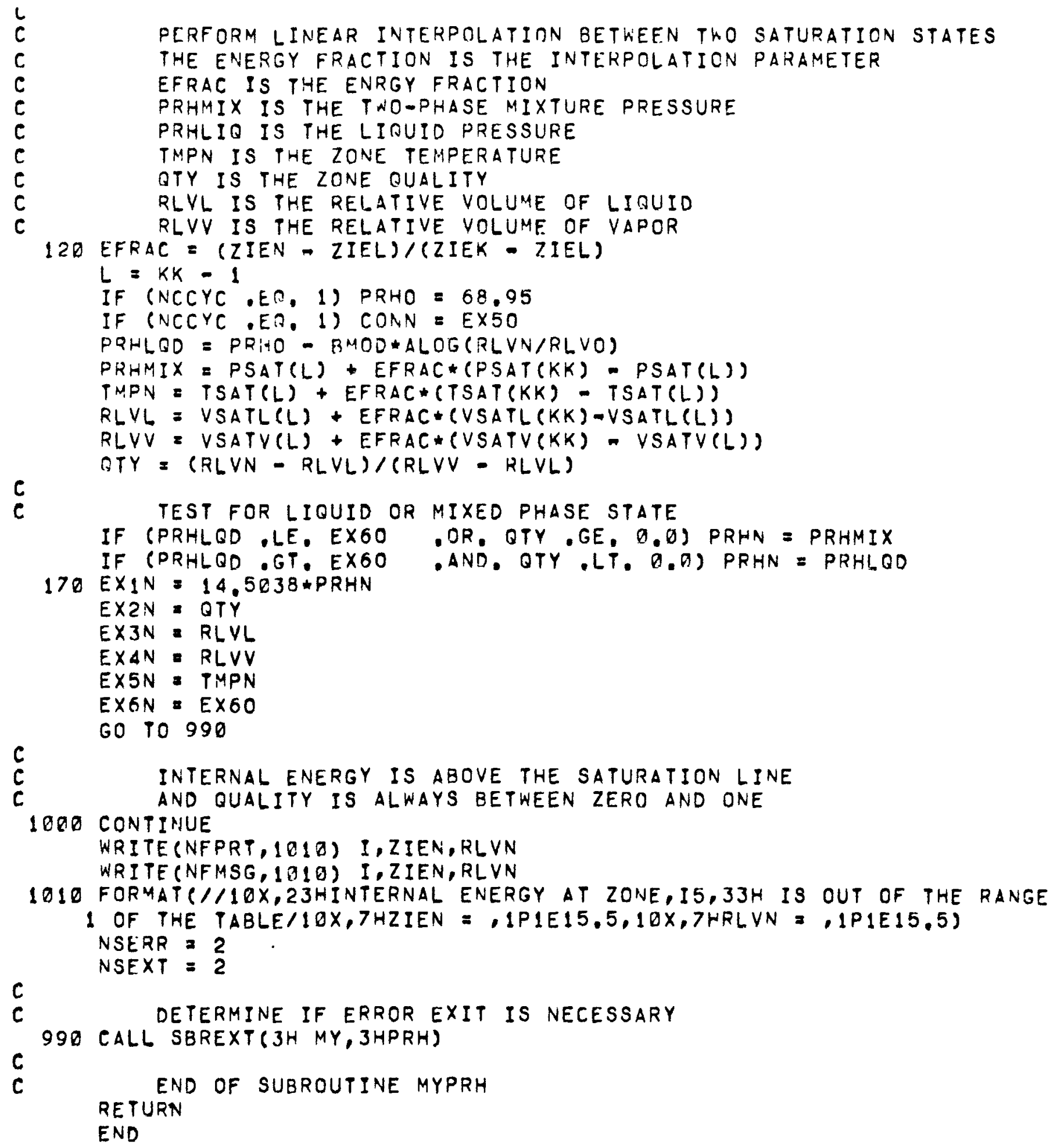




\section{APPENDIX C}

ANALYTICAL SOLUTION OF FLUID FLOW PROBLEMS

Analytical solutions of the differential equation

$$
\frac{\partial \mathbf{v}}{\partial t}=k \frac{\partial^{2} \mathbf{v}}{\partial x^{2}}
$$

can be found in Reference $\mathrm{C}-1$. For solutions to fluid flow problems, the following variable substiutions are made:

$$
\begin{aligned}
& v \equiv \bar{\Phi} \\
& \kappa \equiv \frac{K_{\Phi}}{\rho_{f} g n \beta}
\end{aligned}
$$

and Equation (C.1) becomes

$$
\frac{\partial \Phi}{\partial t}=\frac{{ }^{K} \Phi}{\rho_{f} \operatorname{gn} \beta} \frac{\partial^{2} \Phi}{\partial x^{2}} .
$$

The solution to this equation is given below for three types of fluid flow tests, i.e., for three types of boundary and initial conditions. 


\section{C.1 FLUID FLOW TEST TYPE 1}

For a region defined by $0<\mathrm{x}<\ell$, an initial potential defined by $f(x)$, and the end conditons at constant potentials $\Phi_{1}$ and $\Phi_{2}$, the solution of Equation (C.2) is

$$
\begin{aligned}
\Phi(x, t) & =\Phi_{1}+\left(\Phi_{2}-\Phi_{1}\right) \frac{x}{l} \\
& +\frac{2}{\pi} \sum_{m=1}^{\infty}\left[\frac{\Phi_{a} \cos m \pi-\Phi_{1}}{m} \sin \left(\frac{m \pi x}{l}\right) e^{-\left(\frac{k_{\Phi^{m^{2}} \pi^{2} t}}{\rho_{f} g n \beta l^{2}}\right)}\right] \\
& +\frac{2}{l} \sum_{m=1}^{\infty}\left[\sin \frac{m \pi x}{l} e^{-\left(\frac{k_{\Phi^{2} \pi^{2} t}}{\rho_{f} g n \beta l^{2}}\right)} \int_{0}^{l} f\left(x^{\prime}\right) \sin \frac{m \pi x^{\prime}}{l} d x^{\prime}\right] .
\end{aligned}
$$

\section{C.2 FLUID FLOW TEST TYPE 2}

For the region defined by $0<\mathrm{x}<\ell$, an initial potential of zero, constant fluid flow $Q$ into the medium at $x=l$, and no fluid flow at $x=0$, the solution of Equation (C.2) is

$$
\begin{aligned}
& \Phi(x, t)=\frac{Q t}{\rho_{f}^{g n} \beta l}+\frac{Q l}{K_{\Phi}}\left\{\frac{3 x^{2}-l^{2}}{6 l^{2}}\right. \\
& \left.-\frac{2}{\pi^{2}} \sum_{m=1}^{\infty}\left[\frac{(-1)^{m}}{m^{2}} e^{-\left(\frac{k_{\Phi^{m}}{ }^{2} \pi^{2} t}{\rho_{f} g n \beta l^{2}}\right) \cos \left(\frac{m \pi x}{l}\right)}\right]\right\} \text {. }
\end{aligned}
$$




\title{
C.3 FLUID FLOW TEST TYPE 3
}

For an infinite hollow cylinder with surfaces $r=a$ and $r=b$ kept at constant potentials, $\Phi_{1}$ and $\Phi_{a}$ for $t>0$, and for an initial potential of zero, the solution of Equation (C.2) is

$$
\begin{aligned}
& \Phi(r, t)=-\pi \sum_{m=1}^{\infty}\left\{\left[\frac{\Phi_{2} J_{0}\left(a \alpha_{m}\right)-\Phi_{1} J_{0}\left(b \alpha_{m}\right)}{J_{0}^{2}\left(a \alpha_{m}\right)-J_{0}^{2}\left(b \alpha_{m}\right)}\right] J_{0}\left(a \alpha_{m}\right) U\left(r \alpha_{m}\right)\right. \\
& \left.* e^{-}\left(\frac{k_{\Phi^{m}} \pi^{2} t}{\rho_{f} g n l^{2}}\right)\right\} \\
& +\frac{\Phi_{1} \ln (\mathrm{b} / \mathrm{r})+\Phi_{2} \ln (\mathrm{r} / \mathrm{a})}{\ln (\mathrm{b} / \mathrm{a})}
\end{aligned}
$$

where $J_{0}(r \alpha)$ is a Bessel function of order zero and

$$
\mathrm{U}_{0}(\mathrm{r} \alpha)=\mathrm{J}_{\mathrm{o}}(\mathrm{r} \alpha) \mathrm{Y}_{\mathrm{o}}(\mathrm{b} \alpha)-\mathrm{J}_{\mathrm{o}}(\mathrm{b} \alpha) \mathrm{Y}_{\mathrm{o}}(\mathrm{r} \alpha)
$$

$Y_{0}$ is a Weber's function of order zero and $\alpha$ is a root of $U_{0}(r \alpha)=0$.

\author{
REFERENCES
}

C-1. H. S. Carslaw and J. C. Jaegar, Conductivity of Heat in Solids, 2nd Edition, Oxford University Press, Oxford, 1959. 